Preprint typeset in JHEP style - HYPER VERSION

\title{
Neutrino masses and mixing, lightest neutralino decays and a solution to the $\mu$ problem in supersymmetry
}

\author{
Pradipta Ghosh and Sourov Roy \\ Department of Theoretical Physics and Centre for Theoretical Sciences, \\ Indian Association for the Cultivation of Science, \\ $2 A$ \& $2 B$ Raja S.C. Mullick Road, Kolkata 700 032, India \\ E-mails tppg@iacs.res.in, tpsr@iacs.res.in
}

\begin{abstract}
We examine in detail the neutrino masses and mixing patterns in an extension of the minimal supersymmetric standard model with three gauge-singlet neutrinos and Rparity violation. The Majorana masses for the gauge-singlet neutrinos as well as the usual $\mu$-term for the Higgs superfields are generated at the electroweak scale through the vacuum expectation values of the singlet sneutrinos. The resulting effective mass matrix for the three light neutrinos have contributions from the seesaw mechanism involving the singlet neutrinos as well as due to the mixing with the heavy neutralinos. This model is popularly known in the literature as the " $\mu$ from $\nu$ supersymmetric standard model" ( $\mu \nu \mathrm{SSM})$. We show that even with flavour diagonal neutrino Yukawa couplings, the global data on threeflavour neutrinos can be well accounted for in this scenario, at the tree level. We also analyze the mixing in the chargino and the Higgs sector and calculate the decays of the lightest supersymmetric particle in this model. The decay branching ratios show certain correlations with the neutrino mixing angles, which can be tested at the LHC. Some other phenomenological implications of such a model have been discussed.
\end{abstract}

KEYWORDs: Supersymmetry Phenomenology. 


\section{Contents}

1. Introduction 2

2. The model and its minima 5

2.1 Superpotential 5

2.2 Soft terms 6

2.3 The neutral scalar potential and the electroweak symmetry breaking conditions 6

3. The scalar sector 9

4. The fermionic sector 10

4.1 The neutral fermions 10

4.2 The charged fermions

5. Neutrinos 14

5.1 Seesaw masses 14

5.1.1 Analytical results 14

5.1 .2 Numerical Results 18

5.2 Neutrino mixing 19

5.2.1 Analytical results 20

5.2.2 Numerical Results 22

6. Decays of the lightest neutralino 24

6.1 Correlation between the lightest neutralino decays and neutrino mixing angles 27

6.1 .1 Bino dominated lightest neutralino 28

6.1 .2 Higgsino dominated lightest neutralino 30

$6.1 .3 \nu^{c}$ dominated lightest neutralino 32

7. Summary and Conclusion 37

A. Scalar mass squared matrices 40

A.1 Neutral scalar mass squared matrices 40

A.1.1 CP-odd neutral mass squared matrix 40

A.1.2 CP-even neutral mass squared matrix 41

A.2 Charged scalar mass squared matrix 42

B. Feynman rules 43

B.1 Neutralino-neutralino- $Z^{0}$ and chargino-chargino- $Z^{0}, \gamma$

B.2 Chargino-neutralino - $W^{ \pm}$ 


\section{Introduction}

The experimental results on neutrinos provide strong evidence in favour of non-zero neutrino masses and mixing angles. Various neutrino oscillation experiments suggest that the mixing pattern of the three light neutrinos is bilarge, that is to say there are two large mixing angles and one small mixing angle. The data can be explained very well with the following set of mass squared differences and mixing angles [1, 2]

$$
\begin{aligned}
& 7.05 \times 10^{-5} \mathrm{eV}^{2} \leq \Delta m_{21}^{2} \leq 8.34 \times 10^{-5} \mathrm{eV}^{2} \\
& 2.07 \times 10^{-3} \mathrm{eV}^{2} \leq\left|\Delta m_{31}^{2}\right| \leq 2.75 \times 10^{-3} \mathrm{eV}^{2} \\
& 30^{\circ} \leq \theta_{12} \leq 37^{\circ}, 35^{\circ} \leq \theta_{23} \leq 54^{\circ}, \theta_{13} \leq 13^{\circ}
\end{aligned}
$$

where $\Delta m_{i j}^{2} \equiv m_{i}^{2}-m_{j}^{2}$.

In order to explain the results in eqs.(1.1) - (1.3), one needs to go to a theory beyond the standard model (SM). A very interesting possibility to look for new physics is supersymmetry (SUSY) which has the ability to provide a solution of the so-called 'gauge hierarchy problem' connected with the mass of the Higgs boson. SUSY predicts new particles at the $\mathrm{TeV}$ scale which can be tested at the forthcoming Large Hadron Collider (LHC). Naturally it is tempting to see whether TeV scale SUSY is also one of the candidates which can explain the observed pattern of neutrino mass squared differences and mixing. There have been several proposals in recent times, which attempt to explain the experimental data on neutrinos in the context of a supersymmetric theory. Perhaps the most well studied class of models in this context is the one which includes R-parity violation [3, 4] in the Minimal Supersymmetric Standard Model (MSSM). Neutrino mass squared differences and mixing angles have been calculated under various assumptions and it has been found that the neutrino data can be explained well when contributions to the neutrino mass matrix at the tree and one-loop level are considered [5, 6].

On the other hand, though SUSY provides some elegant solutions to accommodate the experimental data on neutrinos, it has been plagued by a few urgent questions which do not have very satisfactory answers yet. One of them is the so-called " $\mu$-problem" [7] related to the bilinear term $\mu \hat{H}_{1} \hat{H}_{2}$ in the MSSM superpotential. The electroweak symmetry breaking requires the value of $\mu$ to be roughly of the order of a few hundreds of $\mathrm{GeV}$. This requires, in the absence of any fine cancellation, that $\mu$ is roughly of the order of the soft scalar masses and both of them should be somewhere around a $\mathrm{TeV}$ or a few hundreds of $\mathrm{GeV}$. Since $\mu$ parameter respects SUSY, there is no obvious reason why it should be of the same order as SUSY breaking soft scalar masses. This defines the " $\mu$-problem". There have been several attempts to address the solution to this problem and all of them requires the vacuum expectation value(s) $(\mathrm{VEVs})$ of some additional field(s) to generate the $\mu$-term after the symmetry breaking.

One of the solutions is the next-to-minimal supersymmetric standard model (NMSSM), which introduces a superfield $\hat{S}$, singlet under the SM gauge group. The $\mu$ term is absent 
from the superpotential and it arises when the scalar component of $\hat{S}$ acquires a VEV. This VEV is determined in terms of the soft supersymmetry breaking terms through the minimization condition. If the SUSY breaking scale is close to the electroweak (EW) scale then the effective $\mu$ term is also of the order of the EW scale. However, as in the case of MSSM, the NMSSM also cannot explain the observed pattern of neutrino masses and mixing.

It is also important to note in this context, that another very well known mechanism of generating small neutrino masses and bilarge mixing angles in a SUSY model, compatible with the experimental data, is the seesaw mechanism which introduces gauge singlet neutrino superfields. In such cases the MSSM superpotential contains additional terms involving the Yukawa couplings for neutrinos as well as Majorana masses for the gauge singlet neutrinos. In the conventional scenario, the neutrino Yukawa couplings are assumed to be $\sim \mathcal{O}(1)$, whereas the Majorana masses for the gauge singlet neutrinos are taken somewhere around $10^{14} \mathrm{GeV}$ or so. In this case light neutrino masses as small as $10^{-2} \mathrm{eV}$ can be generated. One viable alternative to the usual seesaw mechanism is to consider the $\mathrm{Te} V$-scale seesaw. This possibility is very interesting since it may provide a direct way to probe the gauge singlet neutrino mass scale at the LHC and does not need to introduce a very high energy scale in the theory. However, in order to generate small active neutrino masses one needs to consider neutrino Yukawa couplings to be of order $10^{-6}$. This choice is reasonable since we know that the electron Yukawa coupling should also be of the order of $10^{-6}$.

In this work we study in details, a model of neutrino masses and mixing which introduces the gauge singlet neutrino superfields $\left(\hat{\nu}_{i}^{c}\right)$ to solve the $\mu$ problem in a way similar to that of NMSSM. The terms in the superpotential involving the $\hat{\nu}_{i}^{c}$ include the neutrino Yukawa couplings, the trilinear interaction terms among the singlet neutrino superfields as well as a term which couples the Higgs superfields to the $\hat{\nu}_{i}^{c}$. In addition, there are corresponding soft SUSY breaking terms in the scalar potential. When the scalar components of $\hat{\nu}_{i}^{c}$ get VEVs through the minimization conditions of the scalar potential, an effective $\mu$ term with an EW scale magnitude is generated [8]. In addition, small Majorana masses of the active neutrinos are generated due to the mixing with the neutralinos as well as due to the seesaw mechanism involving the gauge singlet neutrinos. In such a scenario, we aim to explain the experimental data on neutrinos discussed in the beginning. In particular, we show that it is possible to provide a theory of neutrino masses and mixing explaining the experimental data, even with a flavour diagonal neutrino Yukawa coupling matrix, without resort to an arbitrary flavour structure in the neutrino sector. This essentially happens because of the mixing involved in the neutralino-neutrino (both doublet and singlet) system mentioned above. We perform a detailed analytical and numerical work and show that the three flavour neutrino data can be accommodated in such a scenario. In addition, we observe that in this model different neutrino mass hierarchies can also be obtained with correct mixing pattern, at the tree level.

In this model the neutral Higgs bosons mix with the sneutrinos and charged Higgs bosons mix with the charged sleptons. The corresponding scalar, pseudoscalar and charged scalar mass squared matrices are enlarged and we take into account the constraints on the 
parameters coming from the positivity of the squared scalar masses. In the fermionic sector, in addition to the neutralino-neutrino mixing there is mixing also between the charginos and the charged leptons. This can also have implications for phenomenological studies, particularly in the context of future colliders. Because of the mixing between the neutralinos and the neutrinos, the lightest neutralino, which is the lightest superparticle (LSP) for most of the parameter space, can have novel decay modes which can be correlated with the neutrino mixing pattern. This can provide some unique signatures of such a scenario which can be tested at the LHC.

As we have mentioned earlier, that in order to get the correct light neutrino mass scales, the neutrino Yukawa couplings should be of the order of $10^{-6}$ or so. This is because the $\mathrm{TeV}$ scale VEVs of the singlet neutrinos induce Majorana mass terms of themselves also at the $\mathrm{TeV}$ scale. Similarly, the neutralino-neutrino mixing provides correct light neutrino mass scales only when the Yukawa couplings of the neutrinos are somewhere close to that of the electron. This model has been named as the " $\mu \nu \mathrm{SSM}$ " in ref. [8]. Thus in this model, an interesting proposal has been given, where the generation of small neutrino masses and the solution to the $\mu$ problem can be accommodated with the same set of gauge singlet neutrino superfields without introducing an extra singlet such as in the case of NMSSM. The spectrum and parameter space of this model, with three gauge singlet neutrino superfields, were discussed in [9]. However, a detailed discussion of the issue of neutrino masses and bilarge neutrino mixing, in order to accommodate the three flavour neutrino data, has been lacking and that is what we want to provide in the present work. We would like to emphasize here that our analysis shows that even with flavour diagonal neutrino Yukawa couplings, the resulting structure of the effective mass matrix of the light neutrinos can explain the bilarge pattern of mixing. In addition, we explore the correlation between neutrino mixing and the decay pattern of the LSP in this model and discuss some other interesting phenomenological implications.

Other models which address the neutrino experimental data and the $\mu$ problem are essentially extensions of NMSSM. One of these proposals 10 include both the gauge singlet neutrino superfields $\left(\hat{\nu}_{i}^{c}\right)$ and the extra singlet $(\hat{S})$ of the NMSSM. In this case the Majorana masses of the singlet neutrinos are also generated at the EW scale through the VEV of the scalar component of $\hat{S}$. R-parity may be broken spontaneously and the light neutrino masses are generated through the seesaw at the EW scale. Another possibility is discussed in ref.[1]], where the effective bilinear R-parity violating terms are generated through the VEV of the singlet scalar $S$. Naturally, in this case only one neutrino mass is generated at the tree level whereas the other two masses are generated at the loop level. In [12], two neutrino masses are generated at the tree level by including explicit bilinear R-parity violating terms in addition to the R-parity breaking term involving $\hat{S}$.

The plan of the paper is the following. We start with the description of the model in Sec. II by writing down the superpotential and the soft supersymmetry breaking interaction terms. We also derive the minimization equations of the scalar potential and discuss some issues related to the vacuum expectation values of the left sneutrinos. In Sec. III we continue our discussion of the scalar sector with a more detailed look. Sec. IV describes the fermionic sector of the model where neutralinos mix with both the doublet and singlet 
neutrinos and the charginos mix with the charged leptons. In Sec. V we provide a detailed discussion of the effective mass matrix of the three light neutrinos, arising because of the neutralino-neutrino mixing. Analytical expressions of the mass eigenvalues and eigenvectors are derived under certain conditions, using degenerate perturbation theory. We construct the neutrino mixing matrix and show that it is possible to have two large and one small mixing angles in general. A detailed numerical analysis has been performed and we compare our results with that obtained using the approximate analytical formulae. We show that for realistic parameter choices, it is possible to fit the three flavour global neutrino data in this scenario, even with a flavour diagonal neutrino Yukawa coupling matrix. The decays of the lightest supersymmetric particle are discussed in Sec. VI and it has been observed that certain decay branching ratios are correlated with the neutrino mixing angles. We make concluding remarks in Sec. VII with possible future directions of our work. The details of various scalar mass squared matrices and the Feynman rules for the LSP decay calculations are relegated to the appendices.

\section{The model and its minima}

\subsection{Superpotential}

In this section we introduce the model along the lines of ref. [8] , discuss its basic features and set our notations. We introduce three gauge singlet neutrino superfields, $\hat{\nu}_{i}^{c}(i=e, \mu, \tau)$, in addition to the fields of the minimal supersymmetric standard model. The superpotential of the model is written as

$$
\begin{aligned}
W & =\epsilon_{a b}\left(Y_{u}^{i j} \hat{H}_{2}^{b} \hat{Q}_{i}^{a} \hat{u}_{j}^{c}+Y_{d}^{i j} \hat{H}_{1}^{a} \hat{Q}_{i}^{b} \hat{d}_{j}^{c}+Y_{e}^{i j} \hat{H}_{1}^{a} \hat{L}_{i}^{b} \hat{e}_{j}^{c}+Y_{\nu}^{i j} \hat{H}_{2}^{b} \hat{L}_{i}^{a} \hat{\nu}_{j}^{c}\right) \\
& -\epsilon_{a b} \lambda^{i} \hat{\nu}_{i}^{c} \hat{H}_{1}^{a} \hat{H}_{2}^{b}+\frac{1}{3} \kappa^{i j k} \hat{\nu}_{i}^{c} \hat{\nu}_{j}^{c} \hat{\nu}_{k}^{c},
\end{aligned}
$$

where $\hat{H}_{1}$ and $\hat{H}_{2}$ are the down-type and up-type Higgs superfields, respectively. The $\hat{Q}_{i}$ are doublet quark superfields, $\hat{u}_{j}^{c}\left[\hat{d}_{j}^{c}\right]$ are singlet up-type [down-type] quark superfields. The $\hat{L}_{i}$ are the doublet lepton superfields, and the $\hat{e}_{j}^{c}$ are the singlet charged lepton superfields. Here $a, b$ are $\mathrm{SU}(2)$ indices, and $\epsilon_{12}=-\epsilon_{21}=1$. Note that the usual bilinear $\mu$-term of the MSSM is absent from the superpotential whereas two additional trilinear terms are introduced involving the Higgs superfields, $\hat{H}_{1}$ and $\hat{H}_{2}$, and the three gauge singlet neutrino superfields, $\hat{\nu}_{i}^{c}$. This is done by imposing a $Z_{3}$ symmetry which is also used in the case of NMSSM. If the scalar potential of the model is such that non-zero vacuum expectation values of the scalar components $\left(\tilde{\nu}_{i}^{c}\right)$ of the singlet superfields $\hat{\nu}_{i}^{c}$ are induced, an effective bilinear term $\mu \hat{H}_{1}^{a} \hat{H}_{2}^{b}$ is generated, where the coefficient $\mu \equiv \lambda^{i}\left\langle\tilde{\nu}_{i}^{c}\right\rangle$. In the presence of soft supersymmetry breaking, it is usually expected that the VEVs of $\tilde{\nu}_{i}^{c}$ are at the electroweak scale. Hence the value of $\mu$ is of the order of the electroweak scale as long as the dimensionless couplings $\lambda_{i}$ are $\sim \mathcal{O}(1)$. This gives us a solution to the so-called " $\mu$ problem". The last term in the superpotential with the coefficient $\kappa^{i j k}$ is included in order to avoid an unacceptable axion associated to the breaking of a global U(1) symmetry [13]. This term generates effective Majorana masses for the singlet neutrinos at the electroweak scale. 
The last two terms in (2.1) explicitly break lepton number (L) and hence the R-parity, which is defined by $R=(-1)^{L+3 B+2 s}$. Here $\mathrm{B}$ is the baryon number and $\mathrm{s}$ is the spin. Note that $R=+1$ for particles and -1 for superpartners. Since lepton number is explicitely broken, no unwanted massless Majoron appears in this model. One should also notice that the term in the superpotential involving the neutrino Yukawa couplings $Y_{\nu}^{i j}$, generate effective bilinear R-parity violating interactions $\epsilon^{i} \hat{H}_{2} \hat{L}_{i}$. Here $\epsilon^{i}$ is determined in terms of the VEVs of $\tilde{\nu}_{i}^{c}$ and is given by $\epsilon^{i}=Y_{\nu}^{i j}\left\langle\tilde{\nu}_{j}^{c}\right\rangle$. R-parity breaking implies that the lightest supersymmetric particle (LSP) is not stable and it cannot be a candidate for dark matter. The decay of the LSP may produce some interesting signatures at the LHC, which can have certain correlations with the neutrino oscillation data. In addition, one can measure displaced vertices originating from the LSP decay.

It should be mentioned here that neutrino masses and bilarge neutrino mixing have also been studied in an R-parity violating supersymmetric theory with gauge singlet neutrino superfields [14]. However, in that analysis terms of the type $\hat{\nu}^{c} \hat{H}_{1} \hat{H}_{2}$ have been dropped from the superpotential because of very small coefficient. Analysis has also been carried out in the context of the observed baryon asymmetry of the Universe [15]. Finally, one should note that the discrete $Z_{3}$ symmetry of the superpotential is spontaneously broken in the vacuum. This might lead to cosmological domain wall problem [16]. However, the solutions to this problem exist [17] and will also work in this case.

\subsection{Soft terms}

Let us now specify the soft-supersymmetry-breaking terms of this model. We will confine ourselves in the framework of supergravity mediated supersymmetry breaking. The Lagrangian $\mathcal{L}_{\text {soft }}$, containing the soft-supersymmetry-breaking terms is given by

$$
\begin{aligned}
-\mathcal{L}_{\text {soft }} & =\left(m_{\tilde{Q}}^{2}\right)^{i j} \tilde{Q}_{i}^{a^{*}} \tilde{Q}_{j}^{a}+\left(m_{\tilde{u}^{c}}^{2}\right)^{i j} \tilde{u}_{i}^{c^{*}} \tilde{u}_{j}^{c}+\left(m_{\tilde{d}^{c}}^{2}\right)^{i j} \tilde{d}_{i}^{c^{*}} \tilde{d}_{j}^{c}+\left(m_{\tilde{L}}^{2}\right)^{i j} \tilde{L}_{i}^{a^{*}} \tilde{L}_{j}^{a} \\
& +\left(m_{\tilde{e}^{c}}^{2}\right)^{i j} \tilde{e}_{i}^{c^{*}} \tilde{e}_{j}^{c}+m_{H_{1}}^{2} H_{1}^{a^{*}} H_{1}^{a}+m_{H_{2}}^{2} H_{2}^{a^{*}} H_{2}^{a}+\left(m_{\tilde{\nu}^{c}}^{2}\right)^{i j} \tilde{\nu}_{i}^{c^{*}} \tilde{\nu}_{j}^{c} \\
& +\epsilon_{a b}\left[\left(A_{u} Y_{u}\right)^{i j} H_{2}^{b} \tilde{Q}_{i}^{a} \tilde{u}_{j}^{c}+\left(A_{d} Y_{d}\right)^{i j} H_{1}^{a} \tilde{Q}_{i}^{b} \tilde{d}_{j}^{c}+\left(A_{e} Y_{e}\right)^{i j} H_{1}^{a} \tilde{L}_{i}^{b} \tilde{e}_{j}^{c}+\text { H.c. }\right] \\
& +\left[\epsilon_{a b}\left(A_{\nu} Y_{\nu}\right)^{i j} H_{2}^{b} \tilde{L}_{i}^{a} \tilde{\nu}_{j}^{c}-\epsilon_{a b}\left(A_{\lambda} \lambda\right)^{i} \tilde{\nu}_{i}^{c} H_{1}^{a} H_{2}^{b}+\frac{1}{3}\left(A_{\kappa} \kappa\right)^{i j k} \tilde{\nu}_{i}^{c} \tilde{\nu}_{j}^{c} \tilde{\nu}_{k}^{c}+\text { H.c. }\right] \\
& -\frac{1}{2}\left(M_{3} \tilde{\lambda}_{3} \tilde{\lambda}_{3}+M_{2} \tilde{\lambda}_{2} \tilde{\lambda}_{2}+M_{1} \tilde{\lambda}_{1} \tilde{\lambda}_{1}+\text { H.c. }\right) .
\end{aligned}
$$

In eq.(2.2), the first two lines consist of squared-mass terms of squarks, sleptons (including the gauge singlet sneutrinos $\tilde{\nu}_{i}^{c}$ ) and Higgses. The next two lines contain the trilinear scalar couplings. Finally, in the last line of eq.(2.2), $M_{3}, M_{2}$, and $M_{1}$ are Majorana masses corresponding to $S U(3), S U(2)$ and $U(1)$ gauginos $\tilde{\lambda}_{3}, \tilde{\lambda}_{2}$, and $\tilde{\lambda}_{1}$, respectively. The tree-level scalar potential receives the usual $\mathrm{D}$ and $\mathrm{F}$ term contributions, in addition to the terms from $\mathcal{L}_{\text {soft }}$.

\subsection{The neutral scalar potential and the electroweak symmetry breaking con- ditions}

We assume that only the neutral scalar fields develop in general the following vacuum 
expectation values while minimizing the scalar potential:

$$
\left\langle H_{1}^{0}\right\rangle=v_{1}, \quad\left\langle H_{2}^{0}\right\rangle=v_{2}, \quad\left\langle\tilde{\nu}_{i}\right\rangle=\nu_{i}, \quad\left\langle\tilde{\nu}_{i}^{c}\right\rangle=\nu_{i}^{c}
$$

The tree level neutral scalar potential looks like

$$
\begin{aligned}
\left\langle V_{\text {neutral }}\right\rangle & =\left|\sum_{i, j} Y_{\nu}^{i j} \nu_{i} \nu_{j}^{c}-\sum_{i} \lambda^{i} \nu_{i}^{c} v_{1}\right|^{2}+\sum_{j}\left|\sum_{i} Y_{\nu}^{i j} \nu_{i} v_{2}-\lambda^{j} v_{1} v_{2}+\sum_{i, k} \kappa^{i j k} \nu_{i}^{c} \nu_{k}^{c}\right|^{2} \\
& +\left|\sum_{i} \lambda^{i} \nu_{i}^{c} v_{2}\right|^{2}+\sum_{i}\left|\sum_{j} Y_{\nu}^{i j} v_{2} \nu_{j}^{c}\right|^{2}+\left(\frac{g_{1}^{2}+g_{2}^{2}}{8}\right)\left[\sum_{i}\left|\nu_{i}\right|^{2}+\left|v_{1}\right|^{2}-\left|v_{2}\right|^{2}\right]^{2} \\
& +m_{H_{2}}^{2}\left|v_{2}\right|^{2}+m_{H_{1}}^{2}\left|v_{1}\right|^{2}+\sum_{i, j}\left(m_{\tilde{L}}^{2}\right)^{i j} \nu_{i}^{*} \nu_{j}+\sum_{i, j}\left(m_{\tilde{\nu}^{c}}^{2}\right)^{i j} \nu_{i}^{c^{*}} \nu_{j}^{c} \\
& +\left[\sum_{i, j}\left(A_{\nu} Y_{\nu}\right)^{i j} \nu_{i} \nu_{j}^{c} v_{2}-\sum_{i}\left(A_{\lambda} \lambda\right)^{i} \nu_{i}^{c} v_{1} v_{2}+\sum_{i, j, k} \frac{1}{3}\left(A_{\kappa} \kappa\right)^{i j k} \nu_{i}^{c} \nu_{j}^{c} \nu_{k}^{c}+\text { H.c. }\right] .
\end{aligned}
$$

One important thing is to notice that the potential is bounded from below because the coefficient of the fourth power of all the eight superfields are positive. We shall further assume that all the parameters present in the scalar potential are real. From eq.(2.4), the minimization conditions with respect to $\nu_{i}^{c} \nu_{i}, v_{2}, v_{1}$ are

$$
\begin{gathered}
2 \sum_{j} u_{c}^{i j} \zeta^{j}+\sum_{k} Y_{\nu}^{k i} r_{c}^{k} v_{2}^{2}+\rho^{i} \eta+\mu \lambda^{i} v_{2}^{2}+\sum_{j}\left(m_{\tilde{\nu}^{c}}^{2}\right)^{j i} \nu_{j}^{c}+\left(A_{x} x\right)^{i}=0 \\
\sum_{j} Y_{\nu}^{i j} v_{2} \zeta^{j}+\gamma_{g} \xi_{v} \nu_{i}+r_{c}^{i} \eta+\sum_{j}\left(m_{\tilde{L}}^{2}\right)^{j i} \nu_{j}+\sum_{j}\left(A_{\nu} Y_{\nu}\right)^{i j} \nu_{j}^{c} v_{2}=0 \\
\sum_{j} \rho^{j} \zeta^{j}+\sum_{i} r_{c}^{i} v_{2}+\mu^{2} v_{2}-\gamma_{g} \xi_{v} v_{2}+\sum_{i}\left(A_{\nu} Y_{\nu}\right)^{i j} \nu_{i} \nu_{j}^{c}-\left(A_{\lambda} \lambda\right)^{i} \nu_{i}^{c} v_{1}+m_{H_{2}}^{2} v_{2}=0 \\
-\sum_{j} \lambda^{j} v_{2} \zeta^{j}+\gamma_{g} \xi_{v} v_{1}+\mu^{2} v_{1}-\mu \sum_{j} r_{c}^{j} \nu_{j}+m_{H_{1}}^{2} v_{1}-\left(A_{\lambda} \lambda\right)^{i} \nu_{i}^{c} v_{2}=0
\end{gathered}
$$


where

$$
\begin{aligned}
\left(A_{x} x\right)^{i} & =\sum_{j}\left(A_{\nu} Y_{\nu}\right)^{j i} \nu_{j} v_{2}+\sum_{j, k}\left(A_{\kappa} \kappa\right)^{i j k} \nu_{j}^{c} \nu_{k}^{c}-\left(A_{\lambda} \lambda\right)^{i} v_{1} v_{2}, \\
\zeta^{j} & =\sum_{i, k} \kappa^{i j k} \nu_{i}^{c} \nu_{k}^{c}+\sum_{k} Y_{\nu}^{k j} v_{2} \nu_{k}-\lambda^{j} v_{1} v_{2}, \\
\eta & =\sum_{i, j} Y_{\nu}^{i j} \nu_{i} \nu_{j}^{c}-\left(\sum_{i} \lambda^{i} \nu_{i}^{c}\right) v_{1}, \\
\mu & =\sum_{i} \lambda^{i} \nu^{c}{ }_{i}, \\
\gamma_{g} & =\frac{1}{4}\left(g_{1}^{2}+g_{2}^{2}\right), \\
\xi_{v} & =\left(\sum_{i} \nu_{i}^{2}+v_{1}^{2}-v_{2}^{2}\right), \\
\rho^{i} & =\sum_{j}\left(Y_{\nu}^{j i} \nu_{j}-\lambda^{i} v_{1}\right), \\
r_{c}^{i} & =\epsilon^{i}=\sum_{j} Y_{\nu}^{i j} \nu_{j}^{c}, \\
r^{i} & =\sum_{j} Y_{\nu}^{i j} \nu_{j}, \\
u_{c}^{i j} & =\sum_{k} \kappa^{i j k} \nu_{k}^{c} .
\end{aligned}
$$

In deriving the above equations, it has been assumed that $\kappa^{i j k},\left(A_{\kappa} \kappa\right)^{i j k}, Y_{\nu}^{i j},\left(A_{\nu} Y_{\nu}\right)^{i j}$, $\left(m_{\tilde{\nu}^{c}}^{2}\right)^{i j},\left(m_{\tilde{L}}^{2}\right)^{i j}$ are all symmetric in $i, j, k$.

Note that the Dirac masses for neutrinos are given by $m_{D}^{i j} \equiv Y_{\nu}^{i j} v_{2}$. From present day experiments it is well known that neutrino masses are very small. This implies that the neutrino Yukawa couplings must also be very small $\sim \mathcal{O}\left(10^{-7}\right)$, in order to get correct neutrino mass scale using $\mathrm{TeV}$ scale seesaw mechanism. This immediately tells us that in the limit $Y_{\nu}^{i j} \rightarrow 0$, eq. (2.6) implies that $\nu_{i} \rightarrow 0$. So in order to get appropriate neutrino mass scale both $Y_{\nu}^{i j}$ and $\nu_{i}$ have to be small.

Ignoring the terms of the second order in $Y_{\nu}^{i j}$ and assuming $\left(\nu_{i}^{2}+v_{1}^{2}-v_{2}^{2}\right) \approx\left(v_{1}^{2}-v_{2}^{2}\right)$, $\left(m_{\tilde{L}}^{2}\right)^{i j}=\left(m_{\tilde{L}}^{2}\right) \delta^{i j}$, we can easily solve eq.(2.6) as (using eq. (2.9))

$$
\nu_{i} \approx-\left\{\frac{Y_{\nu}^{i k} u_{c}^{k j} v_{2}-\mu v_{1} Y_{\nu}^{i j}+\left(A_{\nu} Y_{\nu}\right)^{i j} v_{2}}{\gamma_{g}\left(v_{1}^{2}-v_{2}^{2}\right)+\left(m_{\tilde{L}}^{2}\right)}\right\} \nu_{j}^{c}+\left\{\frac{Y_{\nu}^{i j} \lambda^{j} v_{1} v_{2}^{2}}{\gamma_{g}\left(v_{1}^{2}-v_{2}^{2}\right)+\left(m_{\tilde{L}}^{2}\right)}\right\} .
$$

Note from eq.(2.10), that the left handed sneutrinos can acquire, in general, non-vanishing, non-degenerate VEVs even in the limit of zero vacuum expectation values of the gauge singlet sneutrinos. However, zero VEVs of all the three gauge singlet sneutrinos is not an acceptable solution since in that case no $\mu$-term will be generated. Moreover, one needs to ensure that the extremum value of the potential corresponds to the minimum of the potential, by studying the second derivatives. 


\section{The scalar sector}

The scalar sector of this model enhances from that of MSSM, because of the choice of the superpotential in eq.(2.1) (fourth, fifth and the sixth term). In this case, the neutral Higgs bosons can mix with both the doublet and gauge-singlet sneutrinos. The CPodd(pseudoscalar) and CP-even(scalar) mass squared matrices are now $8 \times 8$, considering all three generations of doublet and singlet sneutrinos. Similarly the charged Higgs can mix with the charged sleptons and thus the charged scalar mass squared matrix is enhanced to $8 \times 8$. We have considered only the CP-preserving case and hence all the VEVs are chosen to be real. The scalar sector of this model has been addressed also in a recent work [9]. The details of various scalar mass squared matrices are given in appendix A.

For our analytical and numerical calculations in the later part of the paper, we have assumed that $\left(m_{\tilde{L}^{2}}^{2}\right)^{i j}=\left(m_{\tilde{L}^{2}}^{2}\right) \delta^{i j},\left(m_{\tilde{\nu}^{c}}^{2}\right)^{i j}=\left(m_{\tilde{\nu}^{c}}^{2}\right) \delta^{i j}$, and $Y_{\nu}^{i j}=0$, if $i \neq j$. We have further assumed that $\kappa^{i j k}$ are flavour-diagonal as well as flavour-blind, i.e., $\kappa^{i j k}=\kappa$ if $i=j=k$ and zero otherwise. Similarly, we have assumed a flavour-blind coupling $\lambda^{i}=\lambda$ for $i=1,2,3$. We will see that even with such simplifying assumptions, we can fit the global three flavour neutrino data in this model. We will use the following procedure for all our subsequent analysis. Using the minimization conditions, we will solve for the vacuum expectation values $\nu_{i}$ and $\nu_{i}^{c}$. We will choose the parameters in such a way that the values of $\nu_{i}^{c}$ will give an acceptable number for the $\mu$-parameter $\left(\mu=\lambda \sum_{i} \nu_{i}^{c}\right)$. As a cross check we confirm the existence of two Goldstone bosons in the pseudoscalar and charged scalar mass-squared matrices. In addition, we check that all the eigenvalues of the scalar, pseudoscalar, and charged scalar mass-squared matrices (apart from the Goldstone bosons) should come as positive for a minima.

Additional constraints on the parameter space can come from the existence of false minima. A detailed discussion on this issue has been presented in ref. [9] and the regions excluded by the existence of false minima have been shown. One can check from these figures that mostly the lower part of the region allowed by the absence of tachyons, are excluded by the existence of false minima. In our analysis, we have chosen the parameter points in such a way that they should be well above the regions disallowed by the existence of false minima. Nevertheless, in the case of gauge-singlet neutrino $\left(\nu^{c}\right)$ dominated lightest neutralino (to be discussed later), the value of $\kappa$ that we have chosen is 0.07 with two different values of $\lambda$, namely, 0.1 and 0.29 . In this case, there is a possibility that these points might fall into the regions disallowed by the existence of false minima. However, we have checked that even if we take the value of $\kappa$ to be higher ( 0.2 or so), with appropriately chosen $\lambda$, our conclusions do not change much. For such a point in the parameter space, it is likely that the existence of false minima can be avoided. A more detailed study on this issue is beyond the scope of the present paper.

Let us also mention here that the sign of the $\mu$-term is controlled by the sign of the $\mathrm{VEV} \nu^{c}$ (assuming a positive $\lambda$ ), which is cotrolled by the signs of $A_{\lambda} \lambda$ and $A_{\kappa} \kappa$. If $A_{\lambda} \lambda$ is negative and $A_{\kappa} \kappa$ is positive then the sign of the $\mu$ parameter is negative whereas for opposite signs of the above quantities, we get a positive sign for the $\mu$ parameter.

The scalar mass-squared matrices (both CP odd and CP even) and the vacuum ex- 
pectation values $\nu_{i}^{c}$ are not very sensitive to the change in neutrino Yukawa couplings $\left(Y_{\nu}\right.$ $\left.\sim \mathcal{O}\left(10^{-7}\right)\right)$ and the corresponding soft parameter $A_{\nu} Y_{\nu}\left(\sim \mathcal{O}\left(10^{-4}\right) \mathrm{GeV}\right)$. On the other hand, the values of $\tan \beta$ and the coefficients $\lambda$ and $\kappa$ are very important in order to satisfy various constraints on the scalar sector mentioned earlier. In fig.1, we have plotted the allowed regions in the $(\lambda-\kappa)$ plane for $\tan \beta=10$.

The values of other parameters are chosen to be $m_{\tilde{L}}=400 \mathrm{GeV}, m_{\tilde{\nu}^{c}}=300 \mathrm{GeV}$, $Y_{\nu}^{11}=5.0 \times 10^{-7}, Y_{\nu}^{22}=4.0 \times 10^{-7}, Y_{\nu}^{33}=$ $3.0 \times 10^{-7},\left(A_{\nu} Y_{\nu}\right)^{i j}=1 \mathrm{TeV} \times Y_{\nu}^{i j},\left(A_{\lambda} \lambda\right)=$ $-1 \mathrm{TeV} \times \lambda$, and $\left(A_{\kappa} \kappa\right)=1 \mathrm{TeV} \times \kappa$. The upper limit of the value of $\kappa$ is taken to be $\sim 0.7$ because of the constraints coming from the existence of Landau pole $[9]$. With these values of different parameters satisfying the constraints in the scalar sector, we will go on to calculate the neutrino masses and the mixing patterns as well as the decays of the lightest neutralino in this model as discussed in the next few sections.

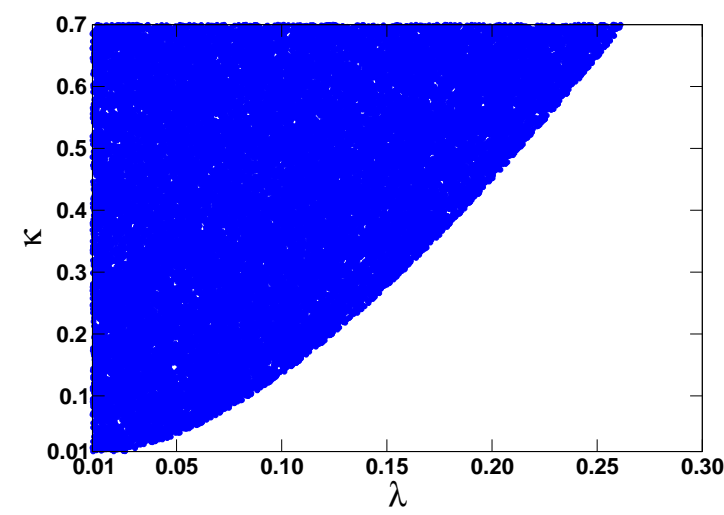

Figure 1: Allowed regions in $(\lambda-\kappa)$ plane which satisfy various constraints on the scalar sector, for $\tan \beta=10$. $\lambda$ and $\kappa$ were allowed to vary from 0.005 to 0.50 and 0.005 to 0.70 , respectively.

It should be mentioned at this point that the radiative corrections to the light Higgs mass, can be significant in some regions of the parameter space as discussed in ref.[9]. It has been shown that the light Higgs mass larger than the LEP lower limit of $114 \mathrm{GeV}$ can be obtained with the value of $A_{t}$ (trilinear coupling in the scalar sector for the stop) within 1-2.4 TeV and when the mixing of the light Higgs with the right-handed sneutrino is small. The latter requirement is fulfilled in most of the cases that we have considered and in some cases the mixing is slightly larger. However, there is always the freedom of choosing the value of $A_{t}$ appropriately. Hence, it would be fair to say that the experimental limits on the light Higgs boson mass can be satisfied in our analysis. The parameter points we have chosen here are sample points with different dominant composition of the lightest neutralino. Since we have a large parameter space, it is always possible to choose a different parameter point with the same characteristic features satisfying all the experimental constraints.

\section{The fermionic sector}

\subsection{The neutral fermions}

In this model, because of the breaking of R-parity, two neutral gauginos, $\tilde{B}^{0}\left(=-i \tilde{\lambda}_{1}\right)$ and $\tilde{W}_{3}^{0}\left(=-i \tilde{\lambda}_{2}^{3}\right)$ and two neutral higgsinos $\left(\tilde{H}_{1}^{0}\right.$ and $\left.\tilde{H}_{2}^{0}\right)$ are now mixed with the neutrinos (both $\nu_{i}$ and $\nu_{i}^{c}$ ). As can be seen from the superpotential (fourth and fifth term in eq. (2.1)), the fermionic partners of $\tilde{\nu}_{i}^{c}$ and $\tilde{\nu}_{i}$ mix with the neutral higgsinos [8, 9]. The neutral gauginos are mixed with the left-handed neutrinos through the vacuum expectation values of the doublet sneutrinos. Mass matrices for the neutral and charged fermion sectors, 
involving all three generations of neutrinos (both doublet and singlet) and charged leptons, have been addressed also in [9].

In the weak interaction basis defined by

$$
\Psi^{0^{T}}=\left(\tilde{B}^{0}, \tilde{W}_{3}^{0}, \tilde{H}_{1}^{0}, \tilde{H}_{2}^{0}, \nu_{e}^{c}, \nu_{\mu}^{c}, \nu_{\tau}^{c}, \nu_{e}, \nu_{\mu}, \nu_{\tau}\right)
$$

the neutral fermion mass term in the Lagrangian is of the form

$$
\mathcal{L}_{\text {neutral }}^{\text {mass }}=-\frac{1}{2} \Psi^{0^{T}} \mathcal{M}_{n} \Psi^{0}+\text { H.c. },
$$

where $\mathcal{M}_{n}$ includes all three generations of doublet and gauge singlet neutrinos and thus it is a $10 \times 10$ matrix. The massless neutrinos become massive due to this mixing with the neutralinos and the gauge singlet neutrinos. The three lightest eigenvalues of this $10 \times 10$ neutralino mass matrix correspond to the three light physical neutrinos and their masses have to be very small in order to satisfy the experimental data on massive neutrinos. The matrix $\mathcal{M}_{n}$ can be written in the following manner

$$
\mathcal{M}_{n}=\left(\begin{array}{cc}
M_{7 \times 7} & m_{3 \times 7}^{T} \\
m_{3 \times 7} & 0_{3 \times 3}
\end{array}\right),
$$

where

$$
M_{7 \times 7}=\left(\begin{array}{ccccccc}
M_{1} & 0 & -\frac{g_{1}}{\sqrt{2}} v_{1} & \frac{g_{1}}{\sqrt{2}} v_{2} & 0 & 0 & 0 \\
0 & M_{2} & \frac{g_{2}}{\sqrt{2}} v_{1} & -\frac{g_{2}}{\sqrt{2}} v_{2} & 0 & 0 & 0 \\
-\frac{g_{1}}{\sqrt{2}} v_{1} & \frac{g_{2}}{\sqrt{2}} v_{1} & 0 & -\mu & -\lambda^{e} v_{2} & -\lambda^{\mu} v_{2} & -\lambda^{\tau} v_{2} \\
\frac{g_{1}}{\sqrt{2}} v_{2} & -\frac{g_{2}}{\sqrt{2}} v_{2} & -\mu & 0 & \rho^{e} & \rho^{\mu} & \rho^{\tau} \\
0 & 0 & -\lambda^{e} v_{2} & \rho^{e} & 2 u_{c}^{e e} & 2 u_{c}^{e \mu} & 2 u_{c}^{e \tau} \\
0 & 0 & -\lambda^{\mu} v_{2} & \rho^{\mu} & 2 u_{c}^{\mu e} & 2 u_{c}^{\mu \mu} & 2 u_{c}^{\mu \tau} \\
0 & 0 & -\lambda^{\tau} v_{2} & \rho^{\tau} & 2 u_{c}^{\tau e} & 2 u_{c}^{\tau \mu} & 2 u_{c}^{\tau \tau}
\end{array}\right),
$$

and

$$
m_{3 \times 7}=\left(\begin{array}{ccccccc}
-\frac{g_{1}}{\sqrt{2}} \nu_{e} & \frac{g_{2}}{\sqrt{2}} \nu_{e} & 0 & r_{c}^{e} & Y_{\nu}^{e e} v_{2} & Y_{\nu}^{e \mu} v_{2} & Y_{\nu}^{e \tau} v_{2} \\
-\frac{g_{1}}{\sqrt{2}} \nu_{\mu} & \frac{g_{2}}{\sqrt{2}} \nu_{\mu} & 0 & r_{c}^{\mu} & Y_{\nu}^{\mu e} v_{2} & Y_{\nu}^{\mu \mu} v_{2} Y_{\nu}^{\mu \tau} v_{2} \\
-\frac{g_{1}}{\sqrt{2}} \nu_{\tau} & \frac{g_{2}}{\sqrt{2}} \nu_{\tau} & 0 & r_{c}^{\tau} & Y_{\nu}^{\tau e} v_{2} & Y_{\nu}^{\tau \mu} v_{2} Y_{\nu}^{\tau \tau} v_{2}
\end{array}\right) .
$$

Note that the top-left $4 \times 4$ block of the matrix $M_{7 \times 7}$ is the usual neutralino mass matrix of the MSSM. The bottom right $3 \times 3$ block is the Majorana mass matrix of gauge singlet neutrinos, which will be taken as diagonal in our subsequent analysis. The entries 
of $M_{7 \times 7}$ are in general of the order of the electroweak scale and the entries of $m_{3 \times 7}$ are much smaller $\left(\sim \mathcal{O}\left(10^{-5} \mathrm{GeV}\right)\right)$. Hence, the matrix (4.3) has a seesaw structure that will give rise to three very light eigenvalues corresponding to three light neutrinos. The correct neutrino mass scale of $\sim 10^{-2} \mathrm{eV}$ can easily be obtained with such a structure of the $10 \times 10$ neutralino mass matrix. In this work our focus would be to see if one can obtain the correct mass-squared differences and the mixing pattern for the light neutrinos even if we consider flavour diagonal neutrino Yukawa couplings in eq.4.5) (i.e. with a diagonal Dirac neutrino mass matrix). This makes the analysis simpler with a reduced number of parameters and makes the model more predictive. As we will show later, it is possible to find out the correct mixing pattern and the mass hierarchies (both normal and inverted) among the light neutrinos in such a situation, at the tree level.

In order to obtain the physical neutralino states, one needs to diagonalize the $10 \times 10$ matrix $\mathcal{M}_{n}$. As in the case of MSSM, the symmetric mass matrix $\mathcal{M}_{n}$ can be diagonalized with one unitary matrix $N$. The mass eigenstates are defined by

$$
\tilde{\chi}_{i}^{0}=N_{i j} \Psi_{j}^{0}, \quad i, j=1, \ldots, 10
$$

where the $10 \times 10$ unitary matrix $N$ satisfies

$$
N^{*} \mathcal{M}_{n} N^{-1}=\mathcal{M}_{D}^{0}
$$

with the diagonal neutralino mass matrix denoted as $\mathcal{M}_{D}^{0}$. The matrix $N$ may be chosen in such a way that elements of $\mathcal{M}_{D}^{0}$ are real and non-negative. In our analysis we will assume that all the entries in the $10 \times 10$ neutralino mass matrix $M_{n}$ are real. Seven eigenstates of this matrix are heavy, i.e. of the order of the electroweak scale. Out of these seven states, there are four states which are usually very similar to the MSSM neutralinos. The remaining three states are mostly dominated by $\nu_{i}^{c}$. It is, in general, very difficult to predict the nature of the lightest of these seven states since that depends on several unknown parameters. In our analysis of the decays of the lightest supersymmetric particle (LSP), we will concentrate on three different possibilities: (i) lightest state is dominated by the bino component, (ii) higgsino dominated lightest state, and (iii) gauge singlet neutrinos $\nu_{i}^{c}$ form the lightest state. The last possibility is very interesting since in this case we have the opportunity to produce the gauge singlet neutrinos at the LHC and study their properties through the R-parity violating decay modes. This way one has a direct probe to the seesaw scale at the LHC.

\subsection{The charged fermions}

In the charged fermion sector, the charged gauginos and charged higgsinos mix with the charged leptons because of the presence of the effective bilinear RPV parameters $\epsilon^{i} \equiv$ $\sum_{j} Y_{\nu}^{i j} \nu_{j}^{c}$ and the sneutrino VEVs $\nu_{i}$. This is similar to the case of MSSM with bilinear RPV with the parameter $\mu$ defined as $\mu=\sum_{i} \lambda^{i} \nu_{i}^{c}$. Since we want to calculate the decays of the lightest neutralino, we also need to know the mass eigenvalues and the mixing matrices in the charged fermion sector. Because of this reason, we discuss the chargino mass matrix 
in some details. In the weak interaction basis defined by

$$
\begin{aligned}
& \Psi^{+T}=\left(-i \tilde{\lambda}_{2}^{+}, \tilde{H}_{2}^{+}, e_{R}^{+}, \mu_{R}^{+}, \tau_{R}^{+}\right), \\
& \Psi^{-T}=\left(-i \tilde{\lambda}_{2}^{-}, \tilde{H}_{1}^{-}, e_{L}^{-}, \mu_{L}^{-}, \tau_{L}^{-}\right) .
\end{aligned}
$$

The charged fermion mass term in the Lagrangian is of the form

$$
\mathcal{L}_{\text {charged }}^{\text {mass }}=-\frac{1}{2}\left(\Psi^{+^{T}} \Psi^{-^{T}}\right)\left(\begin{array}{cc}
0_{5 \times 5} & m_{5 \times 5}^{T} \\
m_{5 \times 5} & 0_{5 \times 5}
\end{array}\right)\left(\begin{array}{c}
\Psi^{+} \\
\Psi^{-}
\end{array}\right) .
$$

Here we have included all three generations of charged leptons and assumed that the charged lepton Yukawa couplings are in the diagonal form. Also, $-i \lambda^{ \pm}$are the twocomponent charged Wino fields and $\tilde{H}_{1}^{-}$and $\tilde{H}_{2}^{+}$are the two-component charged higgsino fields. The matrix $m_{5 \times 5}$ is given by

$$
m_{5 \times 5}=\left(\begin{array}{ccccc}
M_{2} & g_{2} v_{2} & 0 & 0 & 0 \\
g_{2} v_{1} & \mu & -Y_{e}^{e e} \nu_{e}-Y_{e}^{\mu \mu} \nu_{\mu}-Y_{e}^{\tau \tau} \nu_{\tau} \\
g_{2} \nu_{e} & -r_{c}^{e} & Y_{e}^{e e} v_{1} & 0 & 0 \\
g_{2} \nu_{\mu}-r_{c}^{\mu} & 0 & Y_{e}^{\mu \mu} v_{1} & 0 \\
g_{2} \nu_{\tau}-r_{c}^{\tau} & 0 & 0 & Y_{e}^{\tau \tau} v_{1}
\end{array}\right) .
$$

The charged fermion masses are obtained by applying a bi-unitary transformation such that

$$
U^{*} m_{5 \times 5} V^{-1}=\mathcal{M}_{D}^{ \pm}
$$

where $U^{*}$ and $V$ are two unitary matrices and $\mathcal{M}_{D}^{ \pm}$is the diagonal matrix with non-negative entries corresponding to the physical fermion masses. The two-component mass eigenstates are defined by

$$
\begin{aligned}
& \chi_{i}^{+}=V_{i j} \Psi_{j}^{+}, \\
& \chi_{i}^{-}=U_{i j} \Psi_{j}^{-}, \quad i, j=1, \ldots, 5 .
\end{aligned}
$$

Nevertheless, we notice that the 13, 14, and 15 elements of the chargino mass matrix (eq. (4.10) ) are vanishing and given the orders of magnitude of various parameters, we also see that the values of the other off-diagonal entries (except for 12 and 21 elements) are very small. This indicates that the physical charged lepton eigenstates will have a very small admixture of charged higgsino and charged gaugino states. So we can very well assume (also verified numerically) that this mixing has very little effect on the mass eigenstates of the charged leptons. Thus, while writing down the neutrino mixing matrix, it will be justified to assume that one is working in the basis where the charged lepton mass matrix is already in the diagonal form. 


\section{Neutrinos}

\subsection{Seesaw masses}

When lepton-number violation is allowed, the effective light neutrino mass matrix arising via the seesaw mechanism is in general given by

$$
M^{\nu}=-m_{3 \times 7} M_{7 \times 7}^{-1} m_{3 \times 7}^{T} .
$$

As discussed in the previous section, $m_{3 \times 7}$ is the so-called Dirac neutrino mass matrix and $M_{7 \times 7}$ is the matrix for the heavy states and contains $\Delta L=2$ mass terms for right chiral neutrinos. In order to find out the neutrino mass-squared differences and mixing angles, one must diagonalize the matrix $M^{\nu}$ to find out the eigenvalues and the eigenvectors. Before discussing the detailed numerical results, let us try to understand the characteristic features of this neutrino mass matrix analytically. Let us note that the neutrino masssquared differences indicate three possible scenarios for the light neutrino mass spectrum. They are (i) Normal hierarchy corresponding to $m_{1} \approx m_{2} \sim \sqrt{\Delta m_{21}^{2}}, m_{3} \sim \sqrt{\left|\Delta m_{31}^{2}\right|}$, (ii) Inverted hierarchy: $m_{1} \approx m_{2} \sim \sqrt{\left|\Delta m_{31}^{2}\right|}, m_{3} \ll \sqrt{\left|\Delta m_{31}^{2}\right|}$ and (iii) Degenerate masses:

$m_{1} \approx m_{2} \approx m_{3} \gg \sqrt{\left|\Delta m_{31}^{2}\right|}$, where $m_{1}, m_{2}$, and $m_{3}$ are the three light neutrino mass eigenvalues.

\subsubsection{Analytical results}

Let us make a few simplifications in order to diagonalize the effective light neutrino mass matrix. One should note that the neutrino mass matrix involves the vacuum expectation values for the doublet and the gauge singlet sneutrinos. Hence we also make some simplifying assumptions for the parameters appearing in the scalar sector. Some of these assumptions have already been mentioned in the scalar sector but here we repeat them for the convenience of the reader. We have defined

$$
\begin{aligned}
& \kappa^{i j k}=\kappa, \text { if } i=j=k, \text { and zero otherwise, } \\
& \left(A_{\kappa} \kappa\right)^{i j k}=\left(A_{\kappa} \kappa\right), \text { if } i=j=k, \text { and zero otherwise, } \\
& Y_{\nu}^{i j}=0, \text { if } i \neq j, \\
& \left(A_{\nu} Y_{\nu}\right)^{i j}=0, \text { if } i \neq j, \\
& \lambda^{1}=\lambda^{2}=\lambda^{3}=\lambda, \\
& \left(A_{\lambda} \lambda\right)^{1}=\left(A_{\lambda} \lambda\right)^{2}=\left(A_{\lambda} \lambda\right)^{3}=\left(A_{\lambda} \lambda\right), \\
& \left(m_{\tilde{L}^{2}}^{2}\right)^{i j}=\left(m_{\tilde{L}^{2}}^{2}\right) \delta^{i j} \\
& \left(m_{\tilde{\nu}^{c}}^{2}\right)^{i j}=\left(m_{\tilde{\nu}^{c}}^{2}\right) \delta^{i j},
\end{aligned}
$$

where $i, j, k=e, \mu, \tau$ in the flavour basis.

With these assumptions one can solve for $\nu_{i}^{c}$ from the minimization equations for the gauge singlet sneutrinos (eq. (2.5)) and the result is $\nu_{1}^{c}=\nu_{2}^{c}=\nu_{3}^{c}=\nu^{c}$. This can be understood if we neglect the terms proportional to $Y_{\nu}^{2}, Y_{\nu} \nu,\left(A_{\nu} Y_{\nu}\right) \nu$ in the minimization equations and assume that $\lambda, \kappa$ are $\sim \mathcal{O}(1)$ couplings. 
Now let us look at the effective left chiral neutrino mass matrix (eq.(5.1)) in a little more details. Because of the smallness of $Y_{\nu}$ and $\nu_{i}$, one can neglect terms containing $Y_{\nu}^{2} \nu^{2}$ and $Y_{\nu}^{3} \nu$. This way one obtains an approximate analytical expression for the $3 \times 3$ neutrino mass matrix.

$$
\begin{aligned}
& M^{\nu}=\frac{2}{3} \frac{A \nu^{c}}{\Delta}\left(\begin{array}{ccc}
b_{e}^{2} & b_{e} b_{\mu} & b_{e} b_{\tau} \\
b_{e} b_{\mu} & b_{\mu}^{2} & b_{\mu} b_{\tau} \\
b_{e} b_{\tau} & b_{\mu} b_{\tau} & b_{\tau}^{2}
\end{array}\right)+\frac{1}{6 \kappa \nu^{c}}\left(\begin{array}{ccc}
-2 a_{e}^{2} & a_{e} a_{\mu} & a_{e} a_{\tau} \\
a_{e} a_{\mu} & -2 a_{\mu}^{2} & a_{\mu} a_{\tau} \\
a_{e} a_{\tau} & a_{\mu} a_{\tau} & -2 a_{\tau}^{2}
\end{array}\right) \\
& -\frac{2 \lambda \mu}{3 \Delta} \sum_{i} Y_{\nu}^{i i} \nu_{i}\left(v_{2}-\frac{2 \lambda A B}{\Delta}\right)\left(\begin{array}{ccc}
c_{e}^{2} & c_{e} c_{\mu} & c_{e} c_{\tau} \\
c_{e} c_{\mu} & c_{\mu}^{2} & c_{\mu} c_{\tau} \\
c_{e} c_{\tau} & c_{\mu} c_{\tau} & c_{\tau}^{2}
\end{array}\right)
\end{aligned}
$$

where

$$
\begin{aligned}
\Delta & =\lambda^{2}\left(v_{1}^{2}+v_{2}^{2}\right)^{2}+4 \lambda \kappa \nu^{c 2} v_{1} v_{2}-4 M \lambda A \mu, \mu=3 \lambda \nu^{c} \\
A & =\left(\kappa \nu^{c 2}+\lambda v_{1} v_{2}\right) \\
\frac{1}{M} & =\frac{g_{1}^{2}}{M_{1}}+\frac{g_{2}^{2}}{M_{2}} \\
B & =v_{1}\left(v_{1}^{2}+v_{2}^{2}\right)-2 M \mu v_{2} \\
a_{i} & =Y_{\nu}^{i i} v_{2}, b_{i}=\left(Y_{\nu}^{i i} v_{1}+3 \lambda \nu_{i}\right), c_{i}=\nu_{i}
\end{aligned}
$$

with $i, j, k=e, \mu, \tau$.

One can rewrite eq. (5.3) in a compact form as follows

$$
M_{i j}^{\nu}=\frac{2 A \nu^{c}}{3 \Delta} b_{i} b_{j}+\frac{1}{6 \kappa \nu^{c}} a_{i} a_{j}\left(1-3 \delta_{i j}\right)-\frac{2 \lambda \mu}{3 \Delta} \sum_{k} Y_{\nu}^{k k} \nu_{k}\left(v_{2}-\frac{2 \lambda A B}{\Delta}\right) c_{i} c_{j} .
$$

Let us note that the smallness of the left chiral sneutrino VEVs $\left(\nu_{i}<<v_{1}, v_{2}\right)$ allows us to use $m_{Z}^{2} \approx \frac{1}{2}\left(g_{1}^{2}+g_{2}^{2}\right)\left(v_{1}^{2}+v_{2}^{2}\right)$ and $\tan \beta \approx \frac{v_{2}}{v_{1}}$.

The coefficients of the first term in eq. (5.3) (or in eq.(5.5)) is of the order of $\frac{1}{\tilde{m}}$ whereas the coefficient of the second term is $\lesssim \frac{1}{10 \tilde{m}}$, where $\tilde{m}$ is the electroweak (or supersymmetry breaking) scale and we have assumed that the relevant mass scales are at $\tilde{m}$ and $\kappa$ is an order one coupling. The value of the coupling $\lambda$ (determines the value of the $\mu$ parameter), which satisfies the neutrino data as well as the constraints in the scalar sector, is taken to be of the order of $10^{-1}$. On the other hand, the coefficient of the third term is $\sim \frac{1}{\tilde{m}}\left(\frac{\nu_{i}}{\tilde{m}}\right)$. Since $\frac{\nu_{i}}{\tilde{m}} \sim 10^{-6}-10^{-7}$, there is an extra suppression factor in the elements of third term in eq.(5.3), compared to the first two terms. In addition, $b_{i}^{2} \sim a_{i}^{2} \sim c_{i}^{2}$ with a slightly larger value of $a_{i}^{2}$ and $c_{i}^{2}$ compared to $b_{i}^{2}$ in most cases. Hence, one can neglect the third term of eq. (5.3) in comparison to the first two terms. However, in our numerical analysis 
(discussed later) we have kept all the terms in eq. (5.3) and checked that the presence of the third term changes the result in an insignificant manner.

Before going on to find out the expressions for the eigenvalues and the eigenvectors of the effective neutrino mass matrix (eq.(5.3)), let us highlight a few limiting cases which give us some insight regarding the behaviour of the neutrino mass matrix. Neglecting the third term one can rewrite eq.(5.5) in the following manner

$$
\begin{aligned}
M_{i j}^{\nu} \approx \frac{v_{2}^{2}}{6 \kappa \nu^{c}} Y_{\nu}^{i i} Y_{\nu}^{j j}(1-3 \delta i j)- & \frac{1}{2 M}\left[\nu_{i} \nu_{j}+\frac{v_{1} \nu^{c}\left(Y_{\nu}^{i i} \nu_{j}+Y_{\nu}^{j j} \nu_{i}\right)}{\mu}+\frac{Y_{\nu}^{i i} Y_{\nu}^{j j} v_{1}^{2} \nu^{c 2}}{\mu^{2}}\right] \\
\times & {\left[1-\frac{v^{2}}{2 M A \mu}\left(\kappa \nu^{c 2} \sin 2 \beta+\frac{\lambda v^{2}}{2}\right)\right]^{-1} . }
\end{aligned}
$$

Here we have used $v_{2}=v \sin \beta, v_{1}=v \cos \beta$, and $\mu=3 \lambda \nu^{c}$.

In the limit $\nu^{c} \rightarrow \infty$ and $v \rightarrow 0$, eq. (5.6) reduces to

$$
M_{i j}^{\nu} \approx-\frac{\nu_{i} \nu_{j}}{2 M}
$$

which is the first part of the second term of eq.(5.6). In this case the elements of the neutrino mass matrix are bilinears in the left-handed sneutrino VEVs and they appear due to a seesaw effect involving the gauginos. This is called the "gaugino seesaw" effect and neutrino mass generation through this effect is a characteristic feature of the bilinear $R$-parity violating model. This effect is present in this model because we have seen earlier that the effective bilinear R-parity violating terms are generated in the scalar potential as well as in the superpotential through the vacuum expectation values of the gauge singlet sneutrinos. Note that the gaugino seesaw effect can generate mass for only one doublet neutrino.

In the limit $M \rightarrow \infty$, eq. 5.6 reduces to

$$
M_{i j}^{\nu} \approx \frac{v_{2}^{2}}{6 \kappa \nu^{c}} Y_{\nu}^{i i} Y_{\nu}^{j j}(1-3 \delta i j)
$$

which corresponds to the ordinary seesaw effect between the left handed and gauge singlet neutrinos. Remember that the effective Majorana masses for the gauge singlet neutrinos are given by $M_{R}=2 \kappa \nu^{c}$. The ordinary seesaw effect can generate, in general, masses for all the three neutrinos. Thus depending on the magnitudes and the hierarchies of various diagonal neutrino Yukawa couplings $Y_{\nu}^{i i}$, one can generate normal or inverted hierarchy of neutrino masses (combining with the "gaugino seesaw" effect) corresponding to atmospheric and solar mass squared differences, as discussed earlier. In this model it is difficult to obtain a degenerate neutrino spectrum and we do not consider this possibility in our subsequent analysis.

Now let us try to find out the approximate analytical expressions for the eigenvalues and eigenvectors of the effective light neutrino mass matrix using perturbation theory. 
Neglecting the third term in eq.(5.3), the neutrino mass matrix looks like

$$
M^{\nu}=\mathcal{B}\left(\begin{array}{ccc}
b_{e}^{2} & b_{e} b_{\mu} & b_{e} b_{\tau} \\
b_{e} b_{\mu} & b_{\mu}^{2} & b_{\mu} b_{\tau} \\
b_{e} b_{\tau} & b_{\mu} b_{\tau} & b_{\tau}^{2}
\end{array}\right)+\mathcal{A}\left(\begin{array}{ccc}
-2 a_{e}^{2} & a_{e} a_{\mu} & a_{e} a_{\tau} \\
a_{e} a_{\mu} & -2 a_{\mu}^{2} & a_{\mu} a_{\tau} \\
a_{e} a_{\tau} & a_{\mu} a_{\tau} & -2 a_{\tau}^{2}
\end{array}\right),
$$

where $\mathcal{A}=\frac{1}{6 \kappa \nu^{c}}$ and $\mathcal{B}=\frac{2}{3} \frac{A \nu^{c}}{\Delta}$. As we have argued above, the first matrix in eq.(5.9) can be considered as the unperturbed one and the second matrix can be treated as a perturbation over the first one because of the presence of the smaller coefficient $\mathcal{A}$. The eigenvalues of the unperturbed matrix are $\left(0,0, \mathcal{B}\left(b_{e}^{2}+b_{\mu}^{2}+b_{\tau}^{2}\right)\right)$ and the corresponding eigenvectors are $\left(\begin{array}{lll}-\frac{b_{\tau}}{b_{e}} & 0 & 1\end{array}\right)^{T},\left(\begin{array}{lll}-\frac{b_{\mu}}{b_{e}} & 1 & 0\end{array}\right)^{T},\left(\begin{array}{lll}\frac{b_{e}}{b_{\tau}} & \frac{b_{\mu}}{b_{\tau}} & 1\end{array}\right)^{T}$. With the order of magnitudes of various parameters discussed above, the only non-zero eigenvalue determines the atmospheric neutrino mass scale corresponding to the normal hierarchical mass pattern for neutrinos. In order to generate the solar neutrino mass scale one must turn on the perturbation. In this case one should use the unperturbed eigenvectors to get the corrections to the eigenvalues due to perturbation. However, since two of the eigenvalues are zero, one needs to apply the degenerate perturbation theory to evaluate the correction to the eigenvalues. To do this, first we construct a complete set of orthonormal eigenvectors using Gram-Schmidt orthogonalization procedure. The set of orthonormal eigenvectors obtained in this case are

$$
\begin{aligned}
& \mathrm{y}_{1}=\frac{b_{e}}{\sqrt{b_{e}^{2}+b_{\tau}^{2}}}\left(\begin{array}{c}
-\frac{b_{\tau}}{b_{e}} \\
0 \\
1
\end{array}\right), \\
& \mathrm{y}_{2}=\frac{\sqrt{b_{e}^{2}+b_{\tau}^{2}}}{\Omega_{b}}\left(\begin{array}{c}
-\frac{b_{e} b_{\mu}}{b_{e}^{2}+b_{\tau}^{2}} \\
1 \\
-\frac{b_{\mu} b_{\tau}}{b_{e}^{2}+b_{\tau}^{2}}
\end{array}\right), \\
& \mathrm{y}_{3}=\frac{b_{\tau}}{\Omega_{b}}\left(\begin{array}{c}
\frac{b_{e}}{b_{\tau}} \\
\frac{b_{\mu}}{b_{\tau}} \\
1
\end{array}\right),
\end{aligned}
$$

where

$$
\Omega_{b}=\sqrt{b_{e}^{2}+b_{\mu}^{2}+b_{\tau}^{2}}
$$

Using degenerate perturbation theory for this set of orthonormal eigenvectors, the modified eigenvalues $m_{ \pm}^{\prime}$ and $m_{3}^{\prime}$ are obtained as

$$
\begin{aligned}
& m_{ \pm}^{\prime}=-\frac{\mathcal{A}}{\Omega_{b}^{2}}\left\{\Pi_{a b} \pm \sqrt{\left[-3 \Omega_{b}^{2}\left(\Sigma_{a b}\right)^{2}+\left(\Pi_{a b}\right)^{2}\right]}\right\}, \\
& m_{3}^{\prime}=\mathcal{B} \Omega_{b}^{2}-\frac{2 \mathcal{A}}{\Omega_{b}^{2}}\left\{\left(\sum_{i} a_{i} b_{i}\right)^{2}-3 \Lambda_{a b}\right\},
\end{aligned}
$$


where

$$
\begin{aligned}
\Lambda_{a b} & =\sum_{i<j} a_{i} a_{j} b_{i} b_{j}, \\
\Pi_{a b} & =\sum_{i<j}\left(a_{i} b_{j}+a_{j} b_{i}\right)^{2}-\Lambda_{a b}, \\
\Sigma_{a b} & =\sum_{i \neq j \neq k} a_{i} a_{j} b_{k} .
\end{aligned}
$$

As one can see from eq.(5.12), the corrections to the eigenvalues are proportional to the coefficient $\mathcal{A}$ appearing in eq.(5.9). This is the effect of the ordinary seesaw. Let us note in passing that this effect is absent if only one generation of left chiral neutrino is considered, whereas for two and three generations of left chiral neutrino the ordinary seesaw effect exists. This can be understood from the most general calculation involving n-generations of left chiral neutrinos, where the coefficients of $\mathcal{A}$ pick up an extra factor $(n-1)$.

\subsubsection{Numerical Results}

In order to get some idea about the numbers of the mass eigenvalues and to make comparisons between the full numerical results and the results using approximate analytical expression, we look at a sample point in the parameter space. As mentioned earlier, we are considering only the normal hierarchical pattern of neutrino masses. The set of parameters are $M_{1}=325 \mathrm{GeV}, M_{2}=650 \mathrm{GeV}, \lambda=0.06, \kappa=0.65, A_{\lambda} \lambda\left(-A_{\kappa} \kappa\right)=$ $-1 \mathrm{TeV} \times \lambda(\kappa)$ and $\tan \beta=10$.

The choices of diagonal neutrino Yukawa couplings $\left(Y_{\nu}^{i i}\right)$ and corresponding soft parameters $\left(A_{\nu} Y_{\nu}\right)^{i i}$ are very crucial and we take, for this particular calculation, $Y_{\nu}^{e e}=4.57 \times 10^{-7}$, $Y_{\nu}^{\mu \mu}=6.37 \times 10^{-7}, Y_{\nu}^{\tau \tau}=1.80 \times 10^{-7},\left(A_{\nu} Y_{\nu}\right)^{e e}=1.57 \times 10^{-4} \mathrm{GeV},\left(A_{\nu} Y_{\nu}\right)^{\mu \mu}=4.70 \times 10^{-4}$ $\mathrm{GeV}, \quad\left(A_{\nu} Y_{\nu}\right)^{\tau \tau}=3.95 \times 10^{-4} \mathrm{GeV}$. Soft masses of left handed and right handed sleptons are chosen to be $400 \mathrm{GeV}$ and $300 \mathrm{GeV}$, respectively. Later on we will show the allowed regions in the $Y_{\nu}$ planes which satisfy the experimental data on neutrino masses and mixing. For these choices of various parameters, the derived left-handed sneutrino VEVs are $\nu_{e} \sim 10^{-5} \mathrm{GeV}, \nu_{\mu}=1.515 \times 10^{-4} \mathrm{GeV}, \nu_{\tau}=2.133 \times 10^{-4} \mathrm{GeV}$ and right-handed sneutrino VEVs are $\nu^{c}=-588.74 \mathrm{GeV}$. With this set of values the masses of three neutrinos have been found out by direct diagonalization of the matrix obtained using (5.1) and also from the approximate analytical expression using (5.12). It has been observed that even with several simple assumptions (eq.(5.2)), all three generations of left chiral neutrinos acquire non-vanishing, non-degenerate masses at the tree-level. The comparison of the results as obtained from (5.1) and from (5.12) are given in Table 1. One can see that these values are within the $3 \sigma$ limits shown in eqs.(1.1)-(1.2). However, it should be mentioned that if $\lambda, \kappa$ are much less than $\sim \mathcal{O}(1)$ and $Y_{\nu}$ s are much larger than the ones we have considered above, the approximate analytical expression does not produce the correct results for the eigenvalues and the eigenvectors. Obviously, when the neutrino Yukawa couplings are larger one cannot consider the second term in eq.(5.3) as a perturbation to the first term. In our numerical analysis for obtaining the allowed region of parameter space which satisfy 


\begin{tabular}{|c|c|c|c|c|c|}
\hline & \multicolumn{3}{|c|}{$m_{\nu}(\mathrm{eV})\left(\times 10^{3}\right)$} & \multirow{2}{*}{$\begin{array}{c}\Delta m_{21}^{2}\left(\mathrm{eV}^{2}\right) \\
\left(\times 10^{5}\right)\end{array}$} & \multirow{2}{*}{$\begin{array}{c}\Delta m_{31}^{2}\left(\mathrm{eV}^{2}\right) \\
\left(\times 10^{3}\right)\end{array}$} \\
\hline & $m_{1}$ & $m_{2}$ & $m_{3}$ & & \\
\hline eq.(5.1) & 9.970 & 4.169 & 48.23 & 8.203 & 2.307 \\
\hline eq.(5.12) & 9.468 & 4.168 & 47.71 & 7.228 & 2.187 \\
\hline
\end{tabular}

Table 1: Absolute values of the neutrino masses and the mass-squared differences for a sample point of the parameter space discussed in the text. Results for full numerical analysis have been obtained using eq.(5.1). Approximate analytical expressions of eq.5.12) have been used for comparison.

the neutrino data, we have done a full numerical analysis without using the approximate formula.

The numerical values of the solar and atmospheric mass squared differences $\Delta m_{21}^{2}$ and $\Delta m_{31}^{2}$ have also been shown in Table 1 and the results show good agreement. The numerical calculations have been performed with the help of a code developed by us using Mathematica [18]. In our numerical calculations, we have taken for (i) normal hierarchy: $\left.m_{2}\right|_{\max }<1.0 \times 10^{-11} \mathrm{GeV}$ and (ii) inverted hierarchy: $\left.m_{3}\right|_{\max }<1.0 \times 10^{-11} \mathrm{GeV}$.

\subsection{Neutrino mixing}

The left chiral light neutrinos form a $3 \times 3$ mass matrix in the flavour basis. The unitary matrix which diagonalizes this mass matrix can be parameterized as follows [19, provided that the charged lepton mass matrix is already in the diagonal form

$$
\mathcal{U}^{\nu}=\left(\begin{array}{ccc}
c_{12} c_{13} & s_{12} c_{13} & s_{13} e^{-i \delta} \\
-s_{12} c_{23}-c_{12} s_{23} s_{13} e^{-i \delta} & c_{12} c_{23}-s_{12} s_{23} s_{13} e^{-i \delta} & s_{23} c_{13} \\
s_{12} s_{23}-c_{12} c_{23} s_{13} e^{-i \delta} & -c_{12} s_{23}-s_{12} c_{23} s_{13} e^{-i \delta} & c_{23} c_{13}
\end{array}\right)
$$

where $c_{i j}=\cos \theta_{i j}, s_{i j}=\sin \theta_{i j}$, and $i, j$ runs from 1 to 3 . Various neutrino oscillation experiments indicate that $\theta_{12} \approx 34^{\circ}, \theta_{23} \approx 45^{\circ}$, and $\theta_{13} \leq 13^{\circ}$ [20, 21]. This pattern is known as bilarge mixing. In order to understand the consequences of such mixing in the zeroth order, one can approximately take $\theta_{23}=45^{\circ}, \sin \theta_{12}=\frac{1}{\sqrt{3}}$ and $\theta_{13} \approx 0^{\circ}$, something known as tribimaximal structure [22]. Then the unitary matrix turns out to be

$$
\mathcal{U}_{3 \times 3}^{\nu}=\left(\begin{array}{ccc}
\sqrt{\frac{2}{3}} & \frac{1}{\sqrt{3}} & 0 \\
-\frac{1}{\sqrt{6}} & \frac{1}{\sqrt{3}} & \frac{1}{\sqrt{2}} \\
\frac{1}{\sqrt{6}} & -\frac{1}{\sqrt{3}} & \frac{1}{\sqrt{2}}
\end{array}\right)
$$

Given the three mass eigenvalues $m_{1}, m_{2}, m_{3}$, it is possible to use the matrix $\mathcal{U}^{\nu}$ to obtain the mass matrix in the flavour basis as follows, 


$$
\mathcal{U}^{\nu^{-1}} M^{\nu} \mathcal{U}^{\nu}=M_{\text {diag }}^{\nu}
$$

where

$$
M_{\text {diag }}^{\nu}=\left(\begin{array}{ccc}
m_{1} & 0 & 0 \\
0 & m_{2} & 0 \\
0 & 0 & m_{3}
\end{array}\right) \text {. }
$$

We will numerically diagonalize the neutrino mass matrix $M_{\nu}$ obtained in eq.(5.1) and also use the approximate analytical method to find out the neutrino mixing matrix $U^{\nu}$. We will also compare the results obtained using these two methods. However, when we will scan the parameter space to find out the allowed regions where the neutrino experimental data are satisfied, we shall use the full numerical procedure. The advantage of having the approximate analytical expression is that it can give us some insight regarding the conditions on the model parameters for which the bilarge mixing is obtained. We can verify these predictions numerically in some regions of the parameter space. We will try to find out the regions in the models parameters where the numbers in eqs.(1.1)-(1.3) are reproduced.

\subsubsection{Analytical results}

One can construct the neutrino mixing matrix analytically using the degenerate perturbation theory. With the set of orthonormal eigenvectors in eq. (5.10) and the eigenvalues in eq.(5.12), it is possible to write down the eigenvectors of (5.9) in the following form

$$
\begin{gathered}
\left(\mathcal{Y}_{1}\right)_{3 \times 1}=\alpha_{1} y_{1}+\alpha_{2} y_{2}, \\
\left(\mathcal{Y}_{2}\right)_{3 \times 1}=\alpha^{\prime}{ }_{1} y_{1}+\alpha^{\prime}{ }_{2} y_{2}, \\
\left(\mathcal{Y}_{3}\right)_{3 \times 1}=y_{3},
\end{gathered}
$$

where $\alpha_{1}, \alpha_{2}, \alpha_{1}^{\prime}, \alpha_{2}^{\prime}$, are calculated using degenerate perturbation theory and their analytical expressions are given by

$$
\begin{aligned}
& \alpha_{1}= \pm\left(\frac{h_{12}}{\sqrt{h_{12}^{2}+\left(h_{11}-m_{+}^{\prime}\right)^{2}}}\right), \\
& \alpha_{2}=\mp\left(\frac{h_{11}-m_{+}^{\prime}}{\sqrt{h_{12}^{2}+\left(h_{11}-m_{+}^{\prime}\right)^{2}}}\right), \\
& \alpha_{1}^{\prime}= \pm\left(\frac{h_{12}}{\sqrt{h_{12}^{2}+\left(h_{11}-m_{-}^{\prime}\right)^{2}}}\right),
\end{aligned}
$$




$$
\alpha_{2}^{\prime}=\mp\left(\frac{h_{11}-m_{-}^{\prime}}{\sqrt{h_{12}^{2}+\left(h_{11}-m_{-}^{\prime}\right)^{2}}}\right) .
$$

Here $m_{+}^{\prime}, m_{-}^{\prime}$ are given by eq. (5.12) and $h_{11}, h_{12}$ are given by

$$
h_{11}=-\frac{2 \mathcal{A}\left(a_{\tau}^{2} b_{e}^{2}+a_{e} a_{\tau} b_{e} b_{\tau}+a_{e}^{2} b_{\tau}^{2}\right)}{b_{+}^{2}},
$$

and

$$
h_{12}=\frac{\mathcal{A}\left[a_{\mu}\left(a_{\tau} b_{e}-a_{e} b_{\tau}\right) b_{+}^{2}-b_{\mu}\left(2 b_{e} b_{\tau} a_{-}^{2}+a_{e} a_{\tau} b_{-}^{2}\right)\right]}{\Omega_{b} b_{+}^{2}}
$$

where

$$
\begin{aligned}
b_{ \pm}^{2} & =\left(b_{e}^{2} \pm b_{\tau}^{2}\right), \\
a_{-}^{2} & =\left(a_{e}^{2}-a_{\tau}^{2}\right),
\end{aligned}
$$

and $\Omega_{b}$ has been defined in eq.(5.11).

The neutrino mixing matrix $U^{\nu}$ can be constructed using these eigenvectors in eqs.(5.18)(5.20) and it looks like

$$
U^{\nu}=\left(\begin{array}{lll}
\mathcal{Y}_{1} & \mathcal{Y}_{2} & \mathcal{Y}_{3}
\end{array}\right)_{3 \times 3}
$$

Looking at the expressions for the eigenvectors, one can immediately draw a few conclusions regarding the behaviours of the neutrino mixing angles with the model parameters. For example, the (13) mixing angle $\theta_{13}$ is given by

$$
\sin ^{2} \theta_{13}=\frac{b_{e}^{2}}{b_{e}^{2}+b_{\mu}^{2}+b_{\tau}^{2}} .
$$

If we want the (13) mixing angle to be small then one must have $b_{e}^{2} \ll\left(b_{\mu}^{2}+b_{\tau}^{2}\right)$. On the other hand, the (23) mixing angle $\theta_{23}$ is given by

$$
\sin ^{2} \theta_{23}=\frac{b_{\mu}^{2}}{b_{\mu}^{2}+b_{\tau}^{2}}
$$

So, if the (23) mixing is maximal then one would expect $b_{\mu}^{2}=b_{\tau}^{2}$. The solar mixing angle $\theta_{12}$ is approximately given by

$$
\sin ^{2} \theta_{12} \approx 1-\left(\alpha_{1}^{\prime}+\alpha_{2}^{\prime} \frac{b_{e}}{b_{\tau}}\right)^{2}
$$

where $\alpha_{1}^{\prime}$ and $\alpha_{2}^{\prime}$ are given by eqs.(5.23) and (5.24), respectively. In order to have $\theta_{12} \sim$ $35^{\circ}$, the square root of the second term on the right hand side of eq.(5.31) should be approximately 0.8 . In the next sub-section we discuss the patterns of neutrino mixing in this model numerically, and show the allowed regions of the parameter space where the neutrino experimental data are satisfied. 


\subsubsection{Numerical Results}

Let us first calculate the neutrino mixing angles for the parameter point discussed in Table 1. As we have discussed earlier, this parameter point generates the normal hierarchical pattern of neutrino masses. In Table 2, the three mixing angles are shown and they have been evaluated using the direct numerical calculation in eq.(5.1) as well as using the approximate analytical expressions in eq.(5.28). We want to emphasize once again that the approximate formulae have been used just to get some idea about the behaviours of the neutrino masses and mixing angles with various model parame-

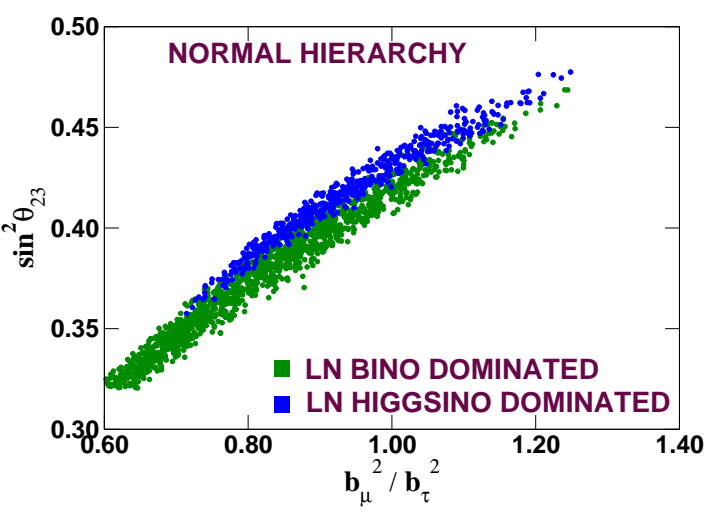

Figure 2: Scatter plot of the neutrino mixing angle $\sin ^{2} \theta_{23}$ as a function of the ratio $\frac{b_{\mu}^{2}}{b_{\tau}^{2}}$. Values of other parameters are described in the text. The lightest neutralino $(\mathrm{LN})$ is either bino or higgsino dominated. ters. This way we can identify the relevant parameters which crucially control the neutrino masses and mixing angles in different regions of the parameter space. However, these formulae are not valid everywhere in the allowed parameter space and in all the plots shown in this paper we have used full numerical calculation using eq.(5.1).

\begin{tabular}{|c|c|c|}
\hline mixing angles in degree & Using (5.1) & Using (5.28) \\
\hline$\theta_{12}$ & 36.438 & 37.287 \\
\hline$\theta_{13}$ & 9.424 & 6.428 \\
\hline$\theta_{23}$ & 38.217 & 42.675 \\
\hline
\end{tabular}

Table 2: Neutrino mixing angles for a sample parameter point discussed in Table 1. Results are shown using eq.(5.1) and eq.(5.28).

We have taken suitable values for $\lambda$ and $\kappa$ in such a way that they fall in the region allowed by the constraints in the scalar sector (similar to the region shown in fig. 1). We can see that for this choice of the parameter space, numerical and approximate analytical results give quite good agreement. Naturally, one would be interested to check the predictions made in eqs. (5.29), (5.30), and (5.31) over a wide region in the parameter space and see the deviations from the full numerical calculations. This has been shown in fig. 2, where we have plotted the value of $\sin ^{2} \theta_{23}$ as a function of the ratio $b_{\mu}^{2} / b_{\tau}^{2}$.

We can see from this figure that for $b_{\mu}^{2}=b_{\tau}^{2}$, the value of $\sin ^{2} \theta_{23}$ varies in the range 0.41 - 0.44, which corresponds to $\theta_{23}$ between $40^{\circ}$ and $42^{\circ}$. On the other hand, eq.(5.30) tells that for $b_{\mu}^{2}=b_{\tau}^{2}, \sin ^{2} \theta_{23}=0.5$. So we see that in this case the result from the numerical calculation is reasonably close to the prediction from the approximate analytical formula. The choices for various parameters are given below. For the gaugino mass parameters $M_{1}$ and $M_{2}$, we take two different sets of values which give us either a bino dominated lightest neutralino or a higgsino dominated lightest neutralino. In order to have a bino dominated lightest neutralino, our choices are $M_{1}=110 \mathrm{GeV}, M_{2}=220 \mathrm{GeV}$, and for 
a higgsino dominated lightest neutralino we take $M_{1}=325 \mathrm{GeV}, M_{2}=650 \mathrm{GeV}$. The same choices will be made for the gaugino mass parameters when we discuss the decays of the lightest neutralino in Sec. VI. Our choice of the ratio of the gaugino mass parameters at the electroweak scale is motivated by the assumption of universal gaugino mass at the grand unified theory scale. The value of $\kappa$ is taken as 0.65 which satisfies the constraints from the scalar sector. We have taken two different values of $\lambda$ corresponding to a bino or a higgsino dominated lightest neutralino. For the bino dominated case $\lambda=0.13$, and for the higgsino dominated case $\lambda=0.06$. The corresponding values for $A_{\lambda} \lambda=-\lambda \times 1 \mathrm{TeV}$ and $A_{\kappa} \kappa=\kappa \times 1 \mathrm{TeV}$. The three diagonal neutrino Yukawa couplings $\left(Y_{\nu}^{i i}\right)$ vary randomly in different ranges

$$
\begin{aligned}
& 3.55 \times 10^{-7} \leq Y_{\nu}^{11} \leq 5.45 \times 10^{-7} \\
& 5.55 \times 10^{-7} \leq Y_{\nu}^{22} \leq 6.65 \times 10^{-7} \\
& 1.45 \times 10^{-7} \leq Y_{\nu}^{33} \leq 3.35 \times 10^{-7}
\end{aligned}
$$

The corresponding soft parameters $\left(A_{\nu}^{i i}\right)$ also vary randomly in different ranges such that the parameters $\left(A_{\nu} Y_{\nu}\right)^{i i}$ effectively vary as follows

$$
\begin{aligned}
& 1.25 \times 10^{-4} \leq\left(A_{\nu} Y_{\nu}\right)^{11} \leq 1.95 \times 10^{-4} \\
& 3.45 \times 10^{-4} \leq\left(A_{\nu} Y_{\nu}\right)^{22} \leq 4.95 \times 10^{-4} \\
& 2.35 \times 10^{-4} \leq\left(A_{\nu} Y_{\nu}\right)^{33} \leq 4.20 \times 10^{-4}
\end{aligned}
$$

The allowed regions in the $\lambda-\kappa$ plane are not very sensitive to the values of $Y_{\nu}$ and $A_{\nu} Y_{\nu}$ due to their smallness. Hence we choose them different for different cases, in order to accommodate the three flavour global neutrino data.

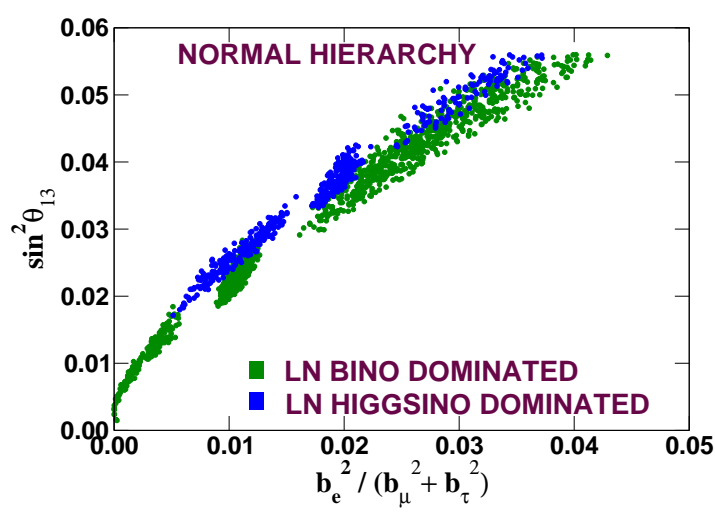

Figure 3: $\sin ^{2} \theta_{13}$ as a function of the ratio $\frac{b_{e}^{2}}{b_{\mu}^{2}+b_{\tau}^{2}}$. Values of other parameters are the same as in fig. 2 .

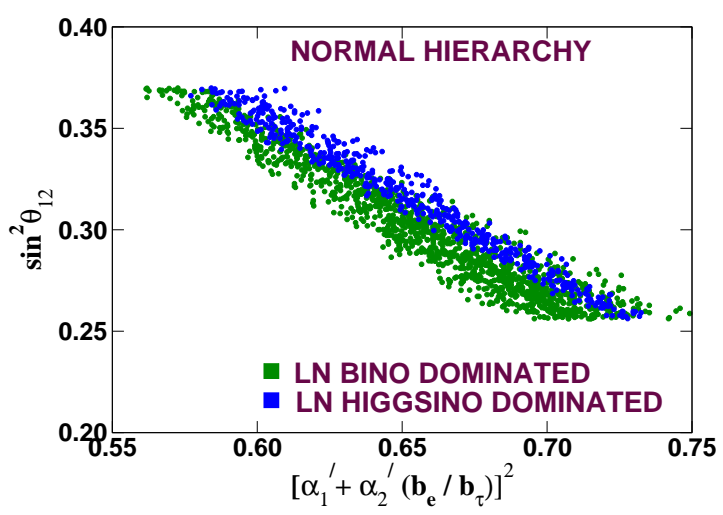

Figure 4: $\sin ^{2} \theta_{12}$ as a function of $\left(\alpha_{1}^{\prime}+\right.$ $\left.\alpha_{2}^{\prime} \frac{b_{e}}{b_{\tau}}\right)^{2}$. One can see that as $\left(\alpha_{1}^{\prime}+\alpha_{2}^{\prime} \frac{b_{e}}{b_{\tau}}\right)^{2} \rightarrow$ $0.50, \sin ^{2} \theta_{12}$ tends to 0.50 , as predicted by the analytical formula. Values of other parameters are the same as in fig. 2 .

Note that in some parts of these ranges we have considered a bino dominated lightest neutralino and in some parts we have taken a higgsino dominated lightest neutralino with 
some overlapping regions. The values of other parameters are chosen to be $m_{\tilde{L}}=400 \mathrm{GeV}$, $m_{\tilde{\nu}^{c}}=300 \mathrm{GeV}$ and $\tan \beta=10$. We have assumed that the phase $\delta$ appearing in the mixing matrix (5.14) is zero. One important thing to notice is that even with a flavour diagonal structure of the neutrino Yukawa couplings $Y_{\nu}$, one can obtain the required two large mixing angles for the neutrinos. The variations of other two mixing angles with the relevant parameters are shown in Figs. 3 and 4 .

In fig.5, we have shown the regions in the various $Y_{\nu}$ planes satisfying the three flavour global neutrino data. The values of other parameters are as in fig.2 for the case where the lightest neutralino is bino dominated. We can see from these figures that the allowed values of $Y_{\nu}$ s show a mild hierarchy such that $Y_{\nu}^{22}>Y_{\nu}^{11}>Y_{\nu}^{33}$.

Similar studies have been performed for the inverted hierarchical case and the allowed region shows that the magnitudes of the neutrino Yukawa couplings are larger compared to the case of normal hierarchical scheme of the neutrino masses with a different hierarchy among the $Y_{\nu}$ s themselves $\left(Y_{\nu}^{11}>Y_{\nu}^{22}>Y_{\nu}^{33}\right)$. In this case $\sin ^{2} \theta_{12}$ shows an increasing behaviour with the ratio $b_{e}^{2} / b_{\mu}^{2}$, similar to the one shown by $\sin ^{2} \theta_{23}$ with $b_{\mu}^{2} / b_{\tau}^{2}$ in the normal hierarchical scenario. On the other hand, $\sin ^{2} \theta_{23}$ shows a decreasing behaviour with $b_{\mu}^{2} / b_{\tau}^{2}$. In all these cases, the solar and atmospheric mass-squared differences are within the $3 \sigma$ limits.

The case of $\nu^{c}$ dominated lightest neutralino has also been studied and it shows a very interesting and different behaviour compared to the bino and higgsino dominated cases. In this case, the dominant contribution in the neutrino mass matrix (eq.(5.5)) comes from the term proportional to $a_{i} a_{j}$. The terms proportional to $b_{i} b_{j}$ should be considered as a perturbation. Hence, in the normal hierarchical scenario of neutrino masses, one would expect that $\sin ^{2} \theta_{23}$ is proportional to $a_{\mu}^{2} / a_{\tau}^{2}$. This is exactly what we see in fig.6.

Note that for $a_{\mu}^{2}=a_{\tau}^{2}$, the mixing becomes maximal. On the other hand, the solar mixing angle is controlled mostly by the quantity $b_{e}^{2} / b_{\mu}^{2}$ and shows an increasing behaviour with this ratio. In the case of inverted hierarchical scenario of neutrino masses, $\sin ^{2} \theta_{23}$ shows a decreasing behaviour with the ratio $b_{\mu}^{2} / b_{\tau}^{2}$ whereas $\sin ^{2} \theta_{12}$ shows an increasing pattern with $b_{e}^{2} / b_{\mu}^{2}$. However, we do not show these plots here.

\section{Decays of the lightest neutralino}

Let us now look at some decay processes which can be considered as the typical consequence of this model. It is obvious that because of the R-parity violation there will be no stable lightest supersymmetric particle (LSP) present in this model. Here we consider the case where the lightest neutralino ( $\tilde{\chi}_{7}^{0}$ in our notation to be described below) is the LSP (or NLSP in some cases) and study its decay pattern in the R-parity violating channels. In particular, we will consider the case where $m_{\tilde{\chi}_{7}^{0}}>m_{W^{ \pm}}$, so that the three-body decays are less important compared to the two-body decays $\tilde{\chi}_{7}^{0} \rightarrow Z+\nu_{e, \mu, \tau}, \tilde{\chi}_{7}^{0} \rightarrow W^{ \pm}+e^{\mp}$, $\tilde{\chi}_{7}^{0} \rightarrow W^{ \pm}+\mu^{\mp}$, and $\tilde{\chi}_{7}^{0} \rightarrow W^{ \pm}+\tau^{\mp}$. The required Feynman rules for the computation of the decay of the lightest neutralino are given in the Appendix B. Let us also remark that the lightest neutralino LSP can also decay to $h+\nu$, if it is kinematically allowed, 

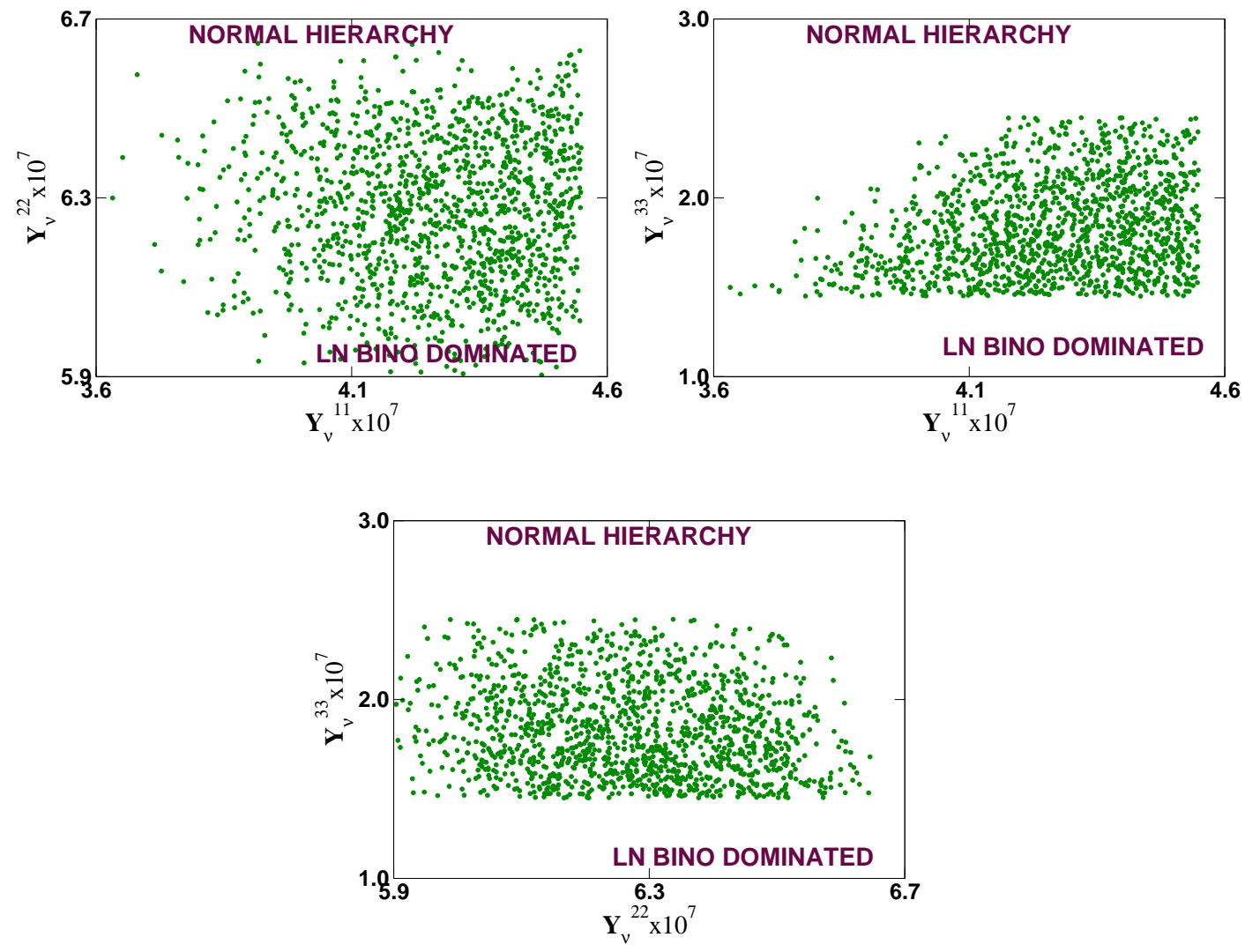

Figure 5: Plots for normal hierarchical scheme of neutrino mass in $Y_{\nu}^{11}-Y_{\nu}^{22}, Y_{\nu}^{11}-Y_{\nu}^{33}$ and $Y_{\nu}^{22}-Y_{\nu}^{33}$ plane when the lightest neutralino (LN) is bino dominated.

where $h$ is the MSSM-like lightest Higgs boson. However, for our illustration purposes we have considered the mass of the lightest neutralino in such a way that this decay is either kinematically forbidden or very much suppressed (assuming a lower bound on the mass of $h$ to be $114 \mathrm{GeV}$ ). Even if this decay branching ratio is slightly larger, it is usually smaller than the branching ratios in the $\left(\ell_{i}+W\right)$ channel. Hence, this will not affect our conclusions regarding the ratios of branching ratios in the charged lepton channel $\left(\ell_{i}+W\right)$, to be discussed later. The lightest neutralino decay $\tilde{\chi}_{7}^{0} \rightarrow \nu+\tilde{\nu}^{c}$, where $\tilde{\nu}^{c}$ is the scalar partner of the gauge singlet neutrino $\nu^{c}$, is always very suppressed. We will discuss more on this when we consider a $\nu^{c}$ dominated lightest neutralino.

Consider the following decay process

$$
\tilde{\chi}_{i} \longrightarrow \tilde{\chi}_{j}+V
$$

where $\tilde{\chi}_{i(j)}$ is either a neutralino or chargino, with mass $m_{i(j)}$ and $V$ is the gauge boson which is either $W^{ \pm}$or $Z$, with mass $m_{v}$. The masses $m_{i}$ and $m_{j}$ are positive.

The decay width for this process in eq.(6.1) is given by [23, 24]

$$
\Gamma\left(\tilde{\chi}_{i} \longrightarrow \tilde{\chi}_{j}+V\right)=\frac{g^{2} \mathcal{K}^{1 / 2}}{32 \pi m_{i}^{3} m_{W}^{2}} \times\left\{\left(G_{L}^{2}+G_{R}^{2}\right) \mathcal{F}-G_{L}^{*} G_{R} \mathcal{G}\right\}
$$


where $\mathcal{F}, \mathcal{G}$ are functions of $m_{i}, m_{j}, m_{v}$ and given by

$$
\begin{aligned}
& \mathcal{F}\left(m_{i}, m_{j}, m_{v}\right)=\mathcal{K}+3 m_{v}^{2}\left(m_{i}^{2}+m_{j}^{2}-m_{v}^{2}\right), \\
& \mathcal{G}\left(m_{i}, m_{j}, m_{v}\right)=12 \epsilon_{i} \epsilon_{j} m_{i} m_{j} m_{v}^{2},
\end{aligned}
$$

with $\epsilon_{i}(j)$ carrying the actual signs $( \pm 1)$ of the neutralino masses. The chargino masses must be positive. The kinematical factor $\mathcal{K}$ is given by

$$
\mathcal{K}\left(m_{i}^{2}, m_{j}^{2}, m_{v}^{2}\right)=\left(m_{i}^{2}+m_{j}^{2}-m_{v}^{2}\right)^{2}-4 m_{i}^{2} m_{j}^{2} .
$$

In order to derive eq.(6.2), we have used the relation $m_{W}^{2}=m_{Z}^{2} \cos ^{2} \theta_{W}$ and since $\left\langle\tilde{\nu}_{i}\right\rangle<<v_{1}, v_{2}$, some of the MSSM relations still hold good. The factors $G_{L}, G_{R}$ are given here for some possible decay modes

$$
\begin{aligned}
& \text { For } \quad \tilde{\chi}_{i}^{0} \longrightarrow \tilde{\chi}_{j}^{0} Z \\
& G_{L}=O_{j i}^{\prime \prime L}, \quad G_{R}=O_{j i}^{\prime \prime R}, \\
& \text { For } \quad \tilde{\chi}_{i}^{0} \longrightarrow \tilde{\chi}_{j}^{+} W^{-} \\
& G_{L}=O_{i j}^{L}, \quad G_{R}=O_{i j}^{R},
\end{aligned}
$$

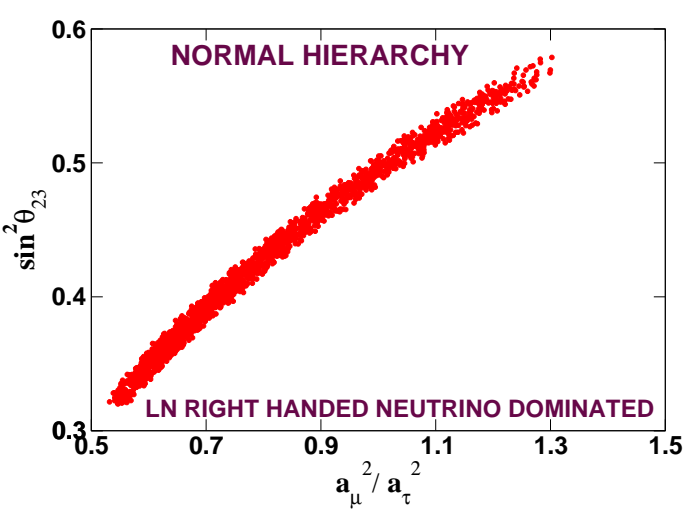

(6.4) Figure 6: Scatter plot of the neutrino mixing angle $\sin ^{2} \theta_{23}$ as a function of the ratio $\frac{a_{\mu}^{2}}{a_{\tau}^{2}}$. Values of other parameters are described in the text. The lightest neutralino (LN) is $\nu^{c}$ dominated.

Now consider the following decays

$$
\begin{aligned}
& \tilde{\chi}_{L N}^{0} \longrightarrow Z+\nu, \\
& \tilde{\chi}_{L N}^{0} \longrightarrow W^{ \pm}+\ell^{\mp},
\end{aligned}
$$

where $\tilde{\chi}_{L N}^{0}$ stands for lightest neutralino and $\ell=e, \mu, \tau$. At this stage let us discuss our notation and convention for these decays. The neutralino mass matrix is a $10 \times 10$ mass matrix which includes the left handed as well as the gauge-singlet neutrinos. If the mass eigenvalues of this matrix are arranged in the descending order then the three lightest eigenvalues of this $10 \times 10$ neutralino mass matrix would correspond to the three light neutrinos. Out of the remaining seven heavy eigenvalues, the lightest one is denoted as the lightest neutralino. Thus, in our notation $\tilde{\chi}_{7}^{0}$ is the lightest neutralino and $\tilde{\chi}_{j+7}^{0}$, where $j=1,2,3$ correspond to the three light neutrinos. Similarly, for the chargino masses, $\tilde{\chi}_{l+2}^{ \pm}$ $(l=1,2,3)$ corresponds to the charged leptons $e, \mu, \tau$. 
So for $\tilde{\chi}_{L N}^{0} \rightarrow Z+\nu$, which is also $\tilde{\chi}_{7}^{0} \rightarrow Z+\tilde{\chi}_{j+7}^{0}(j=1,2,3)$, one gets from eq.(6.4) and eq.(B.5)

$$
\begin{aligned}
G_{L} & =-\frac{1}{2} N_{j+7,3} N_{73}^{*}+\frac{1}{2} N_{j+7,4} N_{74}^{*}-\frac{1}{2} N_{j+7, k+7} N_{7, k+7}^{*}, \\
G_{R} & =-G_{L}^{*},
\end{aligned}
$$

where $j, k=1,2,3$ and this in turn modifies eq.(6.2) as

$$
\Gamma\left(\tilde{\chi}_{7}^{0} \rightarrow Z+\tilde{\chi}_{j+7}^{0}\right)=\frac{g^{2} \mathcal{K}^{1 / 2}}{32 \pi m_{\tilde{\chi}_{7}^{0}}^{3} m_{W}^{2}} \times\left\{2 G_{L}^{2} \mathcal{F}+G_{L}^{*^{2}} \mathcal{G}\right\}
$$

with $m_{i}=m_{\tilde{\chi}_{7}^{0}}, m_{j}=m_{\nu} \approx 0$ and $m_{v}=m_{Z}$.

Let us now consider the other decay which is $\tilde{\chi}_{L N}^{0} \rightarrow W^{ \pm}+\ell^{\mp}$ or equivalently $\tilde{\chi}_{7}^{0} \rightarrow$ $W^{ \pm}+\tilde{\chi}_{j}^{\mp}(j=3,4,5)$.

For the process $\tilde{\chi}_{7}^{0} \rightarrow W^{-}+\tilde{\chi}_{j}^{+}$

$$
\begin{aligned}
& \Gamma\left(\tilde{\chi}_{7}^{0} \rightarrow W^{-}+\tilde{\chi}_{j}^{+}\right)=\frac{g^{2} \mathcal{K}^{1 / 2}}{32 \pi m_{\tilde{\chi}_{7}^{0}}^{3} m_{W}^{2}} \times\left\{\left(G_{L}^{2}+G_{R}^{2}\right) \mathcal{F}-G_{L}^{*} G_{R} \mathcal{G}\right\} \\
& G_{L}=N_{72} V_{j 1}^{*}-\frac{1}{\sqrt{2}} N_{74} V_{j 2}^{*}, \\
& G_{R}=N_{72}^{*} U_{j 1}+\frac{1}{\sqrt{2}} N_{73}^{*} U_{j 2}+\frac{1}{\sqrt{2}} N_{7, k+7}^{*} U_{j, k+2}, \\
& (k=1,2,3),
\end{aligned}
$$

where eq.(6.4) and eq.(B.8) has been used. The process $\tilde{\chi}_{7}^{0} \longrightarrow W^{+}+\tilde{\chi}_{j}^{-}$is obtained by charge conjugation of the process in eq.(6.8).

\subsection{Correlation between the lightest neutralino decays and neutrino mixing angles}

Correlations between the lightest neutralino decays and neutrino mixing angles will depend on the nature of the lightest neutralino as well as on the mass hierarchies of the neutrinos, i.e. whether we have a normal hierarchical pattern of neutrino masses or an inverted one. In this section we look into these possibilities in details and consider three different cases for the dominant component of the lightest neutralino. We consider that the lightest neutralino is (1) bino dominated, (2) higgsino dominated, and (3) $\nu^{c}$ dominated. For each of these cases we consider both the normal and the inverted hierarchical pattern of neutrino masses. We show that in these different cases, the ratio of branching ratios of certain decays of the lightest neutralino correlates with the neutrino mixing angles. In some cases the correlation is with the atmospheric and the reactor angle and in other cases the ratio of the branching ratios correlates with the solar mixing angle and in some cases there is no correlations at all. Let us now study these possibilities case by case. The interesting difference between this study and similar studies with bilinear R-parity violating scenario [25] in the MSSM is the presence of a gauge singlet neutrino dominated lightest neutralino. 
We will see later that in this case the results can be very different from the bino or higgsino dominated lightest neutralino. The lightest neutralino decays in neutrino mass models with spontaneous R-parity violation have been studied in ref.[26].

\subsubsection{Bino dominated lightest neutralino}

We will assume that the gaugino masses are unified at the grand unified theory (GUT) scale. At the EW scale the ratio of the $U(1)$ and $S U(2)$ gaugino masses are $M_{1} / M_{2}=1: 2$. If in addition, $M_{1}<\mu$ and the value of $\kappa$ is large (so that the effective gauge singlet neutrino mass $2 \kappa \nu^{c}$ is large), the lightest neutralino is essentially bino dominated and it is the LSP. First we consider the case when the composition of the lightest neutralino is such that, the bino-component $\left|N_{71}\right|^{2}>0.92$ and neutrino masses follow the normal hierarchical pattern. We have observed that for the bino dominated case, the lightest neutralino $\left(\tilde{\chi}_{7}^{0}\right)$ couplings to $\ell^{ \pm}-W^{\mp}$ pair (where $\ell=e, \mu$ or $\tau$ ) depend on the quantities $b_{i}$ along with a factor which is independent of various lepton generations. Naturally, we would expect that the ratios of various decay branching ratios such as $\operatorname{BR}\left(\tilde{\chi}_{7}^{0} \rightarrow e+W\right), \operatorname{BR}\left(\tilde{\chi}_{7}^{0} \rightarrow \mu+W\right)$, and $\operatorname{BR}\left(\tilde{\chi}_{7}^{0} \rightarrow \tau+W\right)$ show nice correlations with the quantities $b_{i}^{2} / b_{j}^{2}$ with i,j being $e, \mu$ or $\tau$. This feature is evident from fig.7. Here we have scanned the parameter space of the three neutrino Yukawa couplings with random values for a particular choice of the couplings $\lambda, \kappa$ and the associated soft SUSY breaking trilinear parameters, as well as other MSSM parameters. The trilinear soft parameters $A_{\nu}$ corresponding to $Y_{\nu}$ s also vary randomly in a certain range. In addition we have imposed the condition that the lightest neutralino (which is the LSP) is bino dominated and neutrino mass pattern is normal hierarchical.

We have checked that the correlations between the ratios of the lightest neutralino decay branching ratios and $b_{i}^{2} / b_{j}^{2}$ is more prominent with increasing bino component of the lightest neutralino. Note that when $\left(b_{i} / b_{j}\right)^{2} \rightarrow 1$ the ratios of branching ratios shown in fig.7 also tend to 1 . We have seen earlier that the neutrino mixing angles $\theta_{23}$ and $\theta_{13}$ also show nice correlation with the ratios $b_{\mu}^{2} / b_{\tau}^{2}$ and $b_{e}^{2} / b_{\tau}^{2}$, respectively (see Figs.2 and 3). Hence we would expect that the ratios of the branching ratios $\frac{\operatorname{BR}\left(\tilde{\chi}_{7}^{0} \rightarrow \mu W\right)}{\operatorname{BR}\left(\tilde{\chi}_{7}^{0} \rightarrow \tau W\right)}$ and $\frac{B R\left(\tilde{\chi}_{7}^{0} \longrightarrow e W\right)}{\sqrt{B R\left(\tilde{\chi}_{7}^{0} \longrightarrow \mu W\right)^{2}+B R\left(\tilde{\chi}_{7}^{0} \longrightarrow \tau W\right)^{2}}}$ show correlations with $\tan ^{2} \theta_{23}$ and $\tan ^{2} \theta_{13}$. These correlations are shown in fig.8. We have seen earlier (see eq.(5.12)) that with the normal hierarchical pattern of the neutrino masses, the atmospheric mass scale is determined by the quantity $\Omega_{b}=\sqrt{b_{e}^{2}+b_{\mu}^{2}+b_{\tau}^{2}}$. Naturally one would expect that the atmospheric and the reactor angles are correlated with the $\ell+W$ final states of the lightest neutralino decays and no correlation is expected for the solar angle. This is what we have observed numerically. Here we have considered the regions of the parameter space where the neutrino mass-squared differences and mixing angles are within the $3 \sigma$ allowed range shown in eqs.1.1)-(1.3). fig.8 also shows the model prediction for the ratios of branching ratios where the neutrino experimental data are satisfied. For our sample choice of parameters in fig.8, one would expect that the ratio $\frac{B R\left(\tilde{\chi}_{7}^{0} \longrightarrow \mu W\right)}{B R\left(\tilde{\chi}_{7}^{0} \longrightarrow \tau W\right)}$ should be in the range 0.45 to 1.25 . Similarly, the other ratio $\frac{B R\left(\tilde{\chi}_{7}^{0} \longrightarrow e W\right)}{\sqrt{B R\left(\tilde{\chi}_{7}^{0} \longrightarrow \mu W\right)^{2}+B R\left(\tilde{\chi}_{7}^{0} \longrightarrow \tau W\right)^{2}}}$ is expected in this case to be less 

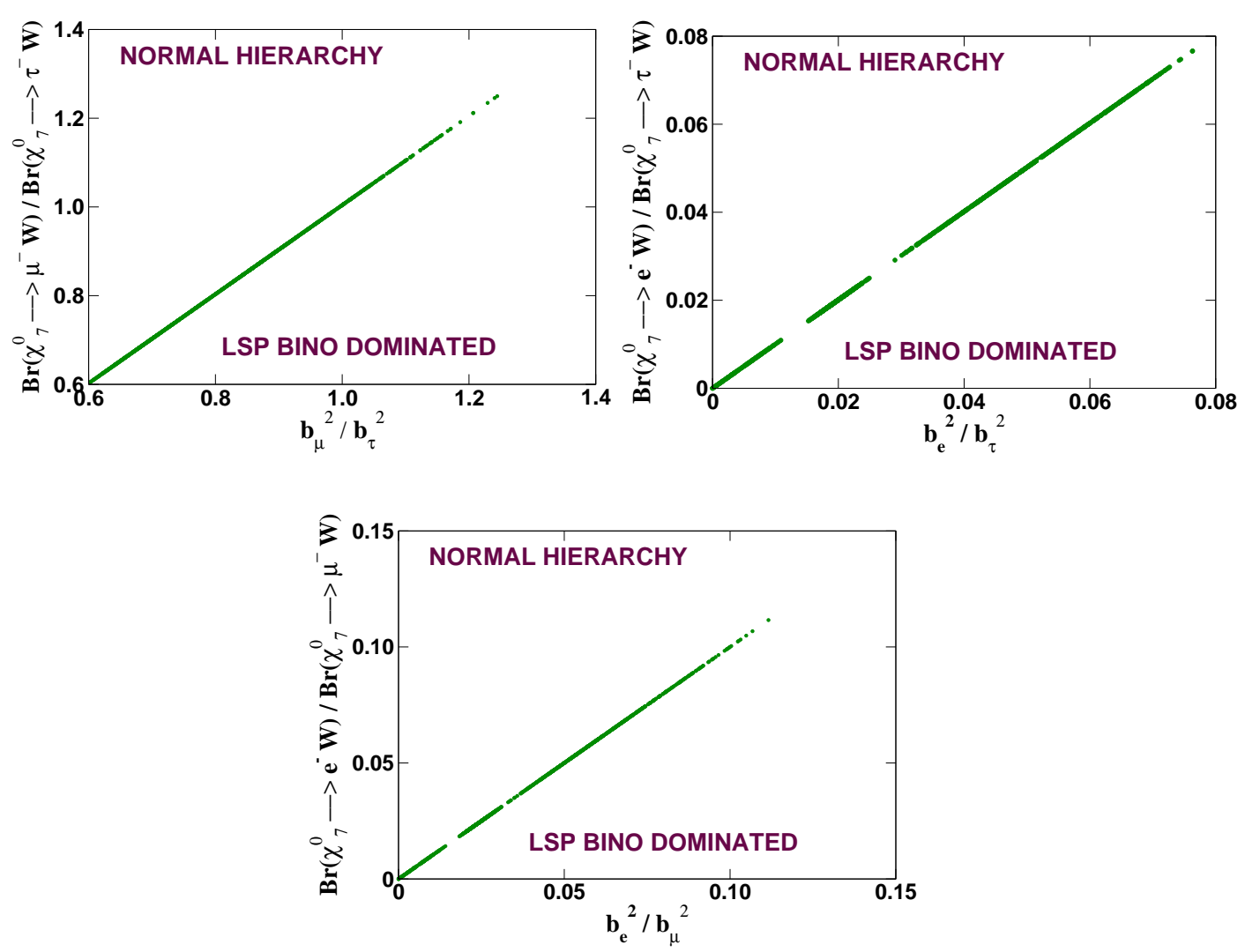

Figure 7: Ratio $\frac{B r\left(\chi_{7}^{0} \longrightarrow \ell_{i} W\right)}{\operatorname{Br}\left(\chi_{7}^{0} \longrightarrow \ell_{j} W\right)}$ versus $\frac{b_{i}^{2}}{b_{j}^{2}}$ plot for a bino like lightest neutralino (the LSP) with bino component, $\left|N_{71}\right|^{2}>0.92$, where $i, j, k=e, \mu, \tau$. Neutrino mass pattern is taken to be normal hierarchical. Choice of parameters are $M_{1}=110 \mathrm{GeV}, \lambda=0.13, \kappa=0.65, m_{\tilde{\nu}^{c}}=300 \mathrm{GeV}$ and $m_{\tilde{L}}=$ $400 \mathrm{GeV}$. Mass of the LSP is $106.9 \mathrm{GeV}$. The value of the $\mu$ parameter comes out to be -228.9 $\mathrm{GeV}$.

than 0.07 . We can also see from fig. 8 that the ratio of branching ratios in the $(\mu+W)$ and $(\tau+W)$ channels becomes almost equal for the maximal value of the atmospheric mixing angle $\left(\theta_{23}=45^{\circ}\right)$. On the other hand, we do not observe any correlation with the solar mixing angle $\theta_{12}$ since it is a complicated function of $a_{i}^{2}$ and $b_{i}^{2}$ (see eq. (5.31)).

In the case of inverted hierarchical mass pattern of the light neutrinos, the $\tilde{\chi}_{7}^{0}-\ell_{i}-W$ coupling is still controlled by the quantities $b_{i}^{2}$. Hence the ratios of the branching ratios discussed earlier, show nice correlations with $b_{i}^{2} / b_{j}^{2}$ (see fig.91). However, in this case the solar mixing angle shows some correlation with the ratio $\frac{B R\left(\tilde{\chi}_{7}^{0} \longrightarrow e W\right)}{\sqrt{B R\left(\tilde{\chi}_{7}^{0} \longrightarrow \mu W\right)^{2}+B R\left(\tilde{\chi}_{7}^{0} \longrightarrow \tau W\right)^{2}}}$. This is shown in fig.10. The correlation is not very sharp and some dispersion occurs due to the fact that the two heavier neutrino masses controlling the atmospheric mass scale and solar mass-squared difference are not completely determined by the quantities $b_{i}^{2}$ and there is some contribution of the quantities $a_{i}^{2}$, particularly for the second heavy neutrino mass eigenstate. The correlation of the ratio $\frac{B R\left(\tilde{\chi}_{7}^{0} \longrightarrow \mu W\right)}{B R\left(\tilde{\chi}_{7}^{0} \longrightarrow \tau W\right)}$ with $\tan ^{2} \theta_{23}$ shows a different behaviour compared to what we have seen in the case of normal hierarchical 

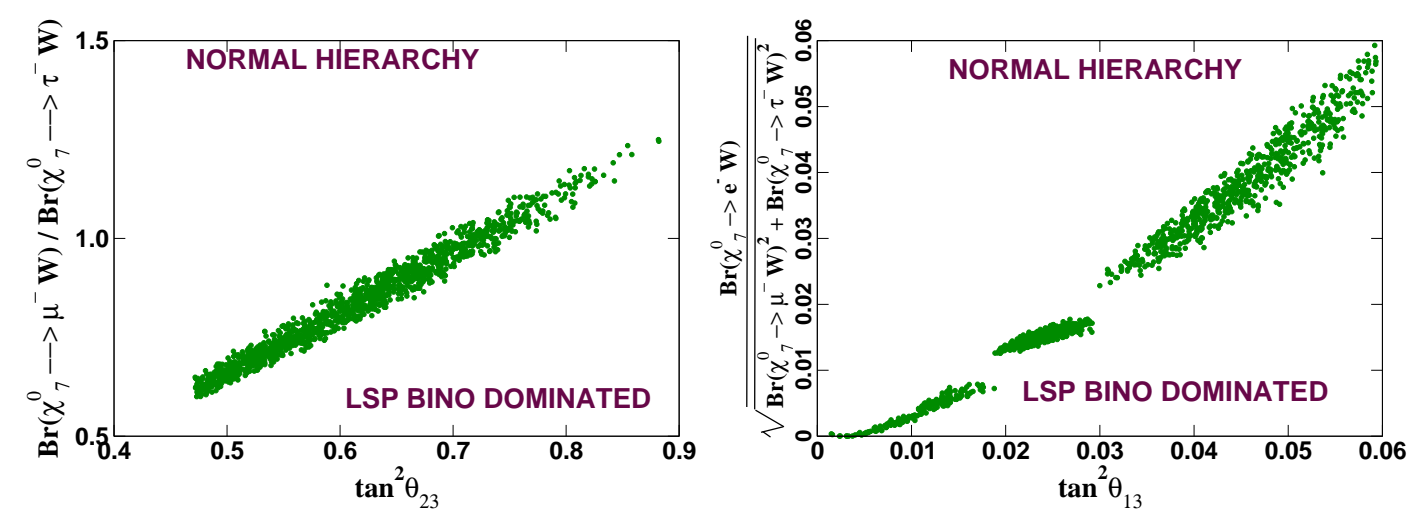

Figure 8: Ratio $\frac{B r\left(\chi_{7}^{0} \longrightarrow \mu W\right)}{B r\left(\chi_{7}^{0} \longrightarrow \tau W\right)}$ versus $\tan ^{2} \theta_{23} \quad$ (left), $\frac{B r\left(\chi_{7}^{0} \longrightarrow e W\right)}{\sqrt{B r\left(\chi_{7}^{0} \longrightarrow \mu W\right)^{2}+B r\left(\chi_{7}^{0} \longrightarrow \tau W\right)^{2}}}$ with $\tan ^{2} \theta_{13}$ (right) plot for a bino dominated lightest neutralino (the LSP) with bino component, $\left|N_{71}\right|^{2}>0.92$. Neutrino mass pattern is normal hierarchical. Choice of parameters are same as that of fig.7.

scenario. This is because in the case of inverted hierarchical mass pattern of the neutrinos, $\tan ^{2} \theta_{23}$ decreases with increasing $b_{\mu}^{2} / b_{\tau}^{2}$. One can observe from Figs. 8 and 10 that if the experimental value of the ratio $\frac{B R\left(\tilde{\chi}_{7}^{0} \longrightarrow e W\right)}{\sqrt{B R\left(\tilde{\chi}_{7}^{0} \longrightarrow \mu W\right)^{2}+B R\left(\tilde{\chi}_{7}^{0} \longrightarrow \tau W\right)^{2}}}$ is $\ll 1$ then that indicates a normal hierarchical neutrino mass pattern for a bino-dominated lightest neutralino LSP whereas a higher value $(\sim 1)$ of this ratio measured in experiments might indicate that the neutrino mass pattern is inverted hierarchical. Similarly a measurement of the ratio $\frac{B R\left(\tilde{\chi}_{7}^{0} \longrightarrow \mu W\right)}{B R\left(\tilde{\chi}_{7}^{0} \longrightarrow \tau W\right)}$ can also give an indication regarding the particular hierarchy of the neutrino mass pattern in the case of a bino dominated LSP.

\subsubsection{Higgsino dominated lightest neutralino}

When one considers higher values of the $U(1)$ gaugino mass $M_{1}$, i.e. $M_{1}>\mu$ and large value of $\kappa$ (so that the effective gauge singlet neutrino mass $2 \kappa \nu^{c}$ is large), the lightest neutralino is essentially higgsino dominated and it is the LSP. Naturally one needs to consider a small value of the coupling $\lambda$ so that the effective $\mu$ parameter $\left(\mu=3 \lambda \nu^{c}\right)$ is smaller. In order to look at the lightest neutralino decay branching ratios in this case, we consider a situation where the higgsino component in $\tilde{\chi}_{7}^{0}$ is $\left|N_{73}\right|^{2}+\left|N_{74}\right|^{2}>0.90$. As in the case of a bino dominated LSP, the generation dependence of the $\tilde{\chi}_{7}^{0}-\ell_{i}-W$ couplings comes through the quantities $b_{i}^{2}$. However, because of the large value of the $\tau$ Yukawa coupling, the higgsino $-\tau$ mixing is larger and as a result the partial decay width of $\tilde{\chi}_{7}^{0}$ into $(W+\tau)$ is larger than into $(W+\mu)$ and $(W+e)$. This feature is shown in fig.11, where the ratios of branching ratios are plotted against the quantities $b_{i}^{2} / b_{j}^{2}$. The domination of $\operatorname{BR}\left(\tilde{\chi}_{7}^{0} \rightarrow \tau+W\right)$ over the other two is clearly evident. Nevertheless, all the three ratios of branching ratios show sharp correlations with the corresponding $b_{i}^{2} / b_{j}^{2}$. In this figure the normal hierarchical pattern of the neutrino masses has been considered. As in the case of a bino LSP, here also the ratios $\frac{B R\left(\tilde{\chi}_{7}^{0} \longrightarrow \mu W\right)}{B R\left(\tilde{\chi}_{7}^{0} \longrightarrow \tau W\right)}$ and $\frac{B R\left(\tilde{\chi}_{7}^{0} \longrightarrow e W\right)}{\sqrt{B R\left(\tilde{\chi}_{7}^{0} \longrightarrow \mu W\right)^{2}+B R\left(\tilde{\chi}_{7}^{0} \longrightarrow \tau W\right)^{2}}}$ show nice correlations with neutrino mixing angles $\theta_{23}$ and $\theta_{13}$, respectively. This is shown in fig.12. 

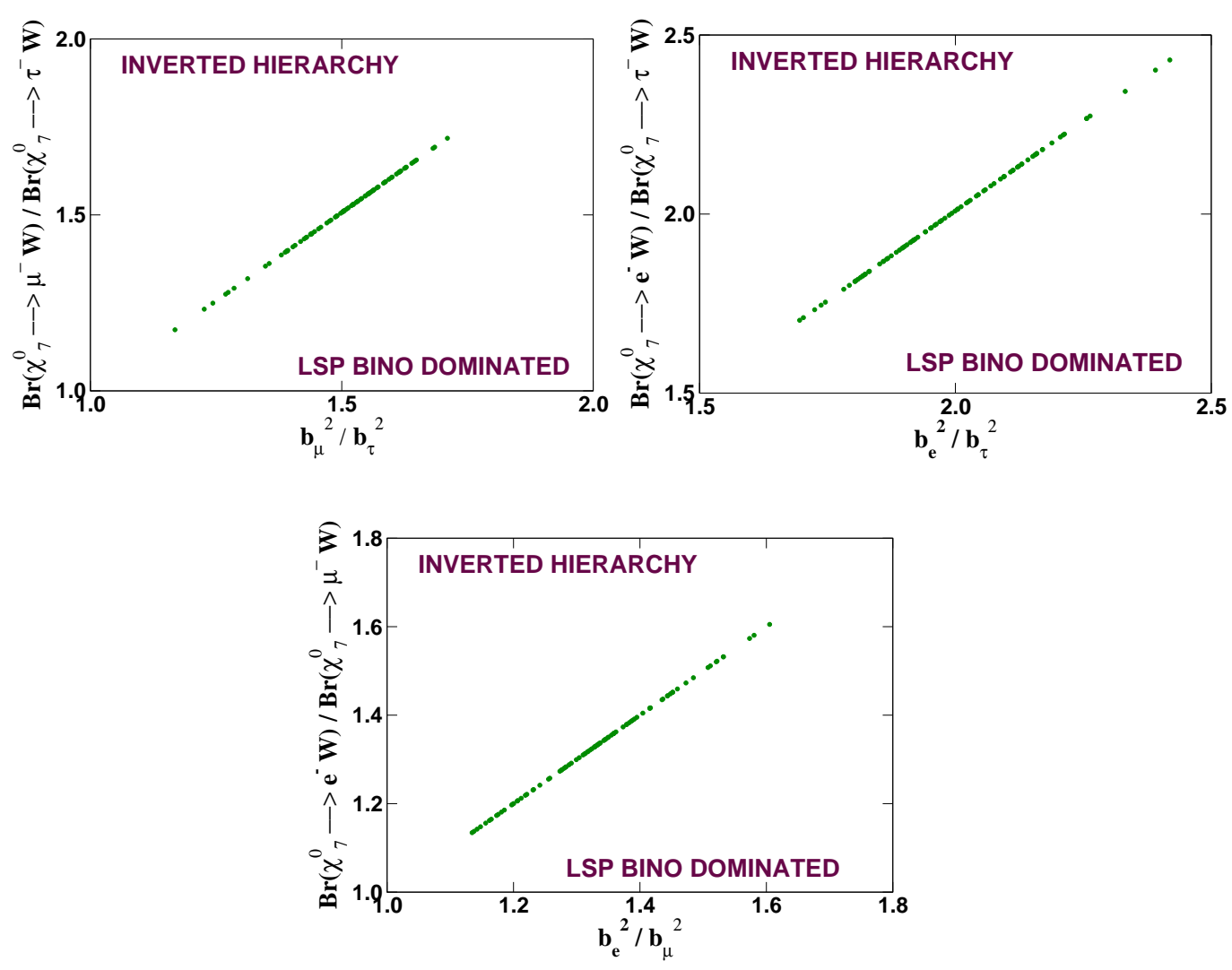

Figure 9: Ratio $\frac{B R\left(\tilde{\chi}_{7}^{0} \rightarrow \ell_{i}^{-} W\right)}{B R\left(\tilde{\chi}_{7}^{0} \longrightarrow \ell_{j}^{-} W\right)}$ versus $\frac{b_{i}^{2}}{b_{j}^{2}}$ plot for a bino like lightest neutralino (the LSP) with bino component $\left|N_{71}\right|^{2}>0.95$, where $i, j, k=e, \mu, \tau$. Neutrino mass pattern is inverted hierarchical. Choice of parameters are $M_{1}=105 \mathrm{GeV}, \lambda=0.15, \kappa=0.65, m_{\tilde{\nu}^{c}}=300 \mathrm{GeV}$ and $m_{\tilde{L}}=445 \mathrm{GeV}$. Mass of the LSP is $103.3 \mathrm{GeV}$. The value of the $\mu$ parameter comes out to be $-263.7 \mathrm{GeV}$.

However, in this case the predictions for these two ratios are very different from the bino LSP case. The expected value of the ratio $\frac{B R\left(\tilde{\chi}_{7}^{0} \longrightarrow \mu W\right)}{B R\left(\tilde{\chi}_{7}^{0} \longrightarrow \tau W\right)}$ is approximately between 0.05 and 0.10 in a region where one can accommodate the experimental neutrino data. Similarly, the predicted value of the ratio $\frac{B R\left(\tilde{\chi}_{7}^{0} \longrightarrow e W\right)}{\sqrt{B R\left(\tilde{\chi}_{7}^{0} \longrightarrow \mu W\right)^{2}+B R\left(\tilde{\chi}_{7}^{0} \longrightarrow \tau W\right)^{2}}}$ is $\leq 0.006$. On the other hand, there is no such correlations with the solar mixing angle $\theta_{12}$.

Similar correlations of the ratios of branching ratios with $b_{i}^{2} / b_{j}^{2}$ are also obtained for a higgsino dominated LSP in the case where the neutrino mass pattern is inverted hierarchical. Once again it shows that the $\tilde{\chi}_{7}^{0}$ decays to $(\tau+W)$ channel is dominant over the channels $(e+W)$ and $(\mu+W)$ for any values of $b_{i}^{2} / b_{j}^{2}$ because of the larger $\tau$ Yukawa 

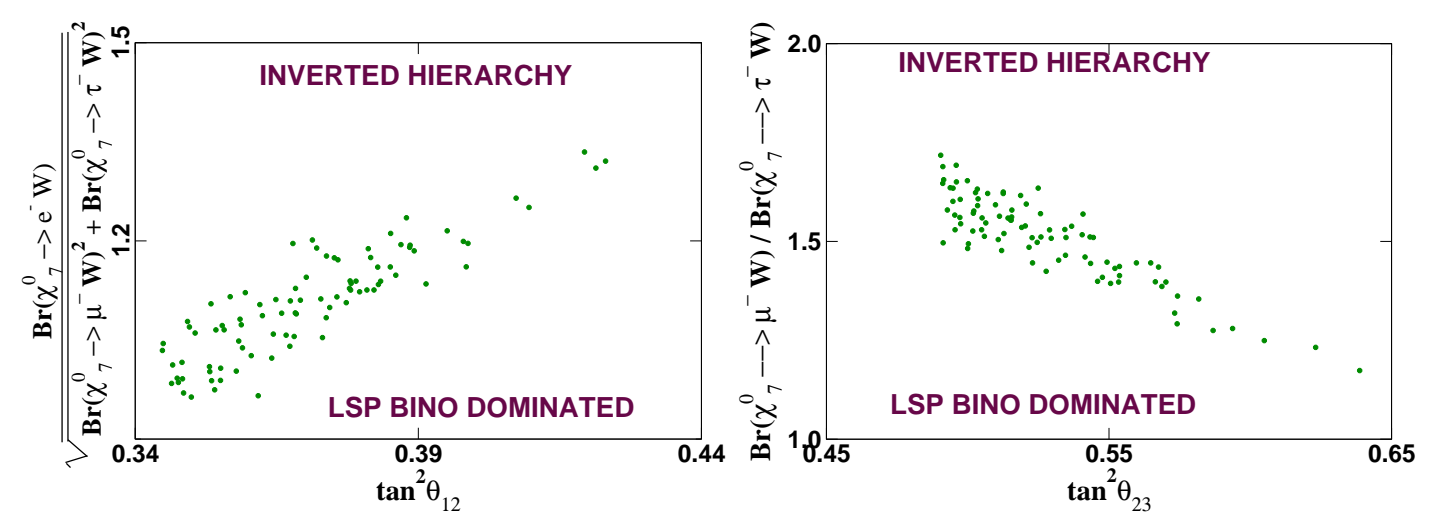

Figure 10: Ratio $\frac{B R\left(\tilde{\chi}_{7}^{0} \longrightarrow e W\right)}{\sqrt{B R\left(\tilde{\chi}_{7}^{0} \longrightarrow \mu W\right)^{2}+B R\left(\tilde{\chi}_{7}^{0} \longrightarrow \tau W\right)^{2}}}$ with $\tan ^{2} \theta_{12}$ (left) plot for a bino dominated lightest neutralino (LSP) with bino component $\left|N_{71}\right|^{2}>0.95$. In the right figure the ratio $\frac{B R\left(\tilde{\chi}_{7}^{0} \longrightarrow \mu W\right)}{B R\left(\tilde{\chi}_{7}^{0} \longrightarrow \tau W\right)}$ versus $\tan ^{2} \theta_{23}$ is plotted. Neutrino mass pattern is assumed to be inverted hierarchical. Choice of parameters are same as that of fig.9.

coupling. On the other hand, the correlations with the neutrino mixing angles show a behaviour similar to that of a bino LSP with inverted neutrino mass hierarchy though with much smaller values for the ratios $\frac{B R\left(\tilde{\chi}_{7}^{0} \rightarrow \mu W\right)}{B R\left(\tilde{\chi}_{7}^{0} \rightarrow \tau W\right)}$ and $\frac{B R\left(\tilde{\chi}_{7}^{0} \rightarrow e W\right)}{\sqrt{B R\left(\tilde{\chi}_{7}^{0} \rightarrow \mu W\right)^{2}+B r\left(\tilde{\chi}_{7}^{0} \rightarrow \tau W\right)^{2}}}$. These are shown in fig.13. Note that the correlations in this case are not very sharp, especially with $\tan ^{2} \theta_{12}$. Thus we see that small values of these ratios (for both normal and inverted hierarchy) are characteristic features of a higgsino dominated LSP in this model.

\subsection{3 $\nu^{c}$ dominated lightest neutralino}

Because of our choice of parameters i.e., a generation independent coupling $\kappa$ of the gauge singlet neutrinos and a common $\mathrm{VEV} \nu^{c}$, the three neutralino mass eigenstates which are predominantly gauge singlet neutrinos are essentially mass degenerate. There is a very small mass splitting due to mixing. However, unlike the case of a bino or higgsino dominated lightest neutralino, these $\nu^{c}$ dominated lightest neutralino states cannot be considered as the LSP. This is because in this case the lightest scalar (which is predominantly a gauge singlet sneutrino $\widetilde{\nu}^{c}$ ) is the lightest supersymmetric particle. This is very interesting since usually one does not get a $\tilde{\nu}^{c}$ as an LSP in a model where the gauge singlet neutrino superfield has a large Majorana mass term in the superpotential. However, in this case the effective Majorana mass term is at the EW scale and there is also a contribution from the trilinear scalar coupling $A_{\kappa} \kappa$ which keeps the mass of the singlet scalar sneutrino smaller. It is also very interesting to study the decay patterns of the lightest neutralino in this case since here one can probe the gauge singlet neutrino mass scales at the colliders.

Before discussing the decay patterns of the lightest neutralino which is $\nu^{c}$ dominated, let us say a few words regarding their production at the LHC. The direct production of $\nu^{c}$ 

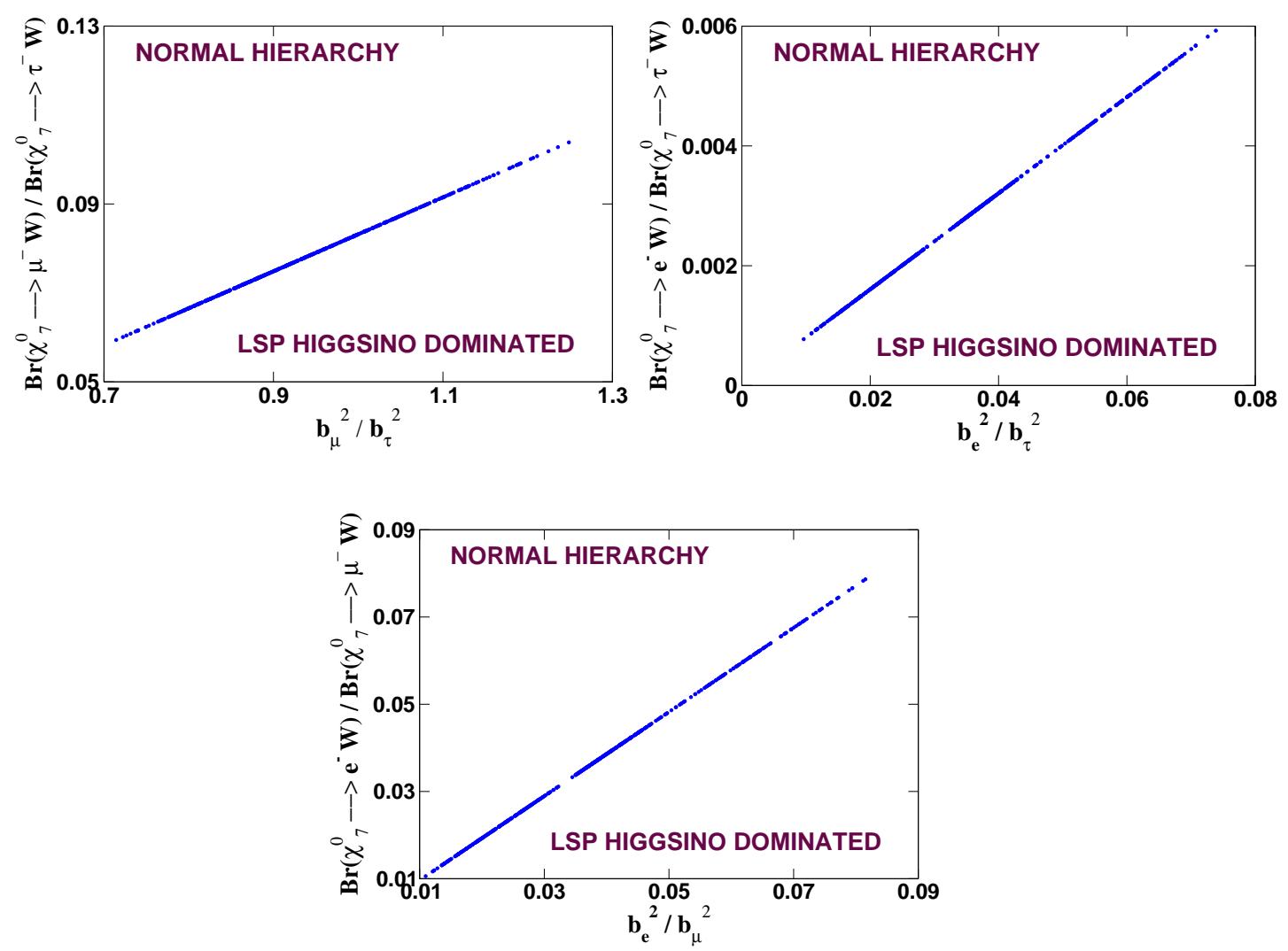

Figure 11: Ratio $\frac{B R\left(\tilde{\chi}_{7}^{0} \longrightarrow l_{i} W\right)}{B R\left(\tilde{\chi}_{7}^{0} \longrightarrow l_{j} W\right)}$ versus $\frac{b_{i}^{2}}{b_{j}^{2}}$ plot for a higgsino like LSP with higgsino component $\left(\left|N_{73}\right|^{2}+\left|N_{74}\right|^{2}\right)>0.95$, where $i, j, k=e, \mu, \tau$. Neutrino mass pattern is normal hierarchical. Choice of parameters are $M_{1}=325 \mathrm{GeV}, \lambda=0.06, \kappa=0.65, m_{\tilde{\nu}^{c}}=300 \mathrm{GeV}$ and $\mathrm{m}_{\tilde{\mathrm{L}}}=400 \mathrm{GeV}$. Mass of the LSP is $98.6 \mathrm{GeV}$. The value of the $\mu$ parameter comes out to be $-105.9 \mathrm{GeV}$.

(by $\nu^{c}$ we mean the $\nu^{c}$ dominated lightest neutralino in this section) is negligible because of the very small mixing with the MSSM neutralinos. Nevertheless, they can be produced at the end of the cascade decay chains of the squarks and gluinos at the LHC. For example, if the next-to-next-to-lightest SUSY particle (NNLSP) is higgsino dominated (this is the state above the three almost degenerate lightest neutralinos) and it has a non-negligible mixing with $\nu^{c}$ (remember that the higgsino- $\nu^{c}$ mixing occurs mainly because of the term $\lambda \hat{\nu}^{c} \hat{H}_{1} \hat{H}_{2}$ in the superpotential), then the branching ratio of the decay $\tilde{H} \rightarrow Z+\nu^{c}$ can be larger than the branching ratios in the $\ell W$ and $\nu Z$ channels. This way one can produce $\nu^{c}$ dominated lightest neutralino. Similarly, a higgsino dominated lighter chargino can also produce gauge singlet neutrinos. Another way of producing $\nu^{c}$ is through the decay of an NNLSP $\tilde{\tau}_{1}$, such as $\tilde{\tau}_{1} \rightarrow \tau+\nu^{c}$. A detailed discussion of these issues is beyond the scope of the present paper and we hope to come back to this in a future publication 27.

When one considers higher value of the gaugino mass, i.e. $M_{1}>\mu$ and a small value of the coupling $\kappa$ (so that the effective Majorana mass of $\nu^{c}$ is small, i.e. $2 \kappa \nu^{c}<\mu$ ), 

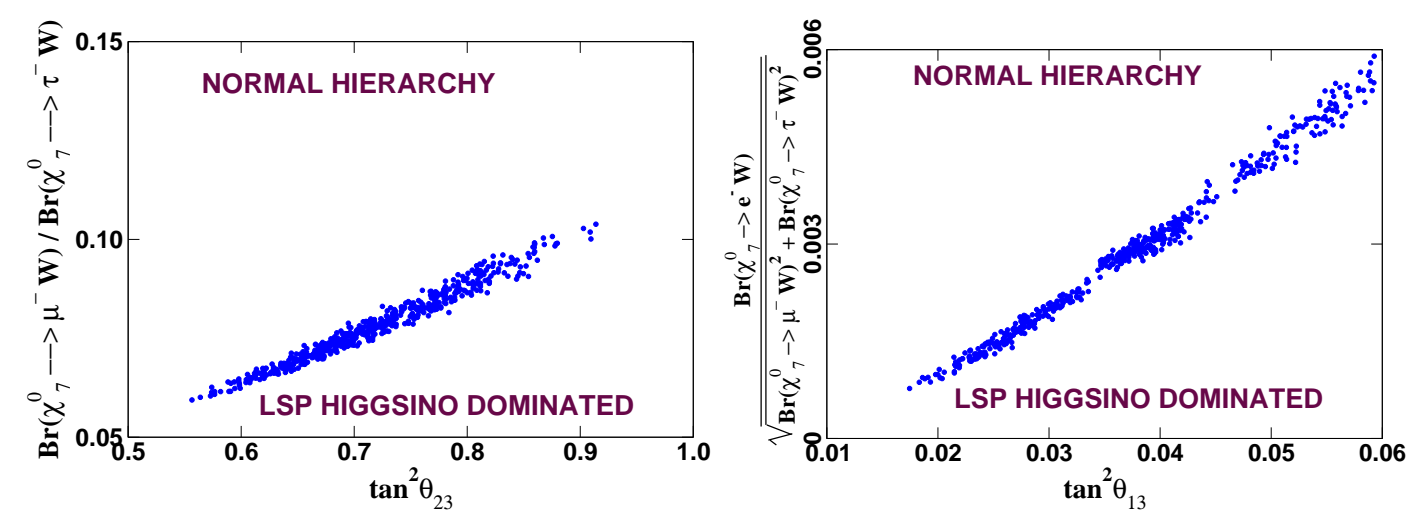

Figure 12: Ratio $\frac{B R\left(\tilde{\chi}_{7}^{0} \longrightarrow \mu W\right)}{B R\left(\tilde{\chi}_{7}^{0} \longrightarrow \tau W\right)}$ versus $\tan ^{2} \theta_{23} \quad($ left $), \frac{B R\left(\tilde{\chi}_{7}^{0} \longrightarrow e W\right)}{\sqrt{B R\left(\tilde{\chi}_{7}^{0} \longrightarrow \mu W\right)^{2}+B R\left(\tilde{\chi}_{7}^{0} \longrightarrow \tau W\right)^{2}}}$ with $\tan ^{2} \theta_{13}$ (right) plot for a higgsino LSP with higgsino component $\left(\left|N_{73}\right|^{2}+\left|N_{74}\right|^{2}\right)>0.95$. Neutrino mass pattern is normal hierarchical. Choice of parameters are same as that of fig.11.
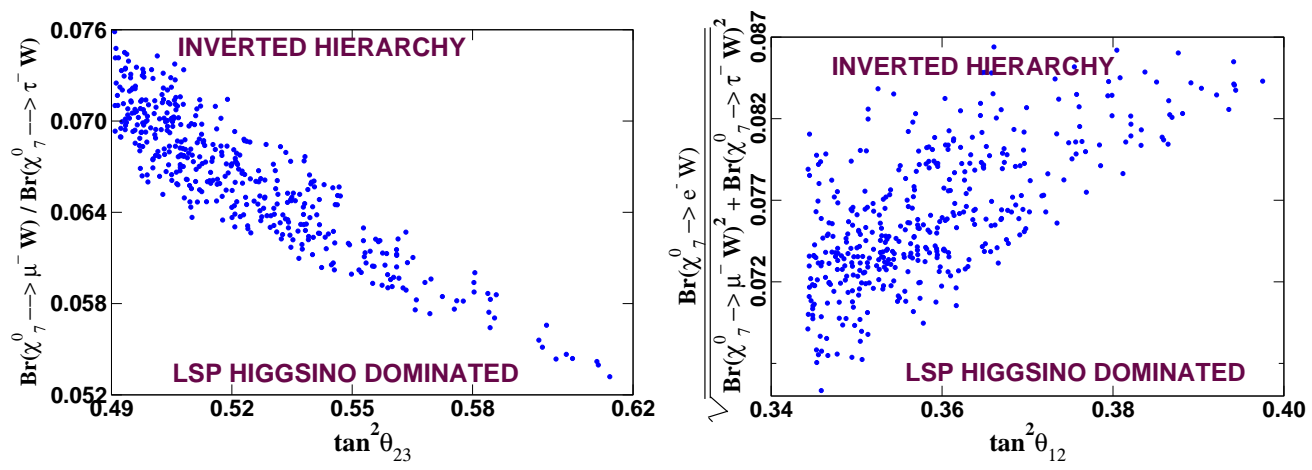

Figure 13: Ratio $\frac{B R\left(\tilde{\chi}_{7}^{0} \longrightarrow \mu W\right)}{B R\left(\tilde{\chi}_{7}^{0} \longrightarrow \tau W\right)}$ versus $\tan ^{2} \theta_{23} \quad$ (left), $\frac{B R\left(\tilde{\chi}_{7}^{0} \longrightarrow e W\right)}{\sqrt{B R\left(\tilde{\chi}_{7}^{0} \longrightarrow \mu W\right)^{2}+B r\left(\tilde{\chi}_{7}^{0} \longrightarrow \tau W\right)^{2}}}$ with $\tan ^{2} \theta_{12}$ (right) plot for a higgsino LSP with higgsino component $\left(\left|N_{73}\right|^{2}+\left|N_{74}\right|^{2}\right)>0.95$. Neutrino mass pattern is inverted hierarchical. Choice of parameters are $M_{1}=490 \mathrm{GeV}$, $\lambda=0.07, \kappa=0.65, m_{\tilde{\nu}^{c}}=320 \mathrm{GeV}$ and $\mathrm{m}_{\tilde{\mathrm{L}}}=430 \mathrm{GeV}$. Mass of the LSP is $110.8 \mathrm{GeV}$. The value of the $\mu$ parameter comes out to be $-115.3 \mathrm{GeV}$.

the lightest neutralino is essentially $\nu^{c}$ dominated. As we have mentioned earlier, in this case the LSP is the scalar partner of $\nu^{c}$, i.e. $\tilde{\nu}^{c}$. However, the decay of $\nu^{c}$ into $\nu+\tilde{\nu}^{c}$ is suppressed compared to the decays $\nu^{c} \rightarrow \ell_{i}+W$ and $\nu^{c} \rightarrow \nu_{i}+Z$ that we have considered so far. Because of this in this section we will neglect the decay $\nu^{c} \rightarrow \nu+\tilde{\nu}^{c}$ while discussing the correlation of the lightest neutralino $\left(\tilde{\chi}_{7}^{0}\right)$ decays with the neutrino mixing angles.

In this case the coupling of the lightest neutralino $\left(\tilde{\chi}_{7}^{0}\right)$ with $\ell_{i}-W$ pair depends on the $\nu^{c}$ content of $\tilde{\chi}_{7}^{0}$. Note that the $\nu^{c}$ has a very small mixing with the MSSM neutralino states. However, in some cases the $\nu^{c}$ dominated lightest neutralino can have a nonnegligible higgsino component. In such cases the coupling $\tilde{\chi}_{7}^{0}-\ell_{i}-W$ depends mainly on the quantities $b_{i}$. On the other hand, if $\tilde{\chi}_{7}^{0}$ is very highly dominated by $\nu^{c}$, then the coupling $\tilde{\chi}_{7}^{0}-\ell_{i}-W$ has a nice correlation with the quantities $a_{i}$. So in order to study the 

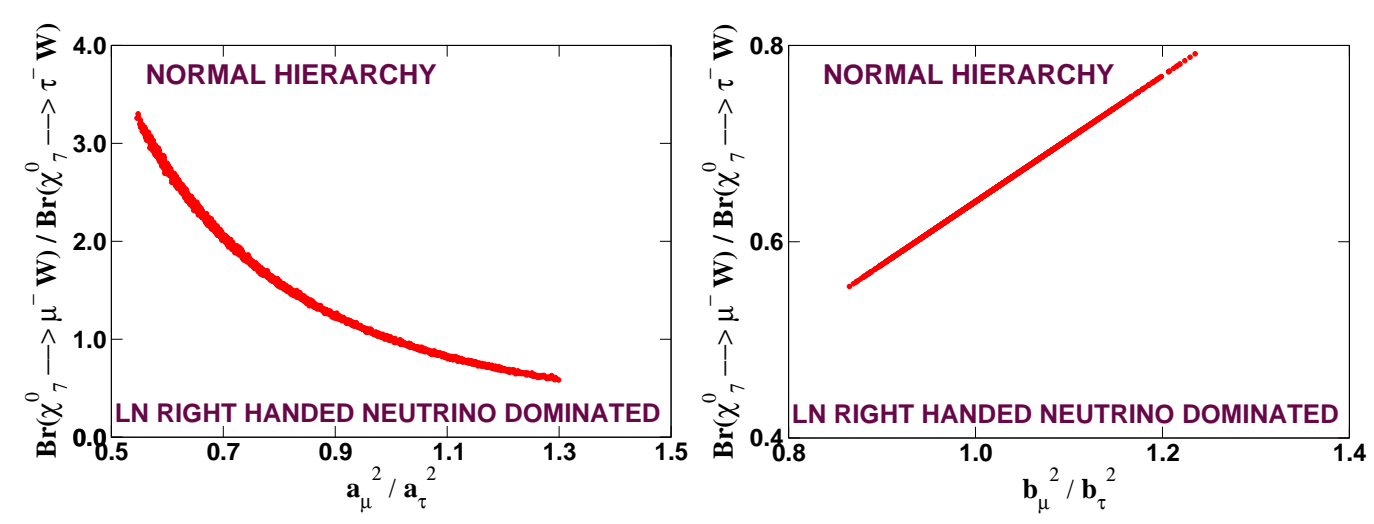

Figure 14: Ratio $\frac{B R\left(\tilde{\chi}_{-}^{0} \longrightarrow \mu W\right)}{B R\left(\tilde{\chi}_{7}^{0} \longrightarrow \tau W\right)}$ versus $\frac{a_{\mu}^{2}}{a_{\tau}^{2}}$ (left) and versus $\frac{b_{\mu}^{2}}{b_{\tau}^{2}}$ (right) plot for a $\nu^{c}$ like lightest neutralino $\left(\tilde{\chi}_{7}^{0}\right)$ with $\nu^{c}$ component $\left(\left|N_{75}\right|^{2}+\left|N_{76}\right|^{2}+\left|N_{77}\right|^{2}\right)>0.99$, (left) and $>0.97$ (right). Neutrino mass pattern is normal hierarchical. Choice of parameters are for (left) $M_{1}=405 \mathrm{GeV}$, $\lambda=0.29, \kappa=0.07,\left(A_{\lambda} \lambda\right)=-8.2 \mathrm{TeV} \times \lambda,\left(A_{\kappa} \kappa\right)=165 \mathrm{GeV} \times \kappa, m_{\tilde{\nu}^{c}}=50 \mathrm{GeV}$ and $\mathrm{m}_{\tilde{\mathrm{L}}}=825 \mathrm{GeV}$ and for (right) $M_{1}=405 \mathrm{GeV}, \lambda=0.10, \kappa=0.07,\left(A_{\lambda} \lambda\right)=-2 \mathrm{TeV} \times \lambda,\left(A_{\kappa} \kappa\right)=165 \mathrm{GeV} \times$ $\kappa, m_{\tilde{\nu}^{c}}=50 \mathrm{GeV}$ and $m_{\tilde{L}}=825 \mathrm{GeV}$. Mass of the lightest neutralino is $129.4 \mathrm{GeV}$ (left) and $119.8 \mathrm{GeV}$ (right) respectively. The values of the $\mu$ parameter are $-803.9 \mathrm{GeV}$ and $-258.8 \mathrm{GeV}$, respectively.

decay correlations of the $\nu^{c}$ dominated lightest neutralino, we consider two cases (i) $\nu^{c}$ component is $>0.99$, and (ii) $\nu^{c}$ component is $>0.97$ with some non-negligible higgsino admixture.

The correlations of the decay branching ratio $\frac{B R\left(\tilde{\chi}_{7}^{0} \longrightarrow \mu W\right)}{B R\left(\tilde{\chi}_{7}^{0} \longrightarrow \tau W\right)}$ are shown in fig.14 for the cases (i) and (ii) mentioned above. As we have explained already, this figure demonstrates that in case (i) the ratio of the branching ratio is dependent on the quantity $a_{\mu}^{2} / a_{\tau}^{2}$ whereas in case (ii) this ratio is correlated with $b_{\mu}^{2} / b_{\tau}^{2}$ though there is some suppression due to large $\tau$ Yukawa coupling.

Similar calculations were performed also for the other ratios discussed earlier. For example, in fig.15 we have shown the variations of the ratio $\frac{B R\left(\tilde{\chi}_{7}^{0} \longrightarrow e W\right)}{B R\left(\tilde{\chi}_{7}^{0} \longrightarrow \mu W\right)}$ as functions of $\frac{a_{e}^{2}}{a_{\mu}^{2}}$ and $\frac{b_{e}^{2}}{b_{\mu}^{2}}$ for the cases (i) and (ii), respectively. The variation with $\frac{a_{e}^{2}}{a_{\mu}^{2}}$ is not sharp and dispersive in nature whereas the variation with $\frac{b_{e}^{2}}{b_{\mu}^{2}}$ is very sharp and shows that in this case the relevant couplings are proportional to $b_{e}$ and $b_{\mu}$, respectively.

On the other hand, in case (i) only $\tan ^{2} \theta_{23}$ shows a nice correlation with the ratio $\frac{B R\left(\tilde{\chi}_{7}^{0} \longrightarrow \mu W\right)}{B R\left(\tilde{\chi}_{7}^{0} \longrightarrow \tau W\right)}$ (see fig.16) and $\tan ^{2} \theta_{12}$ or $\tan ^{2} \theta_{13}$ does not show any correlation with the other ratio. The non-linear behaviour of the ratios of branching ratios in case(i) is due to the fact that the parameters $Y_{\nu} \mathrm{s}$ (which control the $a_{i}$ ) appear both in the neutralino and chargino mass matrices. The charged lepton Yukawa couplings also play a role in determining the ratios. One can also see that the prediction for this ratio of branching ratio for case (i), as shown in fig.16, is in the range 0.5-3.5, which is larger compared to 

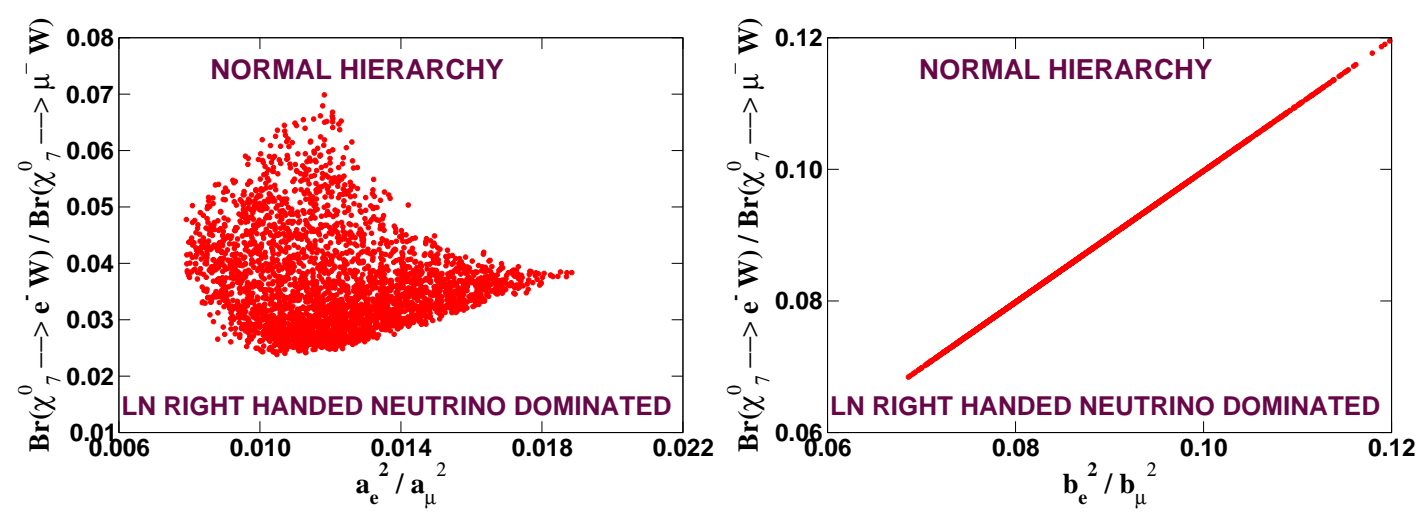

Figure 15: Ratio $\frac{B R\left(\tilde{\chi}_{7}^{0} \longrightarrow e W\right)}{B R\left(\tilde{\chi}_{7}^{0} \longrightarrow \mu W\right)}$ versus $\frac{a_{e}^{2}}{a_{\mu}^{2}}$ (left) and versus $\frac{b_{e}^{2}}{b_{\mu}^{2}}$ (right) plot for a $\nu^{c}$ like lightest neutralino $\left(\tilde{\chi}_{7}^{0}\right)$ with $\nu^{c}$ component $\left(\left|N_{75}\right|^{2}+\left|N_{76}\right|^{2}+\left|N_{77}\right|^{2}\right)>0.99$ (left), and $>0.97$ (right). Neutrino mass pattern is normal hierarchical. Choice of parameters are same as that of fig.14.
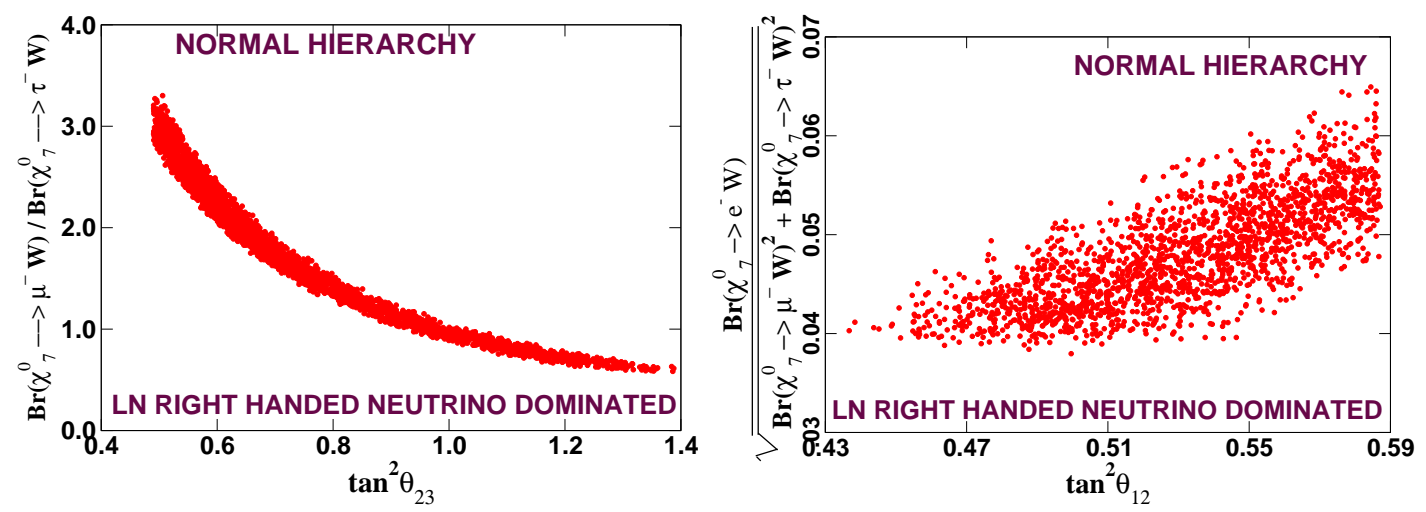

Figure 16: Ratio $\frac{B R\left(\tilde{\chi}_{7}^{0} \longrightarrow \mu W\right)}{B R\left(\tilde{\chi}_{7}^{0} \longrightarrow \tau W\right)}$ versus $\tan ^{2} \theta_{23} \quad$ (left), $\frac{B R\left(\tilde{\chi}_{7}^{0} \longrightarrow e W\right)}{\sqrt{B R\left(\tilde{\chi}_{7}^{0} \longrightarrow \mu W\right)^{2}+B R\left(\tilde{\chi}_{7}^{0} \longrightarrow \tau W\right)^{2}}}$ with $\tan ^{2} \theta_{12}$ (right) plot for a $\nu^{c}$ dominated lightest neutralino with $\nu^{c}$ component $\left(\left|N_{75}\right|^{2}+\left|N_{76}\right|^{2}+\right.$ $\left.\left|N_{77}\right|^{2}\right)>0.99$ (left) and $>0.97$ (right). Neutrino mass pattern is normal hierarchical. Choice of parameters are same as that of fig.14.

the bino dominated or higgsino dominated cases for both normal and inverted hierarchical pattern of neutrino masses. Also, the nature of this variation is similar to what we see with the inverted hierarchical pattern of neutrino masses in the bino or higgsino dominated cases.

In case (ii) none of the neutrino mixing angles show very good correlations with the ratios of branching ratios that we have been discussing. However, one can still observe some kind of a correlation of the ratio $\frac{B R\left(\tilde{\chi}_{7}^{0} \longrightarrow e W\right)}{\sqrt{B R\left(\tilde{\chi}_{7}^{0} \longrightarrow \mu W\right)^{2}+B R\left(\tilde{\chi}_{7}^{0} \longrightarrow \tau W\right)^{2}}}$ with $\tan ^{2} \theta_{12}$. The prediction for this ratio from the neutrino data is on the smaller side $(\sim 0.07)$. 

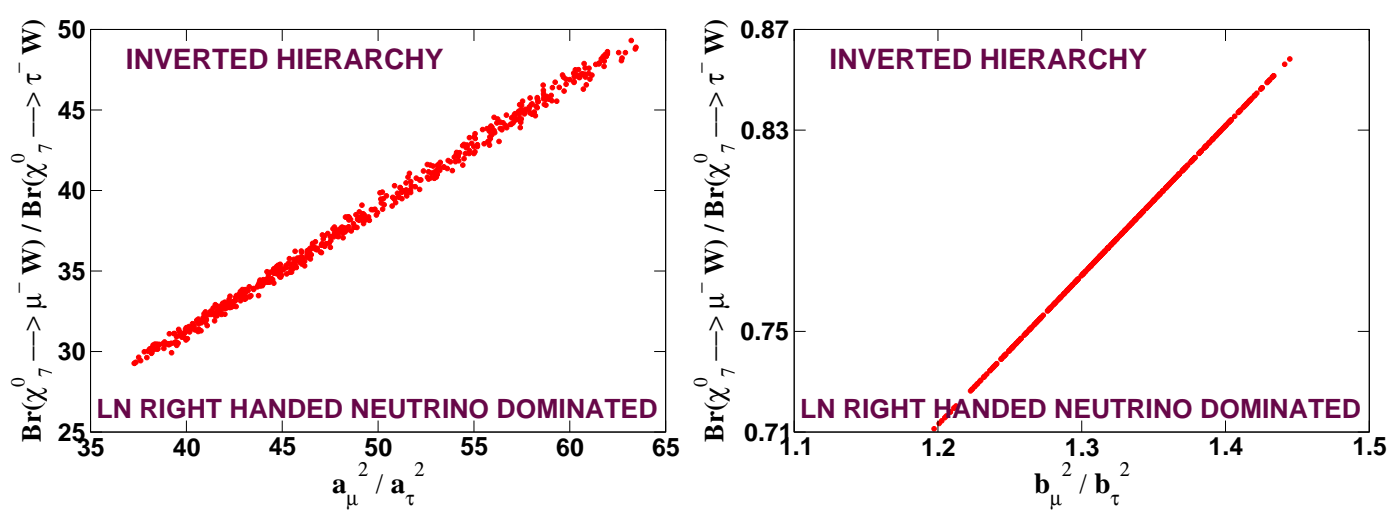

Figure 17: Ratio $\frac{B R\left(\tilde{\chi}_{7}^{0} \longrightarrow \mu W\right)}{B R\left(\tilde{\chi}_{7}^{0} \longrightarrow \tau W\right)}$ versus $\frac{a_{\mu}^{2}}{a_{\tau}^{2}}$ (left) and versus $\frac{b_{\mu}^{2}}{b_{\tau}^{2}}$ (right) plot for a $\nu^{c}$ like lightest neutralino $\left(\tilde{\chi}_{7}^{0}\right)$ with $\nu^{c}$ component $\left(\left|N_{75}\right|^{2}+\left|N_{76}\right|^{2}+\left|N_{77}\right|^{2}\right)>0.99$ (left), and $>0.97$ (right). Neutrino mass pattern is inverted hierarchical. Choice of parameters are for (left) $M_{1}=445 \mathrm{GeV}$, $\lambda=0.29, \kappa=0.07,\left(A_{\lambda} \lambda\right)=-8.2 \mathrm{TeV} \times \lambda,\left(A_{\kappa} \kappa\right)=165 \mathrm{GeV} \times \kappa, m_{\tilde{\nu}^{c}}=50 \mathrm{GeV}$ and $m_{\tilde{L}}=835 \mathrm{GeV}$ and for (right) $M_{1}=445 \mathrm{GeV}, \lambda=0.10, \kappa=0.07,\left(A_{\lambda} \lambda\right)=-2 \mathrm{TeV} \times \lambda,\left(A_{\kappa} \kappa\right)=165 \mathrm{GeV} \times$ $\kappa, m_{\tilde{\nu}^{c}}=50 \mathrm{GeV}$ and $m_{\tilde{L}}=835 \mathrm{GeV}$. Mass of the lightest neutralino is $129.4 \mathrm{GeV}$ (left) and 119.8 $\mathrm{GeV}$ (right) respectively.

With the inverted hierarchical neutrino mass pattern, in case (i) one observes a sharp correlation of the ratio $\frac{B R\left(\tilde{\chi}_{7}^{0} \longrightarrow \mu W\right)}{B R\left(\tilde{\chi}_{7}^{0} \longrightarrow \tau W\right)}$ with $\frac{a_{\mu}^{2}}{a_{\tau}^{2}}$ (see fig. 17). The other two ratios $\frac{B R\left(\tilde{\chi}_{7}^{0} \longrightarrow e W\right)}{B R\left(\tilde{\chi}_{7}^{0} \longrightarrow \mu W\right)}$ and $\frac{B R\left(\tilde{\chi}_{7}^{0} \longrightarrow e W\right)}{B R\left(\tilde{\chi}_{7}^{0} \longrightarrow \tau W\right)}$ do not show very sharp correlations with $\frac{a_{e}^{2}}{a_{\mu}^{2}}$ and $\frac{a_{e}^{2}}{a_{\tau}^{2}}$, respectively and we do not plot them here. However, in case (ii) all the three ratios show nice correlations with the corresponding $b_{i}^{2} / b_{j}^{2}$. We have shown this in fig.17 only for $b_{\mu}^{2} / b_{\tau}^{2}$. In this case the variations of the ratios of branching ratios with neutrino mixing angles are shown in fig. 18 .

For the case (i), only $\tan ^{2} \theta_{13}$ shows certain correlation with the ratio of branching ratio shown in fig.18 (right), but we do not show it here.

Finally, we would like to mention that in all these different cases discussed above, the lightest neutralino can have a finite decay length which can produce displaced vertices in the vertex detectors. Depending on the composition of the lightest neutralino, one can have different decay lengths. For example, a bino-dominated lightest neutralino can produce a displaced vertex $\sim$ a few $\mathrm{mm}$. Similarly, for a higgsino dominated lightest neutralino, decay vertices of the order of a few cms can be observed. On the other hand, if the lightest neutralino is $\nu^{c}$ dominated, then the decay lengths can be of the order of a few meters. These are very unique predictions of this model which can, in principle, be tested at the LHC.

\section{Summary and Conclusion}

In this work we have studied a supersymmetric model in detail where the observed pattern of neutrino mass-squared differences and mixing angles are obtained with the help of 


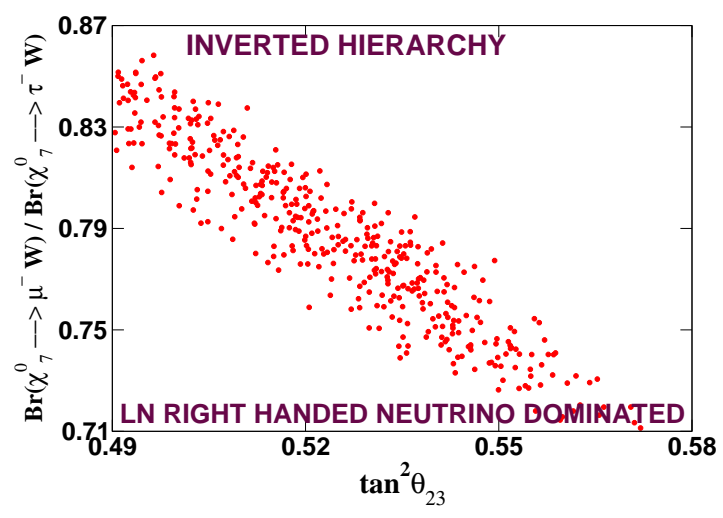

Figure 18: Ratio $\frac{B R\left(\tilde{\chi}_{7}^{0} \longrightarrow \mu W\right)}{B R\left(\tilde{\chi}_{7}^{0} \longrightarrow \tau W\right)}$ versus $\tan ^{2} \theta_{23} \quad$ (left), $\frac{B R\left(\tilde{\chi}_{7}^{0} \longrightarrow e W\right)}{\sqrt{B R\left(\tilde{\chi}_{7}^{0} \longrightarrow \mu W\right)^{2}+B R\left(\tilde{\chi}_{7}^{0} \longrightarrow \tau W\right)^{2}}}$ with $\tan ^{2} \theta_{12}$ (right) plot for a $\nu^{c}$ dominated lightest neutralino with $\nu^{c}$ component $\left(\left|N_{75}\right|^{2}+\left|N_{76}\right|^{2}+\right.$ $\left.\left|N_{77}\right|^{2}\right)>0.97$. Neutrino mass pattern is inverted hierarchical. Choice of parameters are same as that of fig. 17 .

three standard model gauge-singlet neutrino superfields, which simultaneously solve the $\mu$ problem of MSSM. The additional terms in the superpotential and the scalar potential include R-parity violating interactions involving these gauge-singlet neutrino superfields. The vacuum expectation values of the singlet sneutrinos give rise to effective Majorana mass terms for the singlet neutrinos, as well as a $\mu$-term, both at the electroweak scale. This model was introduced in ref. [8] and some phenomenology was discussed for a single gauge-singlet neutrino superfield. The spectrum and parameter space of this model, with three gauge singlet neutrino superfields, were discussed in [9]. We have performed a detailed and extensive analysis of this model in the neutrino and neutralino sector with the inclusion of three generation of gauge singlet neutrino superfields along with the associated interaction terms. The neutrino mass matrix is obtained because of the electroweak scale seesaw mechanism involving the gauge-singlet neutrinos and the mixing between the MSSM neutralinos and the neutrinos. We have done a thorough and systematic study of the neutrino mass matrix both analytically and numerically and tried to identify the relevant parameters which crucially control the bilarge pattern of neutrino mixing angles. We show that even with a flavour diagonal structure of the neutrino Yukawa coupling matrix, two large and one small mixing angles can be generated in this case. Both the normal and inverted hierarchical pattern of neutrino masses can be obtained with different hierarchies of the neutrino Yukawa couplings. Because of the presence of the neutrino-neutralino mixing, it is in general difficult to obtain a degenerate mass spectrum of the neutrinos and we do not consider this possibility in this paper.

We have also looked at the scalar sector of this model and wrote down the neutral scalar, pseudoscalar and charged scalar mass-squared matrices of this model. The allowed regions in the model parameters are obtained which satisfy certain constraints in the scalar sector. For example, absence of tachyons in the scalar squared-mass eigenvalues puts severe constraints in the relevant parameter space. With these choices of parameters, satisfying 
the scalar sector constraints, we have tried to fit the global three flavour neutrino data with both normal and inverted hierarchical mass spectrum of the neutrinos. Since this model involves several free parameters, we have made a few simplifying assumptions which makes this model more predictive.

Perhaps the most interesting part of this whole analysis is the study of the phenomenology of the lightest neutralino which can be the LSP in some cases or it can be the NLSP. The decay patterns of the lightest neutralino may provide additional information to find out more about the neutrino mass patterns and mixing angles. We have considered three different scenarios where the lightest neutralino can be either a bino-dominated one or a higgsino-dominated one or it can be mostly a gauge-singlet neutrino with very little mixing with the other states. We have also discussed briefly the production mechanism of the gauge-singlet neutrino dominated lightest neutralino at the LHC. The study of the decay pattern and the production mechanism of the lightest neutralino in this last mentioned scenario is extremely important because it will help in probing the mass scale and the properties of the gauge-singlet neutrinos at the LHC. The presence of the gauge-singlet neutrino dominated lightest neutralino can also distinguish this model from the usual bilinear R-parity violating model of generating neutrino masses and mixing. An important test of this model, as a supersymmetric solution to the observed neutrino mass patterns and mixing, can be performed by measuring the ratios of the decay branching ratios of the lightest neutralino in the final states involving a charged lepton and a W-boson. We have shown explicitly that these ratios of branching ratios have certain correlations with the neutrino mixing angles which depend on the nature of the LSP as well as on the pattern of the neutrino mass hierarchies considered. The study of the higgsino dominated LSP case is also very important because it can provide information about the $\mu$ parameter which is determined in terms of the vacuum expectation values of the gauge-singlet sneutrinos. Thus one may have information about the gauge-singlet neutrino mass scale and its coupling, from the decay pattern of the higgsino dominated LSP. Collider phenomenology of this model at the LHC is very rich both in the fermionic and the scalar sector. For example, pair produced lightest neutralino at the LHC give rise to the final states $\mu \mu W W, \tau \tau W W$ and $\mu \tau W W$ with a certain ratio for their production rates nicely correlated with $\tan ^{2} \theta_{23}$. These production rates also depend on the dominant component of the lightest neutralino as well as on the different hierarchical patterns of the three light neutrino masses. Another important testable prediction of this model is the measurement of displaced vertices in the decay of the lightest neutralino. This decay length can vary in the range of a few $\mathrm{mm}$ to $\sim 1$ meter depending on the nature of the lightest neutralino. We hope to come back to these issues in a future publication [27].

\section{Acknowledgments}

PG would like to thank the Council of Scientific and Industrial Research, Govt. of India for the financial support received as a Junior Research Fellow. We thank U. Chattopadhyay, D. Das, B. Mukhopadhyaya and S.K. Rai for many useful comments and suggestions. We would also like to thank K. Ray for a very useful suggestion during the developement of 
the code for numerical calculations.

\section{A. Scalar mass squared matrices}

In this appendix we present the details of various scalar mass squared matrices. For the convenience of the reader let us repeat that the scalar mass squared matrices are now $8 \times 8$, considering all three generations of sneutrinos (both doublet and singlet) and charged sleptons. These enhancements are essentially due to the mixing of neutral Higgs bosons with both the doublet and singlet sneutrinos and the mixing of charged Higgs with the charged sleptons.

\section{A.1 Neutral scalar mass squared matrices}

The decomposition of various neutral scalar fields in $\operatorname{real}(\mathcal{R})$ and imaginary $(\mathcal{I})$ parts are as follows

$$
\begin{aligned}
H_{1}^{0} & =H_{1 \mathcal{R}}^{0}+i H_{1 \mathcal{I}}^{0}, \\
H_{2}^{0} & =H_{2 \mathcal{R}}^{0}+i H_{2 \mathcal{I}}^{0}, \\
\tilde{\nu}_{k}^{c} & =\tilde{\nu}_{k \mathcal{R}}^{c}+i \tilde{\nu}_{k \mathcal{I}}^{c}, \\
\tilde{\nu}_{k} & =\tilde{\nu}_{k \mathcal{R}}+i \tilde{\nu}_{k \mathcal{I}} .
\end{aligned}
$$

Only the real components get VEVs as indicated in eq. 2.3.).

The entries of the scalar and pseudoscalar mass-squared matrices are defined as

$$
\begin{aligned}
\left(M_{P}^{2}\right)^{\alpha \beta} & =\left\langle\frac{1}{2} \frac{\partial^{2} V_{\text {neutral }}}{\partial \phi_{\mathcal{I}}^{\alpha} \partial \phi_{\mathcal{I}}^{\beta}}\right\rangle, \\
\left(M_{S}^{2}\right)^{\alpha \beta} & =\left\langle\frac{1}{2} \frac{\partial^{2} V_{\text {neutral }}}{\partial \phi_{\mathcal{R}}^{\alpha} \partial \phi_{\mathcal{R}}^{\beta}}\right\rangle,
\end{aligned}
$$

where

$$
\begin{aligned}
\phi_{\mathcal{I}}^{\alpha} & =H_{1 \mathcal{I}}^{0}, H_{2 \mathcal{I}}^{0}, \tilde{\nu}_{k \mathcal{I}}^{c}, \tilde{\nu}_{k \mathcal{I}}, \\
\phi_{\mathcal{R}}^{\alpha} & =H_{1 \mathcal{R}}^{0}, H_{2 \mathcal{R}}^{0}, \tilde{\nu}_{k \mathcal{R}}^{c}, \tilde{\nu}_{k \mathcal{R}} .
\end{aligned}
$$

Note that the Greek indices $\alpha, \beta$ are used to refer various scalar and pseudoscalar Higgs and

both doublet and singlet sneutrinos, that is $H_{1}^{0}, H_{2}^{0}, \tilde{\nu}_{k}^{c}, \tilde{\nu}_{k}$, whereas k is used as a subscript to specify various flavours of doublet and singlet sneutrinos i.e., $k=e, \mu, \tau$ in the flavour (weak interaction) basis.

\section{A.1.1 CP-odd neutral mass squared matrix}

The basis for CP-odd or pseudoscalar mass-squared matrix is

$$
\Phi_{P}^{T}=\left(H_{1 \mathcal{I}}^{0}, H_{2 \mathcal{I}}^{0}, \tilde{\nu}_{n \mathcal{I}}^{c}, \tilde{\nu}_{n \mathcal{I}}\right) \text {. }
$$


The pseudoscalar mass term in the Lagrangian is of the form

$$
\mathcal{L}_{\text {pseudoscalar }}^{\text {mass }}=\Phi_{P}^{T} \mathcal{M}_{P}^{2} \Phi_{P}
$$

where $\mathcal{M}_{P}^{2}$ is an $8 \times 8$ symmetric matrix. Using eq.(2.7), (2.8), and eq.(2.9), the independent elements are given by

$$
\begin{aligned}
& \left(M_{P}^{2}\right)^{H_{1 \mathcal{I}}^{0} H_{1 \mathcal{I}}^{0}}=\frac{1}{v_{1}}\left[\sum_{j} \lambda^{j} v_{2}\left(\sum_{i k} \kappa^{i j k} \nu_{i}^{c} \nu_{k}^{c}\right)+\sum_{j} \lambda^{j} r^{j} v_{2}^{2}+\mu \sum_{j} r_{c}^{j} \nu_{j}+\sum_{i}\left(A_{\lambda} \lambda\right)^{i} \nu_{i}^{c} v_{2}\right], \\
& \left(M_{P}^{2}\right)^{H_{1 \mathcal{I}}^{0} H_{2 \mathcal{I}}^{0}}=\sum_{i, j, k} \lambda^{j} \kappa^{i j k} \nu_{i}^{c} \nu_{k}^{c}+\sum_{i}\left(A_{\lambda} \lambda\right)^{i} \nu_{i}^{c}, \\
& \left(M_{P}^{2}\right)^{H_{2 \mathcal{I}}^{0} H_{2 \mathcal{I}}^{0}}=\frac{1}{v_{2}}\left[-\sum_{j} \rho^{j}\left(\sum_{l, k} \kappa^{l j k} \nu_{l}^{c} \nu_{k}^{c}\right)-\sum_{i, j}\left(A_{\nu} Y_{\nu}\right)^{i j} \nu_{i} \nu_{j}^{c}+\sum_{i}\left(A_{\lambda} \lambda\right)^{i} \nu_{i}^{c} v_{1}\right], \\
& \left(M_{P}^{2}\right)^{H_{1 \mathcal{L}}^{0} \tilde{\nu}_{m \mathcal{I}}^{c}}=-2 \sum_{j} \lambda^{j} u_{c}^{m j} v_{2}-\mu r^{m}+\lambda^{m} \sum_{i} r_{c}^{i} \nu_{i}+\left(A_{\lambda} \lambda\right)^{m} v_{2}, \\
& \left(M_{P}^{2}\right)^{H_{1 \mathcal{I}}^{0} \tilde{\nu}_{m \mathcal{I}}}=-\sum_{j} \lambda^{j} Y_{\nu}^{m j} v_{2}^{2}-\mu r_{c}^{m}, \\
& \left(M_{P}^{2}\right)^{H_{2 \mathcal{I}}^{0} \tilde{\nu}_{m \mathcal{I}}^{c}}=2 \sum_{j} u_{c}^{m j} \rho^{j}-\sum_{i}\left(A_{\nu} Y_{\nu}\right)^{i m} \nu_{i}+\left(A_{\lambda} \lambda\right)^{m} v_{1}, \\
& \left(M_{P}^{2}\right)^{H_{2 \mathcal{I}}^{0} \tilde{\nu}_{m \mathcal{I}}}=-\sum_{i, j, k} Y_{\nu}^{m j} \kappa^{i j k} \nu_{i}^{c} \nu_{k}^{c}-\sum_{j}\left(A_{\nu} Y_{\nu}\right)^{m j} \nu_{j}^{c}, \\
& \left(M_{P}^{2}\right)^{\tilde{\nu}_{n \mathcal{I}}^{c} \tilde{\nu}_{m \mathcal{I}}^{c}}=-2 \sum_{j} \kappa^{j n m} \zeta^{j}+4 \sum_{j} u_{c}^{m j} u_{c}^{n j}+\rho^{m} \rho^{n}+h^{n m} v_{2}^{2}+\left(m_{\tilde{\nu}^{c}}^{2}\right)^{n m}-2 \sum_{i}\left(A_{\kappa} \kappa\right)^{i n m} \nu_{i}^{c}, \\
& \left(M_{P}^{2} \tilde{\nu}_{n \mathcal{I}}^{c} \tilde{\nu}_{m \mathcal{I}}=2 \sum_{j} u_{c}^{m j} Y_{\nu}^{n j} v_{2}-Y_{\nu}^{n m} \sum_{i} r_{c}^{i} \nu_{i}+r_{c}^{n} r^{m}+\mu v_{1} Y_{\nu}^{n m}-\lambda^{m} r_{c}^{n} v_{1}-\left(A_{\nu} Y_{\nu}\right)^{n m} v_{2},\right. \\
& \left(M_{P}^{2}\right)^{\tilde{\nu}_{n \mathcal{I}} \tilde{\nu}_{m \mathcal{I}}}=\sum_{j} Y_{\nu}^{m j} Y_{\nu}^{n j} v_{2}^{2}+r_{c}^{m} r_{c}^{n}+\left(m_{\tilde{L}}^{2}\right)^{n m}+\gamma_{g} \xi_{v} \delta_{m n},
\end{aligned}
$$

where

$$
h^{n m}=\lambda^{n} \lambda^{m}+\sum_{i} Y_{\nu}^{i n} Y_{\nu}^{i m}
$$

We have checked that one eigenvalue of this $8 \times 8$ matrix is zero corresponding to the neutral Goldstone boson.

\section{A.1.2 CP-even neutral mass squared matrix}

The basis for the CP-even or scalar mass-squared matrix is

$$
\Phi_{S}^{T}=\left(H_{1 \mathcal{R}}^{0}, H_{2 \mathcal{R}}^{0}, \tilde{\nu}_{n \mathcal{R}}^{c}, \tilde{\nu}_{n \mathcal{R}}\right) .
$$

The scalar mass term in the Lagrangian is of the form 


$$
\mathcal{L}_{\text {scalar }}^{\text {mass }}=\Phi_{S}^{T} \mathcal{M}_{S}^{2} \Phi_{S}
$$

where $\mathcal{M}_{S}^{2}$ is an $8 \times 8$ symmetric matrix. The independent entries using eq.(2.7), (2.8), and eq.(2.9) are given by

$$
\begin{aligned}
& \left(M_{S}^{2}\right)^{H_{1 \mathcal{R}}^{0} H_{1 \mathcal{R}}^{0}}=\frac{1}{v_{1}}\left[\sum_{j} \lambda^{j} v_{2}\left(\sum_{i k} \kappa^{i j k} \nu_{i}^{c} \nu_{k}^{c}\right)+\sum_{j} \lambda^{j} r^{j} v_{2}^{2}+\mu \sum_{j} r_{c}^{j} \nu_{j}+\sum_{i}\left(A_{\lambda} \lambda\right)^{i} \nu_{i}^{c} v_{2}\right]+2 \gamma_{g} v_{1}^{2}, \\
& \left(M_{S}^{2}\right)^{H_{1 \mathcal{R}}^{0} H_{2 \mathcal{R}}^{0}}=-2 \sum_{j} \lambda^{j} \rho^{j} v_{2}-\sum_{i, j, k} \lambda^{j} \kappa^{i j k} \nu_{i} \nu_{k}^{c}-2 \gamma_{g} v_{1} v_{2}-\sum_{i}\left(A_{\lambda} \lambda\right)^{i} \nu_{i}^{c}, \\
& \left(M_{S}^{2}\right)^{H_{2 \mathcal{R}}^{0} H_{2 \mathcal{R}}^{0}}=\frac{1}{v_{2}}\left[-\sum_{j} \rho^{j}\left(\sum_{l, k} \kappa^{l j k} \nu_{l}^{c} \nu_{k}^{c}\right)-\sum_{i, j}\left(A_{\nu} Y_{\nu}\right)^{i j} \nu_{i} \nu_{j}^{c}+\sum_{i}\left(A_{\lambda} \lambda\right)^{i} \nu_{i}^{c} v_{1}\right]+2 \gamma_{g} v_{2}^{2}, \\
& \left(M_{S}^{2}\right)^{H_{1 \mathcal{R}}^{0} \tilde{\nu}_{m \mathcal{R}}^{c}}=-2 \sum_{j} \lambda^{j} u_{c}^{m j} v_{2}+2 \mu v_{1} \lambda^{m}-\lambda^{m} \sum_{i} r_{c}^{i} \nu_{i}-\mu r^{m}-\left(A_{\lambda} \lambda\right)^{m} v_{2}, \\
& \left(M_{S}^{2}\right)^{H_{1 \mathcal{R}}^{0} \tilde{\nu}_{m \mathcal{R}}}=-\sum_{j} \lambda^{j} Y_{\nu}^{m j} v_{2}^{2}-\mu r_{c}^{m}+2 \gamma_{g} \nu_{m} v_{1}, \\
& \left(M_{S}^{2}\right)^{H_{2 \mathcal{R}}^{0} \tilde{\nu}_{m \mathcal{R}}^{c}}=2 \sum_{j} u_{c}^{m j} \rho^{j}+2 \lambda^{m} \mu v_{2}+2 \sum_{i} Y_{\nu}^{i m} r_{c}^{i} v_{2}+\sum_{i}\left(A_{\nu} Y_{\nu}\right)^{i m} \nu_{i}-\left(A_{\lambda} \lambda\right)^{m} v_{1}, \\
& \left(M_{S}^{2}\right)^{H_{2 \mathcal{R}}^{0} \tilde{\nu}_{m \mathcal{R}}}=2 \sum_{j} Y_{\nu}^{m j} \rho^{j} v_{2}+\sum_{i, j, k} Y_{\nu}^{m j} \kappa^{i j k} \nu_{i}^{c} \nu_{k}^{c}-2 \gamma_{g} \nu_{m} v_{2}+\sum_{j}\left(A_{\nu} Y_{\nu}\right)^{m j} \nu_{j}^{c}, \\
& \left(M_{S}^{2}\right)^{\tilde{\nu}_{n \mathcal{R}}^{c} \tilde{\nu}_{m \mathcal{R}}^{c}}=2 \sum_{j} \kappa^{j n m} \zeta^{j}+4 \sum_{j} u_{c}^{m j} u_{c}^{n j}+\rho^{m} \rho^{n}+h^{n m} v_{2}^{2}+\left(m_{\tilde{\nu}^{c}}^{2}\right)^{m n}+2 \sum_{i}\left(A_{\kappa} \kappa\right)^{i m n} \nu_{i}^{c}, \\
& \left(M_{S}^{2}\right)^{\tilde{\nu}_{n \mathcal{R}}^{c} \tilde{\nu}_{m \mathcal{R}}}=2 \sum_{j} Y_{\nu}^{n j} u_{c}^{m j} v_{2}+Y_{\nu}^{n m} \sum_{i} r_{c}^{i} \nu_{i}+r_{c}^{n} r^{m}-\mu v_{1} Y_{\nu}^{n m}-\lambda^{m} r_{c}^{n} v_{1}+\left(A_{\nu} Y_{\nu}\right)^{n m} v_{2}, \\
& \left(M_{S}^{2}\right)^{\tilde{\nu}_{n \mathcal{R}} \tilde{\nu}_{m \mathcal{R}}}=\sum_{j} Y_{\nu}^{n j} Y_{\nu}^{m j} v_{2}^{2}+r_{c}^{m} r_{c}^{n}+\gamma_{g} \xi_{v} \delta_{n m}+2 \gamma_{g} \nu_{n} \nu_{m}+\left(m_{\tilde{L}}^{2}\right)^{m n},
\end{aligned}
$$

where eq.(A.7) has been used.

\section{A.2 Charged scalar mass squared matrix}

The charged scalar mass squared matrix considering all three generations of both left handed and right handed charged sleptons is also an $8 \times 8$ matrix. For the sake of completeness, here we give the expressions for various elements of the charged scalar mass-squared matrix. The elements of the charged scalar mass-squared matrix are defined as

$$
\left(M_{C}^{2}\right)^{\alpha \beta}=\left\langle\frac{1}{2} \frac{\partial^{2} V_{\text {charged }}}{\partial \phi^{c^{\alpha}} \partial \phi^{c^{\beta}}}\right\rangle
$$

where

$$
\phi^{c^{\alpha}}=H_{1}^{+}, H_{2}^{+}, \tilde{e}_{R k}^{+}, \tilde{e}_{L k}^{+} .
$$


The basis for charged scalar mass-squared matrix is

$$
\Phi_{C}^{+^{T}}=\left(H_{1}^{+}, H_{2}^{+}, \tilde{e}_{R n}^{+}, \tilde{e}_{L n}^{+}\right),
$$

and the charged scalar mass term in the Lagrangian is of the form

$$
\mathcal{L}_{\text {charged scalar }}^{\text {mass }}=\Phi_{C}^{-{ }^{T}} \mathcal{M}_{C}^{2} \Phi_{C}^{+}
$$

where $\mathcal{M}_{C}^{2}$ is an $8 \times 8$ symmetric matrix. The independent elements of $\mathcal{M}_{C}^{2}$ using eqs.(2.7), (2.8), and eq.(2.9) are given by

$$
\begin{aligned}
\left(M_{C}^{2}\right)^{H_{1} H_{1}} & =\frac{1}{v_{1}}\left[\sum_{j} \lambda^{j} \zeta^{j} v_{2}+\mu \sum_{j} r_{c}^{j} \nu_{j}+\sum_{i}\left(A_{\lambda} \lambda\right)^{i} \nu_{i}^{c} v_{2}\right]+\sum_{i, j, k} Y_{e}^{i j} Y_{e}^{k j} \nu_{i} \nu_{k}-\frac{g_{2}^{2}}{2}\left(\sum_{i} \nu_{i}^{2}-v_{2}^{2}\right) \\
\left(M_{C}^{2}\right)^{H_{1} H_{2}} & =-\sum_{j} \lambda^{j^{2}} v_{1} v_{2}+\sum_{j} \lambda^{j} r^{j} v_{2}+\sum_{j} \lambda^{j} u_{c}^{i j} \nu_{i}^{c}+\frac{g_{2}^{2}}{2} v_{1} v_{2}+\sum_{i}\left(A_{\lambda} \lambda\right)^{i} \nu_{i}^{c}, \\
\left(M_{C}^{2}\right)^{H_{2} H_{2}} & =\frac{1}{v_{2}}\left[-\sum_{j} \rho^{j} \zeta^{j}-\sum_{i, j}\left(A_{\nu} Y_{\nu}\right)^{i j} \nu_{i} \nu_{j}^{c}+\sum_{i}\left(A_{\lambda} \lambda\right)^{i} \nu_{i}^{c} v_{1}\right]+\frac{g_{2}^{2}}{2}\left(\sum_{i} \nu_{i}^{2}+v_{1}^{2}\right), \\
\left(M_{C}^{2}\right)^{H_{1} \tilde{e}_{R m}} & =-\sum_{i} r_{c}^{i} Y_{e}^{i m} v_{2}-\sum_{i}\left(A_{e} Y_{e}\right)^{i m} \nu_{i}, \\
\left(M_{C}^{2}\right)^{H_{1} \tilde{e}_{L m}} & =-\mu r_{c}^{m}-\sum_{i, j} Y_{e}^{m j} Y_{e}^{i j} \nu_{i} v_{1}+\frac{g_{2}^{2}}{2} \nu_{m} v_{1}, \\
\left(M_{C}^{2}\right)^{H_{2} \tilde{e}_{R m}} & =-\mu \sum_{i} Y_{e}^{m i} \nu_{i}-\sum_{i} Y_{e}^{i m} r_{c}^{i} v_{1}, \\
\left(M_{C}^{2}\right)^{H_{2} \tilde{e}_{L m}} & =-\sum_{j} Y_{\nu}^{m j} \zeta^{j}+\frac{g_{2}^{2}}{2} \nu_{m} v_{2}-\sum_{i}\left(A_{\nu} Y_{\nu}\right)^{m i} \nu_{i}^{c}, \\
\left(M_{C}^{2}\right)^{\tilde{e}_{R n} \tilde{e}_{R m}} & =\sum_{i, j} Y_{e}^{i m} Y_{e}^{j n} \nu_{i} \nu_{j}+\sum_{i} Y_{e}^{i m} Y_{e}^{i n} v_{1}^{2}+\left(m_{\tilde{e}^{c}}^{2}\right)^{m n}-\frac{g_{1}^{2}}{2} \xi_{v} \delta_{m n}, \\
\left(M_{C}^{2}\right)^{\tilde{e}_{R n} \tilde{e}_{L m}} & =-\mu Y_{e}^{m n} v_{2}+\left(A_{e} Y_{e}\right)^{n m} v_{1}, \\
\left(M_{C}^{2}\right)^{\tilde{e}_{L n} \tilde{e}_{L m}} & =r_{c}^{m} r_{c}^{n}+\sum_{j} Y_{e}^{m j} Y_{e}^{n j} v_{1}^{2}+\gamma_{g} \xi_{v} \delta_{m n}-\frac{g_{2}^{2}}{2} \xi_{v} \delta_{m n}+\frac{g_{2}^{2}}{2} \nu_{m} \nu_{n}+\left(m_{\tilde{L}}^{2}\right)^{m n} .
\end{aligned}
$$

As mentioned earlier, we have computed the eigenvalues of the charged scalar masssquared matrix numerically and ensured that seven of its eigenvalues are positive and there is a charged Goldstone boson.

\section{B. Feynman rules}

In this appendix we will study the relevant Feynman rules for the LSP decay calculations 28, 29. The required Feynman rules are

1. Neutralino-neutralino $-Z^{0}$,

2. Chargino-neutralino $-W^{ \pm}$. 


\section{B.1 Neutralino-neutralino- $Z^{0}$ and chargino-chargino- $Z^{0}, \gamma$}

For neutralinos the following relations between mass and weak eigenstates are very useful

$$
\begin{aligned}
& P_{L} \tilde{B}^{0}=P_{L} N_{i 1}^{*} \tilde{\chi}_{i}^{0}, \\
& P_{R} \tilde{B}^{0}=P_{R} N_{i 1} \tilde{\chi}_{i}^{0}, \\
& P_{L} \tilde{W}_{3}^{0}=P_{L} N_{i 2}^{*} \tilde{\chi}_{i}^{0}, \\
& P_{R} \tilde{W}_{3}^{0}=P_{R} N_{i 2} \tilde{\chi}_{i}^{0}, \\
& P_{L} \tilde{H}_{j}=P_{L} N_{i, j+2}^{*} \tilde{\chi}_{i}^{0}, \\
& P_{R} \tilde{H}_{j}=P_{R} N_{i, j+2} \tilde{\chi}_{i}^{0}, \quad \text { where } \quad j=1,2, \\
& P_{L} \nu_{L}^{k}=P_{L} N_{i, k+7}^{*} \tilde{\chi}_{i}^{0}, \\
& P_{R} \nu_{L}^{k}=P_{R} N_{i, k+7} \tilde{\chi}_{i}^{0}, \\
& P_{L} \nu_{R}^{c^{k}}=P_{L} N_{i, k+4}^{*} \tilde{\chi}_{i}^{0}, \\
& P_{R} \nu_{R}^{c^{k}}=P_{R} N_{i, k+4} \tilde{\chi}_{i}^{0}, \quad \text { where } \quad k=1,2,3,
\end{aligned}
$$

with i varies from 1 to 10 and

$$
P_{L}=\left(\frac{1-\gamma^{5}}{2}\right), \quad P_{R}=\left(\frac{1+\gamma^{5}}{2}\right) .
$$

In terms of the four component spinors $\chi_{i}$ for charginos, the following relations between mass and weak eigenstates are very useful

$$
\begin{aligned}
& P_{L} \tilde{W}=P_{L} V_{i 1}^{*} \tilde{\chi}_{i}, \\
& P_{R} \tilde{W}=P_{R} U_{i 1} \tilde{\chi}_{i}, \\
& P_{L} \tilde{H}=P_{L} V_{i 2}^{*} \tilde{\chi}_{i}, \\
& P_{R} \tilde{H}=P_{R} U_{i 2} \tilde{\chi}_{i}, \\
& P_{L} l^{j}=P_{L} V_{i, j+2}^{*} \tilde{\chi}_{i}, \\
& P_{R} l^{j}=P_{R} U_{i, j+2} \tilde{\chi}_{i},
\end{aligned}
$$

where $j=1,2,3$, and i varies from 1 to 5 .

So in terms of physical or mass eigenstates of charginos and neutralinos the required interaction terms are as follows

$$
\begin{aligned}
\mathcal{L}_{Z^{0} \tilde{\chi} \tilde{\chi}}= & \left(\frac{g}{\cos \theta_{W}}\right) Z_{\mu} \overline{\tilde{\chi}}_{i}^{+} \gamma^{\mu}\left[O_{i j}^{\prime L} P_{L}+O_{i j}^{\prime R} P_{R}\right] \tilde{\chi}_{j}^{+} \\
& +\left(\frac{g}{2 \cos \theta_{W}}\right) Z_{\mu} \overline{\tilde{\chi}}_{i}^{0} \gamma^{\mu}\left[O_{i j}^{\prime \prime} P_{L}+O_{i j}^{\prime \prime} P_{R}\right] \tilde{\chi}_{j}^{0}, \\
\mathcal{L}_{\gamma \tilde{\chi}^{+} \tilde{\chi}^{-}}= & -e A_{\mu} \overline{\tilde{\chi}}_{i}^{+} \gamma^{\mu} \tilde{\chi}_{i}^{+},
\end{aligned}
$$


where

$$
\begin{aligned}
O_{i j}^{\prime L} & =-V_{i 1} V_{j 1}^{*}-\frac{1}{2} V_{i 2} V_{j 2}^{*}+\delta_{i j} \sin ^{2} \theta_{W}, \\
O_{i j}^{\prime R} & =-U_{i 1}^{*} U_{j 1}-\frac{1}{2} U_{i 2}^{*} U_{j 2}-\frac{1}{2} U_{i, k+2}^{*} U_{j, k+2},+\delta_{i j} \sin ^{2} \theta_{W}, \\
O_{i j}^{\prime \prime^{L}} & =-\frac{1}{2} N_{i 3} N_{j 3}^{*}+\frac{1}{2} N_{i 4} N_{j 4}^{*}-\frac{1}{2} N_{i, k+7} N_{j, k+7}^{*}, \\
O_{i j}^{\prime \prime^{R}} & =-O_{i j}^{\prime \prime^{L}}, k=1,2,3 .
\end{aligned}
$$

In deriving eq. $(\mathrm{B.5})$ unitary properties of $\mathrm{U}$ and $\mathrm{V}$ matrices has been used.
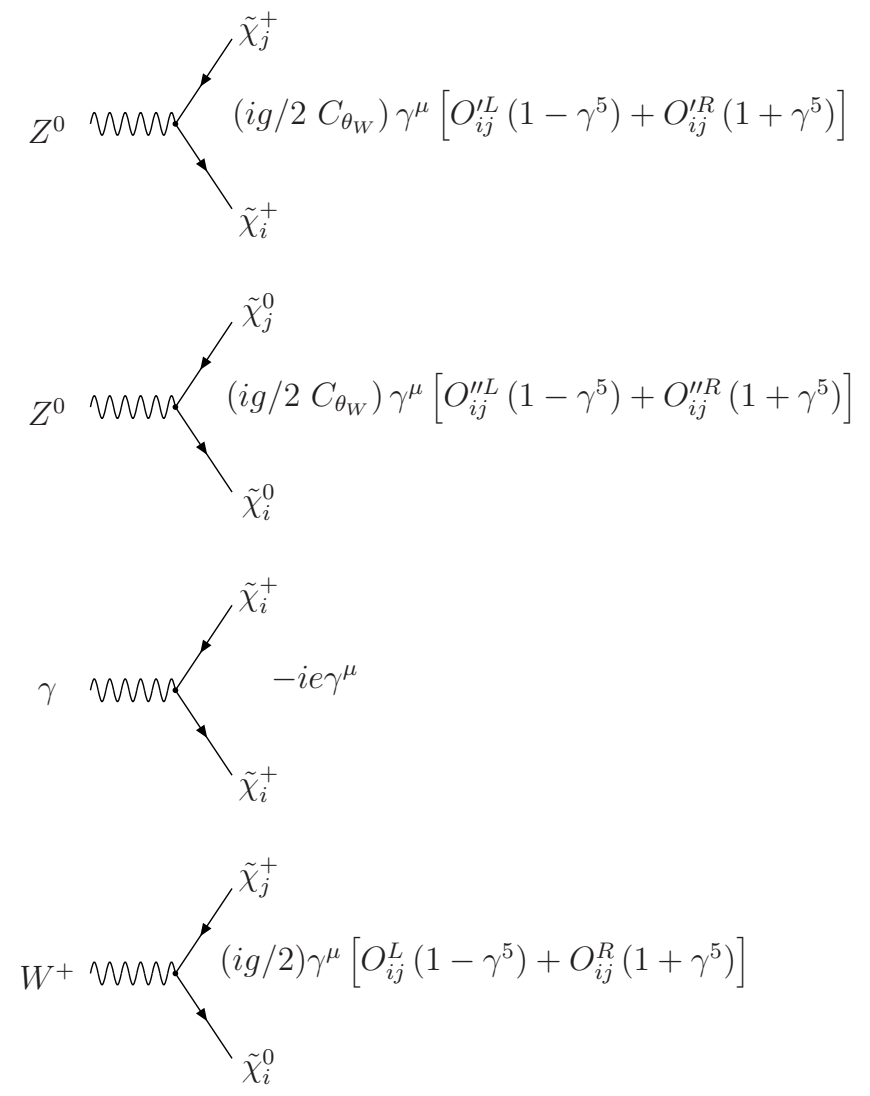

where $C_{\theta_{W}}=\cos \theta_{W}$

Figure 19: Feynman rules for interaction of neutral and charged gauge boson with charginos and neutralinos.

\section{B.2 Chargino-neutralino - $W^{ \pm}$}

Now in terms of physical chargino and neutralino states the relevant interaction term is

$$
\mathcal{L}_{W^{\mp} \tilde{\chi}^{ \pm} \tilde{\chi}^{0}}=\mathcal{L}_{W-\tilde{\chi}^{+} \tilde{\chi}^{0}}+\mathcal{L}_{W^{+} \tilde{\chi}^{-} \tilde{\chi}^{0}}
$$

where $\mathcal{L}_{W^{+}} \tilde{\chi}^{-} \tilde{\chi}^{0}$ is the hermitian conjugate of $\mathcal{L}_{W^{-}} \tilde{\chi}^{+} \tilde{\chi}^{0}$ and

$$
\mathcal{L}_{W^{-}} \tilde{\chi}^{+} \tilde{\chi}^{0}=g W_{\mu}^{-} \overline{\tilde{\chi}}_{i}^{0} \gamma^{\mu}\left[O_{i j}^{L} P_{L}+O_{i j}^{R} P_{R}\right] \tilde{\chi}_{j}^{+},
$$


with

$$
\begin{aligned}
& O_{i j}^{L}=N_{i 2} V_{j 1}^{*}-\frac{1}{\sqrt{2}} N_{i 4} V_{j 2}^{*}, \\
& O_{i j}^{R}=N_{i 2}^{*} U_{j 1}+\frac{1}{\sqrt{2}} N_{i 3}^{*} U_{j 2}+\frac{1}{\sqrt{2}} N_{i, k+7}^{*} U_{j, k+2},
\end{aligned}
$$

where $k=1,2,3$.

The Feynman rules are shown in fig.19. The matrices $O_{i j}^{\prime L}, O_{i j}^{\prime R}, O_{i j}^{\prime \prime L}, O_{i j}^{\prime \prime R}$ and $O_{i j}^{L}$, $O_{i j}^{R}$ are defined by eq.(B.5) and eq.(B.8), respectively.

\section{References}

[1] See,for example,B. Kayser, Neutrino Mass, Mixing, and Flavor Change, arXiv:0804.1497 [hep-ph] and references therein.

[2] T. Schwetz, M. Tortola and J. W. F. Valle, Three-flavour neutrino oscillation update, New. J. Phys. 10 (2008) 113011 [arXiv:0808.2016 [hep-ph]].

[3] P. Fayet, Supergauge Invariant Extension Of The Higgs Mechanism And A Model For The Electron And Its Neutrino, Nucl. Phys. B 90 (1975) 104;

Spontaneously Broken Supersymmetric Theories Of Weak, Electromagnetic And Strong Interactions, Phys. Lett. B 69 (1977) 489;

G. R. Farrar and P. Fayet, Phenomenology Of The Production, Decay, And Detection Of New Hadronic States Associated With Supersymmetry, Phys. Lett. B 76 (1978) 575.

[4] C. S. Aulakh and R. N. Mohapatra, Neutrino As The Supersymmetric Partner Of The Majoron, Phys. Lett. B 119 (1982) 136;

L. J. Hall and M. Suzuki, Explicit R-Parity Breaking In Supersymmetric Models, Nucl. Phys. B 231 (1984) 419;

I. H. Lee, Lepton Number Violation In Softly Broken Supersymmetry, Phys. Lett. B 138 (1984) 121;

I. H. Lee, Lepton Number Violation In Softly Broken Supersymmetry. 2, Nucl. Phys. B 246 (1984) 120;

G. G. Ross and J. W. F. Valle, Supersymmetric Models Without R-Parity, Phys. Lett. B 151 (1985) 375;

J. R. Ellis, G. Gelmini, C. Jarlskog, G. G. Ross and J. W. F. Valle, Phenomenology of Supersymmetry With Broken R-Parity, Phys. Lett. B 150 (1985) 142.

[5] See, for example, A. S. Joshipura and M. Nowakowski, 'Just so' oscillations in supersymmetric standard model, Phys. Rev. D 51 (1995) 2421] [hep-ph/9408224];

Leptonic CP violation in supersymmetric Standard Model, Phys. Rev. D 51 (1995) 5271 hep-ph/9403349;

M. Nowakowski and A. Pilaftsis, $W$ and $Z$ boson interactions in supersymmetric models with explicit R-parity violation, Nucl. Phys. B 461 (1996) 19 hep-ph/9508271;

F. Borzumati, Y. Grossman, E. Nardi and Y. Nir, Neutrino masses and mixing in supersymmetric models without $R$ parity, Phys. Lett. B 384 (1996) 123 hep-ph/9606251;

S. Roy and B. Mukhopadhyaya, Some implications of a supersymmetric model with R-parity breaking bilinear interactions, Phys. Rev. D 55 (1997) 7020 hep-ph/9612447; 
K. Choi, K. Hwang and E. J. Chun, Atmospheric and solar neutrino masses from horizontal U(1) symmetry, Phys. Rev. D 60 (1999) 031301 hep-ph/9811363;

A. S. Joshipura and S. K. Vempati, Sneutrino vacuum expectation values and neutrino anomalies through trilinear R-parity violation, Phys. Rev. D 60 (1999) 111303

hep-ph/9903435;

E. J. Chun, S. K. Kang, C. W. Kim and U. W. Lee, Supersymmetric neutrino masses and mixing with R-parity violation, Nucl. Phys. B 544 (1999) 89 hep-ph/9807327;

A. Datta, B. Mukhopadhyaya and S. Roy, Constraining an R-parity violating supersymmetric theory from the SuperKamiokande data on atmospheric neutrinos, Phys. Rev. D 61 (2000) 055006 hep-ph/9905549;

M. Hirsch, M. A. Diaz, W. Porod, J. C. Romao and J. W. F. Valle, Neutrino masses and mixings from supersymmetry with bilinear R-parity violation: A theory for solar and atmospheric neutrino oscillations, Phys. Rev. D 62 (2000) 113008 [hep-ph/0004115]; Erratum-ibid. D 65 (2002) 119901;

M. A. Diaz, M. Hirsch, W. Porod, J. C. Romao and J. W. F. Valle, Solar neutrino masses and mixing from bilinear R-parity broken supersymmetry: Analytical versus numerical results, Phys. Rev. D 68 (2003) 013009 hep-ph/0302021;

Erratum-ibid. D 71 (2005) 059904;

F. Takayama and M. Yamaguchi, Pattern of neutrino oscillations in supersymmetry with bilinear R-parity violation, Phys. Lett. B 476 (2000) 116 hep-ph/9910320];

M. Hirsch, H. V. Klapdor-Kleingrothaus and S. G. Kovalenko, B-L violating masses in softly broken supersymmetry, Phys. Lett. B 398 (1997) 311 hep-ph/9701253;

Y. Grossman and H. E. Haber, Sneutrino mixing phenomena, Phys. Rev. Lett. 78 (1997) 3438 hep-ph/9702421;

(S)neutrino properties in R-parity violating supersymmetry. I: CP-conserving phenomena, Phys. Rev. D 59 (1999) 093008 hep-ph/9810536;

Y. Grossman and S. Rakshit, Neutrino masses in R-parity violating supersymmetric models, Phys. Rev. D 69 (2004) 093002 hep-ph/0311310.

[6] For reviews on R-parity violation, see, e.g., R. Barbier et al., R-parity violating supersymmetry, Phys. Rept. 420 (2005) 1 hep-ph/0406039;

M. Chemtob, Phenomenological constraints on broken $R$ parity symmetry in supersymmetry models, Prog. Part. Nucl. Phys. 54 (2005) 71 hep-ph/0406029.

[7] J. E. Kim and H. P. Nilles, The Mu Problem And The Strong CP Problem, Phys. Lett. B 138 (1984) 150.

[8] D. E. Lopez-Fogliani and C. Munoz, Proposal for a new minimal supersymmetric standard model, Phys. Rev. Lett. 97 (2006) 041801 hep-ph/0508297].

[9] N. Escudero, D. E. Lopez-Fogliani, C. Munoz and R. R. de Austri, Analysis of the parameter space and spectrum of the $\mu \nu S S M$, J. High Energy Phys. 0812 (2008) 099 arXiv:0810.1507 [hep-ph].

[10] R. Kitano and K. y. Oda, Neutrino masses in the supersymmetric standard model with right-handed neutrinos and spontaneous R-parity violation, Phys. Rev. D 61 (2000) 113001 hep-ph/9911327.

[11] P. N. Pandita and P. F. Paulraj, Infra-red stable fixed points of Yukawa couplings in non-minimal supersymmetric standard model with R-parity violation, Phys. Lett. B 462 (1999) 294 hep-ph/9907561; 
P. N. Pandita, Nonminimal supersymmetric standard model with baryon and lepton number violation, Phys. Rev. D 64 (2001) 056002 hep-ph/0103005;

M. Chemtob and P. N. Pandita, Nonminimal supersymmetric standard model with lepton number violation, Phys. Rev. D 73 (2006) 055012 [hep-ph/0601159];

A. Abada and G. Moreau, An origin for small neutrino masses in the NMSSM, J. High Energy Phys. 08 (2006) 044 hep-ph/0604216.

[12] A. Abada, G. Bhattacharyya and G. Moreau, A new mechanism of neutrino mass generation in the NMSSM with broken lepton number, Phys. Lett. B 642 (2006) 503 hep-ph/0606179].

[13] J. R. Ellis, J. F. Gunion, H. E. Haber, L. Roszkowski and F. Zwirner, Higgs Bosons in a Nonminimal Supersymmetric Model, Phys. Rev. D 39 (1989) 844.

[14] B. Mukhopadhyaya and R. Srikanth, Bilarge neutrino mixing in R-parity violating supersymmetry: The role of right-chiral neutrino superfields, Phys. Rev. D $\mathbf{7 4}$ (2006) 075001 hep-ph/0605109.

[15] Y. Farzan and J. W. F. Valle, R-parity violation assisted thermal leptogenesis in the seesaw mechanism, Phys. Rev. Lett. 96 (2006) 011601 hep-ph/0509280.

[16] J. R. Ellis, K. Enqvist, D. V. Nanopoulos, K. A. Olive, M. Quiros and F. Zwirner, Problems for (2,0) compactifications, Phys. Lett. B 176 (1986) 403 ;

B. Rai and G. Senjanovic, Gravity and domain wall problem, Phys. Rev. D 49 (1994) 2729 hep-ph/9301240;

S. A. Abel, S. Sarkar and P. L. White, On the Cosmological Domain Wall Problem for the Minimally Extended Supersymmetric Standard Model, Nucl. Phys. B 454 (1995) 663 hep-ph/9506359.

[17] S. A. Abel, Destabilising divergences in the NMSSM, Nucl. Phys. B 480 (1996) 55 hep-ph/9609323;

C. Panagiotakopoulos and K. Tamvakis, Stabilized NMSSM without domain walls, Phys. Lett. B 446 (1999) 224 hep-ph/9809475.

[18] Stephen Wolfram, The Mathematica Book, 5th ed. (Wolfram media, 2003).

[19] S. Eidelman et al. [Particle Data Group], Review of particle physics, Phys. Lett. B 592 (2004) 1 .

[20] S. Fukuda et al. [Super-Kamiokande Collaboration], Tau neutrinos favoured over sterile neutrinos in atmospheric muon neutrino oscillations, Phys. Rev. Lett. 85 (2000) 3999 hep-ex/0009001;

M. Ambrosio et al. [MACRO Collaboration], Matter effects in upward-going muons and sterile neutrino oscillations, Phys. Lett. B 517 (2001) 59 [hep-ex/0106049;

Q. R. Ahmad et al. [SNO Collaboration], Direct evidence for neutrino flavour transformation from neutral-current interactions in the Sudbury Neutrino Observatory, Phys. Rev. Lett. 89 (2002) 011301 nucl-ex/0204008;

Measurement of day and night neutrino energy spectra at SNO and constraints on neutrino mixing parameters, Phys. Rev. Lett. 89 (2002) 011302 hucl-ex/0204009;

S. N. Ahmed et al. [SNO Collaboration], Measurement of the total active B-8 solar neutrino flux at the Sudbury Neutrino Observatory with enhanced neutral current sensitivity, Phys. Rev. Lett. 92 (2004) 181301 nucl-ex/0309004.

[21] M. Apollonio et al. [CHOOZ Collaboration], Limits on Neutrino Oscillations from the CHOOZ Experiment, Phys. Lett. B 466 (1999) 415 hep-ex/9907037; 
K. Eguchi et al. [KamLAND Collaboration], First results from KamLAND: Evidence for reactor anti-neutrino disappearance, Phys. Rev. Lett. 90 (2003) 021802 hep-ex/0212021;

A. Bandyopadhyay, S. Choubey, S. Goswami, S. T. Petcov and D. P. Roy, Constraints on neutrino oscillation parameters from the SNO salt phase data, Phys. Lett. B 583 (2004) 134 hep-ph/0309174;

G. L. Fogli, E. Lisi, A. Marrone, D. Montanino, A. Palazzo and A. M. Rotunno, Addendum to: Solar neutrino oscillation parameters after first KamLAND results, Phys. Rev. D 69 (2004) 017301 hep-ph/0308055;

P. C. de Holanda and A. Y. Smirnov, Solar neutrinos: The SNO salt phase results and physics of conversion, Astropart. Phys. 21 (2004) 287 hep-ph/0309299;

M. Maltoni, T. Schwetz, M. A. Tortola and J. W. F. Valle, Status of global fits to neutrino oscillations, New. J. Phys. 6 (2004) 122 hep-ph/0405172;

A. Strumia and F. Vissani, Implications of neutrino data circa 2005, Nucl. Phys. B $\mathbf{7 2 6}$ (2005) 294 hep-ph/0503246.

[22] P. F. Harrison, D. H. Perkins and W. G. Scott, Tri-bimaximal mixing and the neutrino oscillation data, Phys. Lett. B 530 (2002) 167 [hep-ph/0202074].

[23] J. F. Gunion and H. E. Haber, Two-body decays of neutralinos and charginos, Phys. Rev. D 37 (1988) 2515.

[24] F. Franke and H. Fraas, Neutralinos and Higgs Bosons in the Next-To-Minimal Supersymmetric Standard Model, Int. J. Mod. Phys. A 12 (1997) 479 hep-ph/9512366;

Production and decay of neutralinos in the next-to-minimal supersymmetric standard model, Z. Phys. C 72 (1996) 309 hep-ph/9511275.

[25] B. Mukhopadhyaya, S. Roy and F. Vissani, Correlation between neutrino oscillations and collider signals of supersymmetry in an R-parity violating model, Phys. Lett. B 443 (1998) 191 hep-ph/9808265;

E. J. Chun and J. S. Lee, Implication of Super-Kamiokande data on R-parity violation, Phys. Rev. D 60 (1999) 075006 hep-ph/9811201;

S. Y. Choi, E. J. Chun, S. K. Kang and J. S. Lee, Neutrino oscillations and R-parity violating collider signals, Phys. Rev. D 60 (1999) 075002 hep-ph/9903465;

J. C. Romao, M. A. Diaz, M. Hirsch, W. Porod and J. W. F. Valle, A supersymmetric solution to the solar and atmospheric neutrino problems, Phys. Rev. D 61 (2000) 071703 hep-ph/9907499;

W. Porod, M. Hirsch, J. Romao and J. W. F. Valle, Testing neutrino mixing at future collider experiments, Phys. Rev. D 63 (2001) 115004 hep-ph/0011248.

[26] M. Hirsch, A. Vicente and W. Porod, Spontaneous R-parity violation: Lightest neutralino decays and neutrino mixing angles at future colliders, Phys. Rev. D 77 (2008) 075005 arXiv:0802.2896.

[27] P. Ghosh and S. Roy, Signatures of $\mu \nu S S M$ at the LHC, work in progress.

[28] H. E. Haber and G. L. Kane, The Search For Supersymmetry: Probing Physics Beyond The Standard Model, Phys. Rept. 117 (1985) 75.

[29] J. F. Gunion and H. E. Haber, Higgs Bosons In Supersymmetric Models. 1, Nucl. Phys. B 272 (1986) 1;

Erratum-ibid. B 402 (1993) 567;

J. F. Gunion and H. E. Haber, Higgs Bosons in Supersymmetric Models. 2. Implications for Phenomenology, Nucl. Phys. B 278 (1986) 449. 